\title{
Irrigated Croplands, Estimated Pumpage, and Water-Level Changes in Diamond Valley, Eureka and Elko Counties, Nevada, through 1990
}

By FREDDY E. ARTEAGA, J. LARUE SMITH, and JAMES R. HARRILL

U.S. GEOLOGICAL SURVEY

Open-File Report 95-107

Prepared in cooperation with the NEVADA DIVISION OF WATER RESOURCES

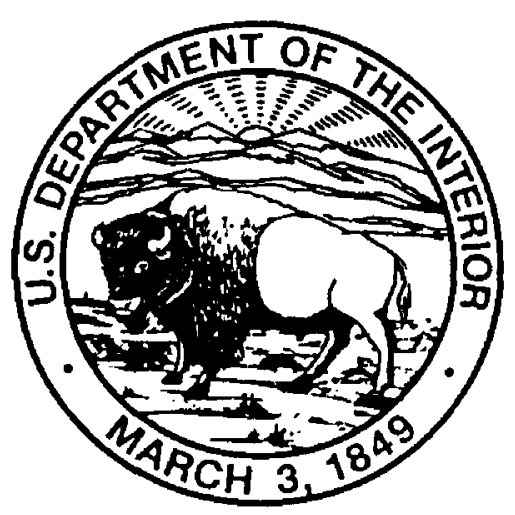

Carson City, Nevada 1995 


\title{
U.S. DEPARTMENT OF THE INTERIOR BRUCE BABBITT, Secretary
}

\author{
U.S. GEOLOGICAL SURVEY \\ GORDON P. EATON, Director
}

Any use of trade names in this publication is for descriptive purposes only and does not constitute endorsement by the U.S. Government.

For additional information write to:

District Chief

U.S. Geological Survey

333 West Nye Lane, Room 203

Carson City, NV 89706-0866
Copies of this report can be purchased from:

U.S. Geological Survey Earth Science Information Center Open-File Reports Section Box 25286, MS 517 Denver Federal Center Denver, CO 80225-0046 


\section{CONTENTS}

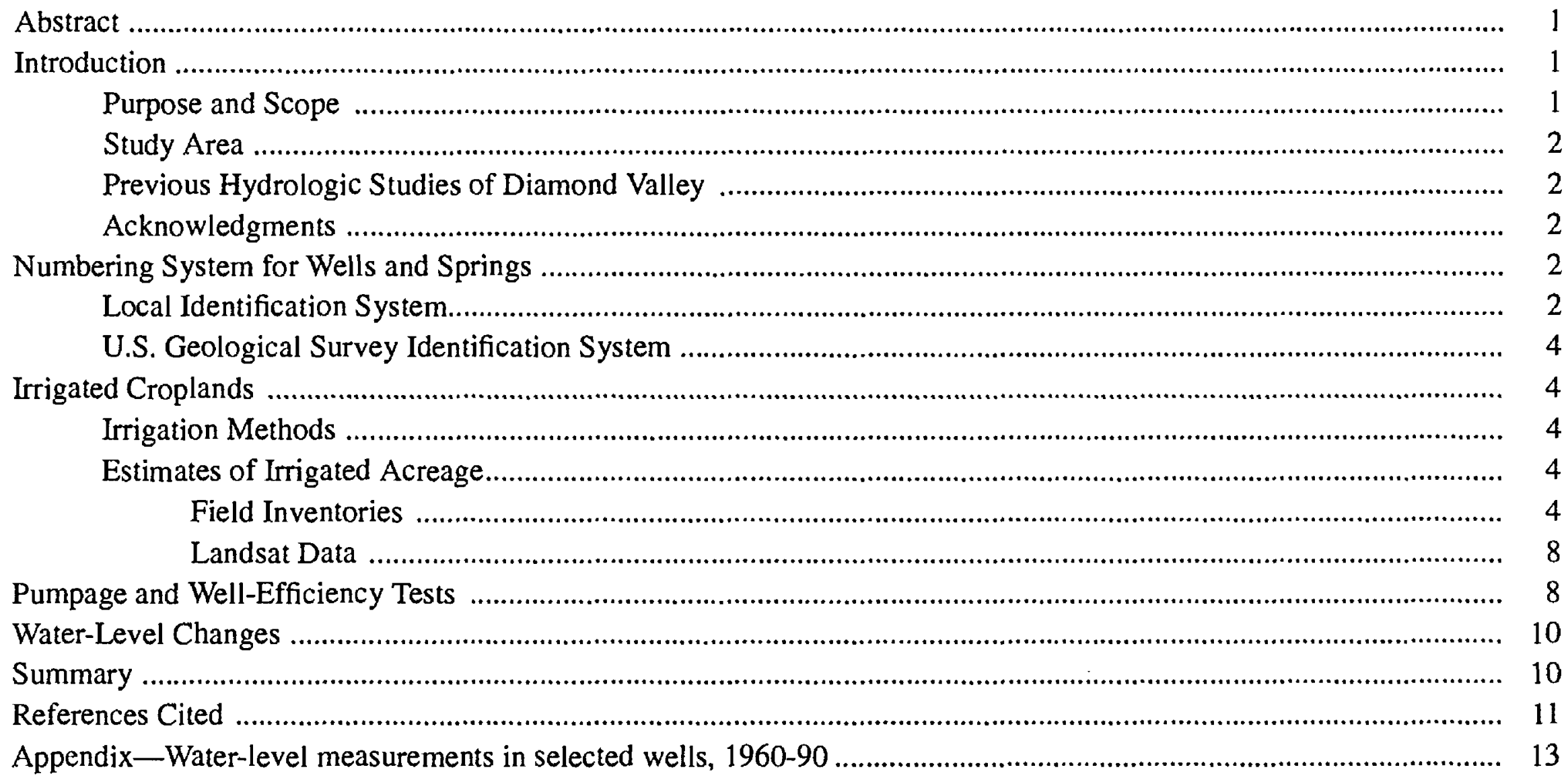

\section{PLATE}

1. Map showing irrigated croplands from Landsat image, estimated pumpage, water levels at selected wells, and flows at selected springs, Diamond Valley, Eureka County, Nevada

\section{FIGURES}

1. Map showing Diamond Valley hydrographic area and boundary between northern and southern Diamond Valley subareas

2-3. Graphs showing:

2. Reflectance spectra representing various land covers

3. Estimated crop acreage and estimated pumpage in Diamond Valley, 1950-90

4. Boxplots showing results of well-efficiency tests made between 1975-89 for discharge, pumping level, total head, power consumed to pump 1 acre-foot of water, and normalized power consumed to lift 1 acre-foot of water 1 foot.

\section{TABLE}

1. Estimated irrigated acreage and estimated pumpage in Diamond Valley, Nev., 1950-90 


\section{CONVERSION FACTORS, VERTICAL DATUM, AND OTHER ABBREVIATIONS USED IN REPORT}

\begin{tabular}{rll}
\hline Multiply & By & To obtain \\
\hline acre & 4,047 & square meter \\
acre-foot (acre-ft) & 1,233 & cubic meter \\
acre-foot per year (acre-ft/yr) & 1,233 & cubic meter per year \\
foot (ft) & 0.3048 & meter \\
mile (mi) & 1.609 & kilometer \\
\hline
\end{tabular}

Temperature: Degrees Celsius $\left({ }^{\circ} \mathrm{C}\right)$ can be converted to degrees Fahrenheit $\left({ }^{\circ} \mathrm{F}\right)$ by using the formula ${ }^{\circ} \mathrm{F}=\left[1.8\left({ }^{\circ} \mathrm{C}\right)\right]+32$. Degrees Fahrenheit can be converted to degrees Celsius by using the formula ${ }^{\circ} \mathrm{C}=0.556\left({ }^{\circ} \mathrm{F}-32\right)$.

Sea level: In this report, "sea level" refers to the National Geodetic Vertical Datum of 1929 (NGVD of 1929, formerly called "Sea-Level Datum of 1929"), which is derived from a general adjustment of the first-order leveling networks of the United States and Canada.

Other abbreviations:

kilowatthour

kilowatthour per acre-foot kilowatthour per acre-foot, per foot
$\mathrm{kWh}$

$\mathrm{kWh} / \mathrm{acre}-\mathrm{ft}$

(kWh/acre-ft)/ft 


\title{
Irrigated Croplands, Estimated Pumpage, and Water-Level Changes in Diamond Valley, Eureka and Elko Counties, Nevada, through 1990
}

\author{
By Freddy E. Arteaga, J. LaRue Smith, and James R. Harrill
}

\section{ABSTRACT}

Ground-water pumpage and crop data were compiled as part of an investigation of ground-water withdrawals in Diamond Valley, Eureka and Elko Counties, Nevada. Cumulative ground-water withdrawals from 1950 to 1990 exceeded 1.2 million acre-feet-an average of more than 29,000 acre-feet per year. During 1972-90, pumpage of ground water for irrigation increased from about 23,000 acre-feet to about 64,000 acre-feet. The net decline in ground-water levels has been about 50 feet in the developed area of southern Diamond Valley. Field investigations during the summer of 1990 indicated that of 291 irrigation wells visited, 158 were in operation. Landsat imagery and a geographical information system were used to determine that about 19,400 acres of land were irrigated in 1989. The area of croplands irrigated by a typical centerpivot sprinkler in 1989 (determined from the Landsat image) was about 125 acres compared to the 135 acres that can potentially be irrigated on a 160 -acre field by that system. The use of 125 acres as the area irrigated by a typical centerpivot sprinkler to calculate total irrigated acreage might improve the accuracy of estimates derived from field inventories made throughout the State.

\section{INTRODUCTION}

Diamond Valley has supported pumping for irrigation since 1949 when two wells were drilled on the eastern side of the valley to develop land under the
Desert Land Act (Eakin, 1962, p. 5). Development continued at a moderate rate through the 1950's and 1960 's. By 1965, more than 200 irrigation wells had been drilled; however, probably not more than 80 had been pumped in any single growing season (Harrill, 1968 , p. 6). In 1972, electric power became available, and the number of wells pumped for irrigation increased markedly. The annual rate of pumping increased accordingly. In 1972, annual pumpage for irrigation was about 23,000 acre-ft. By 1975, estimates of annual pumpage exceeded the estimates of perennial yield. Large-scale pumping for irrigation continued successfully through 1990 , and in 1990 , the amount of pumpage was about 64,000 acre-ft. Cumulative ground-water withdrawals from 1950 through 1990 exceeded 1.2 million acre-ft-an average of slightly more than 29,000 acre-ft/yr. In 1990, however, pumping was concentrated in the southern part of the valley and the overdraft was basinwide-annual pumpage continues to exceed the estimated perennial yield. Consequently, concerns exist about the long-term continuation of the development.

In 1990, the U.S. Geological Survey, in cooperation with the Nevada Division of Water Resources, began to compile information to better estimate pumpage from irrigation wells in Diamond Valley. The purpose of this effort was to provide the initial information needed to manage the groundwater resource.

\section{Purpose and Scope}

This report summarizes (1) irrigated acreage and pumpage reported by previous investigations (Eakin, 1962; and Harrill, 1968); (2) field inventories made by the Nevada Division of Water Resources from 
1966 through 1989; (3) remotely sensed Landsat Thematic Mapper (TM) data recorded July 30, 1989, that show the extent of irrigated land; (4) well-efficiency tests made by the University of Nevada Cooperative Extension Service, Eureka County; and (5) water-level measurements made by the Nevada Division of Water Resources and U.S. Geological Survey through 1990. The investigation also included a field inventory of 291 wells in 1990, evaluation of consumptive use by crops, and determination of power-consumption coefficients from results of well-efficiency tests.

\section{Study Area}

Diamond Valley is an intermontane valley in east-central Nevada. Most of the valley, including all the farmlands, is in Eureka County; a small part of the valley extends into Elko County (fig. 1). Eureka, the county seat of Eureka County, is in the southern part of the valley and is the only town in the study area. The population of the county, estimated at 767 in 1960 (Nevada State Department of Administration, 1990), had increased to 2,110 by 1990 (Maud Naroll, Office of the Nevada State Demographer, Bureau of Business and Economic Records, University of Nevada, Reno, written commun., 1991). Much of this population growth is because of a surge of mining activity in the area. In a previous study of Diamond Valley, Harrill (1968) divided the area into two subareas: southern and northern Diamond Valley (fig. 1). Southern Diamond Valley, about 276,000 acres, is where the field canvas of wells was done and where the 158 irrigation wells were in use. In comparison, northern Diamond Valley, about 194,000 acres, contains fewer than 10 irrigation wells, and no field work was done there.

\section{Previous Hydrologic Studies of Diamond Valley}

Eakin (1962) completed a preliminary evaluation of the hydrology of Diamond Valley. Harrill (1968) provided a moderately detailed appraisal of water supply in the valley, including a ground-water budget, chemical analyses of water, estimates of irrigated acreage and pumpage, and estimates of consumptive use for major crops. Pennington (1980) evaluated evapotranspiration rates for alfalfa in the valley during 1977-78.

\section{Acknowledgments}

The authors are grateful for the cooperation of the local residents of the valley in providing data and permitting access to their wells during this investigation. The authors also acknowledge the assistance received from Federal, State, and local governmental agencies. Locations of most of the wells and documentation of corresponding electric utility and meter numbers were checked in the field by James V. Enloe, Nevada Division of Water Resources. Data on wellefficiency tests were made available by Steve R. Lewis, Eureka County Cooperative Extension Service, University of Nevada, Reno.

\section{NUMBERING SYSTEMS FOR WELLS AND SPRINGS}

\section{Local Identification System}

A local site designation is used in Nevada to identify a site by the hydrographic area (Rush, 1968) and by the official rectangular subdivision of the public lands referenced to the Mount Diablo base line and meridian. Each site designation consists of four units: (1) the number of the hydrographic area; (2) the township, preceded by an $\mathrm{N}$ or $\mathrm{S}$ to indicate location north or south of the base line (half townships are indicated by an $\mathrm{H}$ following the township number); (3) the range, preceded by an $E$ to indicate location east of the meridian; and (4) the section number and letters designating the quarter section, quarter-quarter section, and so on (A, B, C, and D indicate the northeast, northwest, southwest, and southeast quarters, respectively), followed by a number indicating the sequence in which the site was recorded. For example, site 153 N20 E53 01BDDA1 is in Diamond Valley (hydrographic area 153). It is the first site recorded in the northeast quarter of the southeast quarter of the southeast quarter of the northwest quarter of section 1, Township 20 North, Range 53 East, Mount Diablo base line and meridian. 


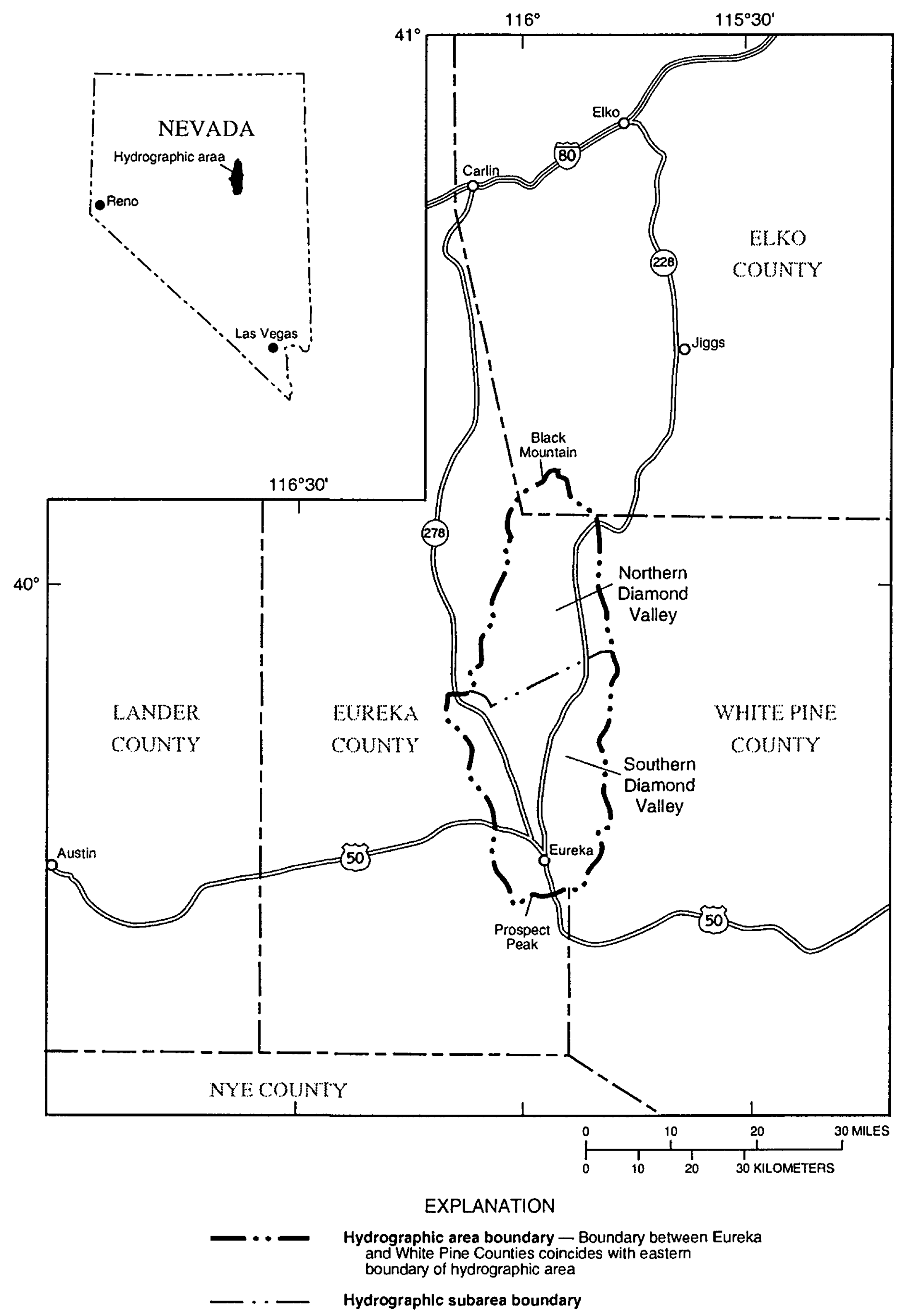

Figure 1. Location of Diamond Valley hydrographic area and boundary between northern and southern Diamond Valley hydrographic subareas. 


\section{Standard Identification System}

The U.S. Geological Survey identification (ID) system is based on latitude and longitude. The site ID indicates the geographic location of each site and provides a unique number for each. The ID consists of 15 digits: the first six digits denote the degrees, minutes, and seconds of latitude; the next seven digits denote degrees, minutes, and seconds of longitude; and the last two digits (assigned sequentially) identify the sites within a 1 -second grid. For example, site 393731115570301 is at $39^{\circ} 37^{\prime} 31^{\prime \prime}$ latitude and $115^{\circ} 57^{\prime} 03^{\prime \prime}$ longitude, and it is the first site recorded in that 1 -second grid. The originally assigned number is retained as a permanent identifier even if a more accurate latitude and longitude are later determined.

\section{IRRIGATED CROPLANDS}

\section{Irrigation Methods}

The three principal irrigation methods used in Diamond Valley are center-pivot sprinkler systems, border irrigation, and furrow irrigation. In 1990, center-pivot, self-propelled sprinkler systemsthe primary form of irrigation in Diamond Valleywere used for approximately 16,000 acres. A single center-pivot system can cover a circular area of about 135 acres (U.S. Soil Conservation Service, 1981, p. NV 685-10). Border irrigation and flood irrigation were used for the remaining area under cultivationapproximately 6,000 acres.

\section{Estimates of Irrigated Acreage}

\section{Field Inventories}

Field inventories of irrigated acreage have been made by the Nevada Division of Water Resources during 1966-69 and 1975-89 and by the U.S. Geological Survey in 1990 . These inventories, along with previously published information on ground-water pumpage (Harrill, 1968, p. 48-49), are listed in table 1.

\section{Landsat Data}

The field inventories reported in table 1 are labor intensive, and detailed inventories involve committing considerable time and resources. Another technique being used with increasing frequency is remote sensing. Remote sensing has been used extensively for large inventories of irrigated acreage, especially in the arid and semi-arid regions of the United States. For example, Heimes and Luckey (1983) used remote sensing to inventory irrigation in the High Plains in parts of Colorado, Kansas, Nebraska, New Mexico, Oklahoma, South Dakota, Texas, and Wyoming. The U.S. Geological Survey has incorporated remotely sensed Landsat data and geographic information system (GIS) technology into a method to identify irrigated acreage, and this method was applied in Diamond Valley. The satellite image chosen for analysis consisted of Landsat Thematic Mapper (TM) data (scene-identification number Y5197717534X0) from July 30, 1989.

The Landsat TM scanner measures radiation, or energy reflected by objects, at different wavelengths of the electromagnetic spectrum. Common land covers have distinctly different spectral patterns in the visible and near-infrared regions of the electromagnetic spectrum, as illustrated in figure 2. Irrigated croplands (represented by corn leaves in fig. 2) show a characteristic low reflectance in TM channel 3 and high reflectance in TM channel 4; playas have a high reflectance in all TM channels; and sage generally has a low reflectance in TM channels 1 through 3 , as well as a lower reflectance than irrigated croplands in TM channel 4. Reflectance from croplands contrasts with that of most native vegetation in Nevada.

Digital TM data can be processed to distinguish croplands from other land covers. Digital data from the satellite are stored as an array of cells, or pixels, as one data layer for each TM channel. Each pixel represents a square 30 -meter-by-30-meter area. Land cover is identified from the TM data by using the spectral pattern recognition technique known as unsupervised classification (Lillesand and Kiefer, 1987). Distinct spectral patterns are identified from the data, statistics about the patterns are tabulated, and each pattern is assigned a value. The data for each pixel in each layer are then compared to the spectral patterns. The value assigned to the pixel that the data most closely resemble is placed in a new, single data layer. The spectral patterns are analyzed and related to the appropriate land-cover type or class. This process is referred to as "land-cover classification." 
Table 1. Estimated irrigated acreage and estimated pumpage in Diamond Valley, Nevada, 1950-90

[Data for 1950-65 from Harrill (1968, tables 16 and 17, p. 48, 49); data for 1966-69 and 1975-89 from files of Nevada Division of Water Resources, Carson City; data for 1990 based on field inventories by U.S. Geological Survey; --, data not available]

\begin{tabular}{|c|c|c|c|c|c|c|c|c|}
\hline \multirow{2}{*}{ Year } & \multirow{2}{*}{$\begin{array}{l}\text { Number of } \\
\text { wells visited }\end{array}$} & \multirow{2}{*}{$\begin{array}{l}\text { Number of } \\
\text { active wells }\end{array}$} & \multicolumn{4}{|c|}{ Irrigated cropland (acres) } & \multirow{2}{*}{$\begin{array}{l}\text { Total Irrigated } \\
\text { acreage }\end{array}$} & \multirow{2}{*}{$\begin{array}{l}\text { Pumpage } \\
\text { (acre-feet }\end{array}$} \\
\hline & & & Alfalfa & Grain & Pasture & Other & & \\
\hline 1950 & - & -. & -- & -- & -- & -- & -- & 300 \\
\hline 1951 & -- & -- & -. & -. & .- & -- & -- & 600 \\
\hline 1952 & -- & -- & -- & .- & -- & -- & -- & 800 \\
\hline 1953 & -- & -- & -- & -- & -. & -- & -. & 800 \\
\hline 1954 & -- & -- & -- & -- & -- & -- & -- & 800 \\
\hline 1955 & .- & .- & -- & -- & -- & -- & -- & 1,000 \\
\hline 1956 & -. & -. & -- & -- & -- & -- & -- & 1,000 \\
\hline 1957 & -- & -- & -- & .- & -. & -- & -- & 1,200 \\
\hline 1958 & -. & -- & .- & .- & -- & -- & -- & 1,200 \\
\hline 1959 & -- & -- & -. & -- & -- & -- & -- & 1,800 \\
\hline 1960 & -- & .- & -- & -. & -- & -- & -- & 2,400 \\
\hline 1961 & -- & -- & 70 & 2,900 & -- & 220 & 3,200 & 6,100 \\
\hline 1962 & -- & -- & 300 & 3,000 & -- & 2,300 & 5,600 & 11,000 \\
\hline 1963 & .- & .- & 400 & 3,740 & -- & 700 & 4,800 & 9,700 \\
\hline 1964 & -- & -- & 985 & 4,710 & -- & 41 & 5,740 & 12,000 \\
\hline 1965 & 281 & $70-75$ & 2,130 & 5,450 & -- & 19 & 7,600 & 16,000 \\
\hline 1966 & -- & 74 & - & -- & -- & -- & 13,000 & 22,400 \\
\hline 1967 & -- & -- & -- & -- & -- & -- & 9,500 & 19,400 \\
\hline 1968 & -- & -- & -- & -- & -- & -- & 9,000 & 18,200 \\
\hline 1969 & -- & -- & - & -- & -- & -- & -- & 22,900 \\
\hline 1970-74: & \multicolumn{8}{|c|}{ No data available } \\
\hline 1975 & 228 & 125 & 10,700 & 5,250 & 0 & 1,860 & 17,800 & 53,400 \\
\hline 1976 & 232 & 137 & 10,800 & 6,760 & 0 & 1,190 & 18,700 & 56,200 \\
\hline 1977 & 233 & 143 & 12,800 & 6,650 & 515 & 0 & 19,800 & 53,000 \\
\hline 1978 & 233 & 164 & 14,800 & 5,800 & 0 & 1,260 & 21,900 & 59,800 \\
\hline 1979 & 234 & 172 & 15,800 & 5,910 & 0 & 830 & 22,600 & 61,800 \\
\hline 1980 & 240 & 187 & 17,400 & 5,130 & 0 & 545 & 23,100 & 64,000 \\
\hline 1981 & 238 & 183 & 20,700 & 4,090 & 0 & 485 & 25,300 & 71,700 \\
\hline 1982 & 242 & 180 & 22,300 & 2,580 & 0 & 445 & 25,300 & 73,300 \\
\hline 1983 & 243 & 188 & 22,000 & 2,580 & 0 & 240 & 24,800 & 71,900 \\
\hline 1984 & 248 & 189 & 24,300 & 2,400 & 0 & 100 & 26,800 & 78,100 \\
\hline 1985 & 248 & 189 & 24,300 & 2,400 & 0 & 100 & 26,800 & 78,100 \\
\hline 1986 & 249 & 174 & 21,100 & 2,860 & 126 & 100 & 24,200 & 69,600 \\
\hline 1987 & 249 & 166 & 20,100 & 2,210 & 660 & 0 & 23,000 & 66,000 \\
\hline 1988 & 249 & 163 & 20,200 & 1,060 & 0 & 295 & 21,600 & 63,400 \\
\hline 1989 & 239 & 168 & 19,800 & 2,660 & 0 & 1,070 & 23,500 & 66,700 \\
\hline 1990 & 291 & 158 & 19,900 & 2,010 & 150 & 135 & 22,200 & 64,400 \\
\hline
\end{tabular}



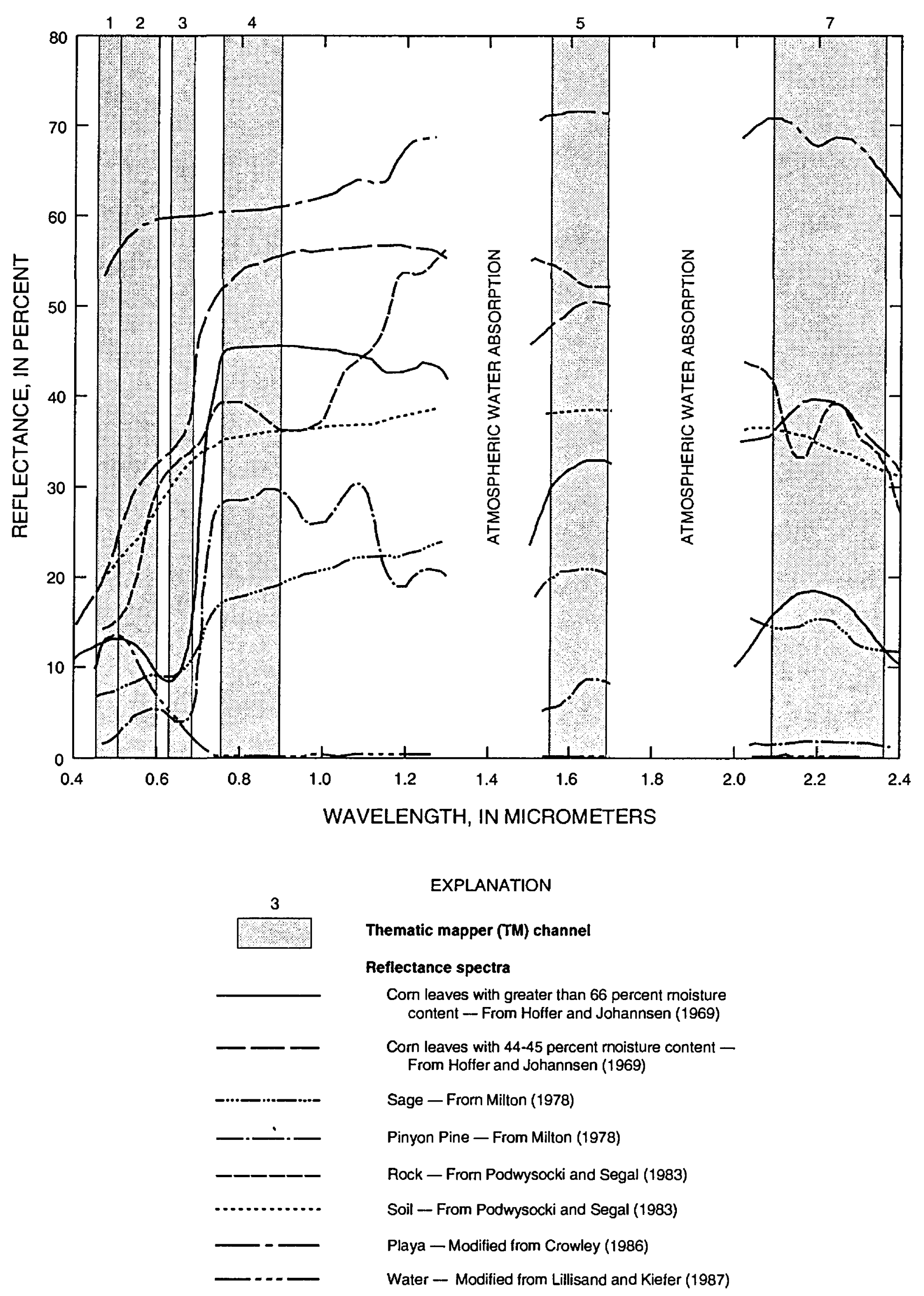

Figure 2. Spectra of various land covers show distinctly different reflectance characteristics. These differences are used in classifying land covers from satellite imagery. (Data are not from Diamond Valley.) 
The digital data are incorporated in a GIS for further analysis. After the classes of croplands are converted from image format to vector (line-based) format, they can be integrated with other digital-data layers, such as planimetric base features (roads, hydrography, and public-land survey system). Because the Landsat image and the GIS data layer for the land-cover classification of croplands are registered to each other, the two can be visually compared to check for errors or inconsistencies and improve the classification. For example, riparian vegetation (green vegetation along streams or areas of runoff from irrigated fields) may be spectrally inseparable from irrigated croplands and, therefore, be incorrectly classified as irrigated croplands. Also, irrigated areas where crops have recently been harvested may not show the reflectance characteristic of irrigated croplands and, therefore, may be incorrectly omitted from the classification. However, if analysts view the Landsat image with the land-cover classification, they can identify the characteristic stream or irrigation channel with riparian vegetation or the characteristic circular or rectangular shape of irrigated croplands and thereby refine the classification, edit the linework, and update the GIS. The interpretation of some areas of the image may not be as straightforward and may require more extensive fieldwork. An effective use of the remote sensing and GIS method is to (1) acquire a satellite TM scene at the peak of the growing season, (2) process the data for the land-cover classification immediately thereafter, and (3) provide maps for field verification and to assist in the field inventory for that growing season.

In this study, three classes of irrigated croplands were identified from the image of July 30,1989 : (1) irrigated croplands; (2) probably irrigated croplands; and (3) previously irrigated croplands (that is, farmlands that may have been taken out of production). Criteria used in the interpretation and refinement of the land-cover classification are as follows:

- Riparian areas that were easily recognized were removed from the classification.

- Areas that were spectrally classified as irrigated croplands and had well-defined circular or rectangular shapes, indicative of irrigation systems, were classified as irrigated.

- Areas that were spectrally classified as irrigated croplands but were not circular, rectangular, or riparian were classified as probably irrigated. These areas require field verification.

- Areas that appeared on the image to have been productive farmlands (circular or rectangular areas that have spectral features similar to that of irrigated croplands) but were not spectrally classified as irrigated croplands were reclassified as previously irrigated. These areas also require field verification.

Plate I shows the final classification. The areas calculated from the GIS are as follows:

- Class 1 includes 17,400 acres of irrigated croplands.

- Class 2 includes 2,000 acres of probably irrigated croplands.

- Class 3 includes 400 acres of previously irrigated croplands.

The total area of Class 1 and Class 2 lands19,400 acres - is 4,100 acres less than the estimate of total acreage in 1989 from the well inventories listed in table 1.

There are three probable reasons for the difference in total areas determined by field inventory and by image classification. First, the areas determined from the image represent irrigated croplands as of the end of July 1989. Crops harvested earlier and new crops planted later in the season were not included. Second, as determined from the Landsat data, the area of croplands irrigated by a single centerpivot sprinkler system on an 160-acre field in 1989 was typically about 125 acres. This number compares closely with the area of a circle with a radius of $0.25 \mathrm{mi}$, which is 125.6 acres. This area is smaller than the 135 acres that can potentially be irrigated by such a system. If 135 acres was used as the standard area irrigated by this system to calculate acreage, there would be a 10-acre difference between the acreage obtained by that calculation and that seen on an image. With 130 pivot systems active in 1989, this discrepancy accounts for about 1,300 acres of difference. Third, uneven water distribution or soil conditions may result in the green vegetation within a field being less than the total area of the irrigated field. Field checking of the images would be the best way to resolve these differences and would increase the accuracy of the assessment. 


\section{PUMPAGE AND WELL-EFFICIENCY TESTS}

Pumpage prior to 1966 was estimated by Harrill (1968, p. 49). Pumpage from 1966 to 1969 and from 1975 to 1989 was estimated by the Nevada Division of Water Resources, primarily on the basis of inventories of irrigated land and water duties (estimates are not available for 1970-74). These estimates are listed in table 1 and shown in figure 3, along with the estimates of acreage of irrigated croplands.

The University of Nevada Cooperative Extension Service office in Eureka, Nev., provided reports of 418 well-efficiency tests for Diamond Valley spanning 1972-89. The test reports contain information on approximate well location, type of pump and motor, water level, pumping level, friction head, pressure head, discharge rate, date and method of measurement, and overall efficiency. Complete information is available for 285 of the tests, and for these tests, additional computations were made to determine the power, in kilowatthours, needed to pump 1 acre-ft of water. A second variable was computed by dividing the kilowatthours per acre-foot by the total head (the sum of depth to pumping level, friction head, and pressure head) to determine the power consumed to lift 1 acre-ft of water $1 \mathrm{ft}$. Of the 285 tests with complete data, about 17 percent were in T. $20 \mathrm{~N}$.; about 71 percent were in $\mathrm{T} .21 \mathrm{~N}$.; about 11 percent were in T. $22 \mathrm{~N}$.; and about 1 percent were in $\mathrm{T} .23 \mathrm{~N}$. Most of the tests were made from the mid-1970's to the early 1980's; testing continued at a reduced rate through 1989. Graphs known as boxplots (Tukey, 1977) are used to display information on the statistical distribution of discharge, pumping level, total head, power consumed pumping each acre-foot of water, and power consumed lifting each acre-foot of water by $1 \mathrm{ft}$ (fig. 4) for three areas in the valley:

- Area 1 includes 49 tests from T. 20 N., R. 53 and $54 \mathrm{E}$;

- Area 2 includes 201 tests from T. 21 N., R. 53 and $54 \mathrm{E} . ;$ and

- Area 3 includes 32 tests from T. 22 N., R. 53 and $54 \mathrm{E}$.

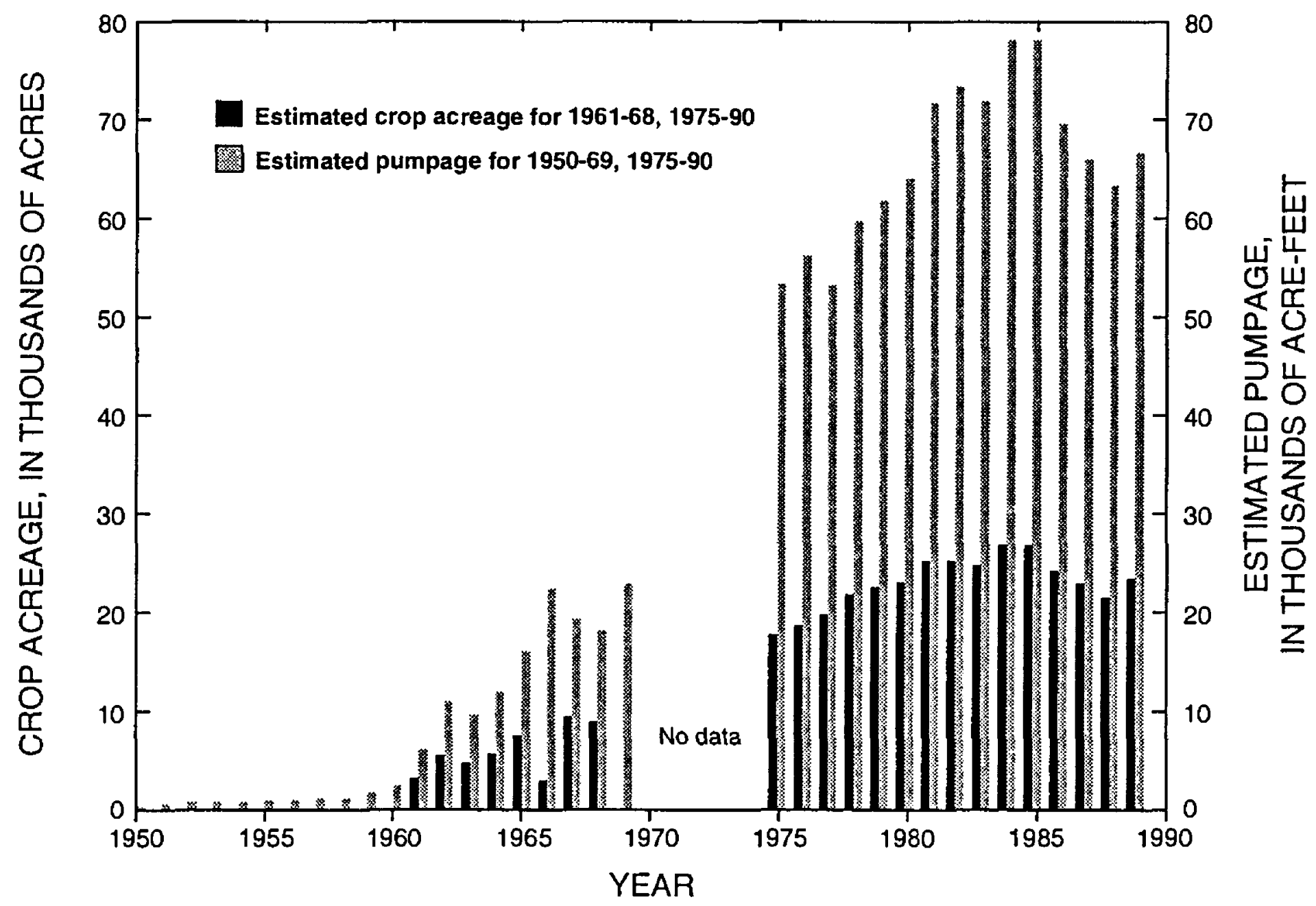

Figure 3. Estimated crop acreage and estimated pumpage in Diamond Valley, Nev., 1950-90. 

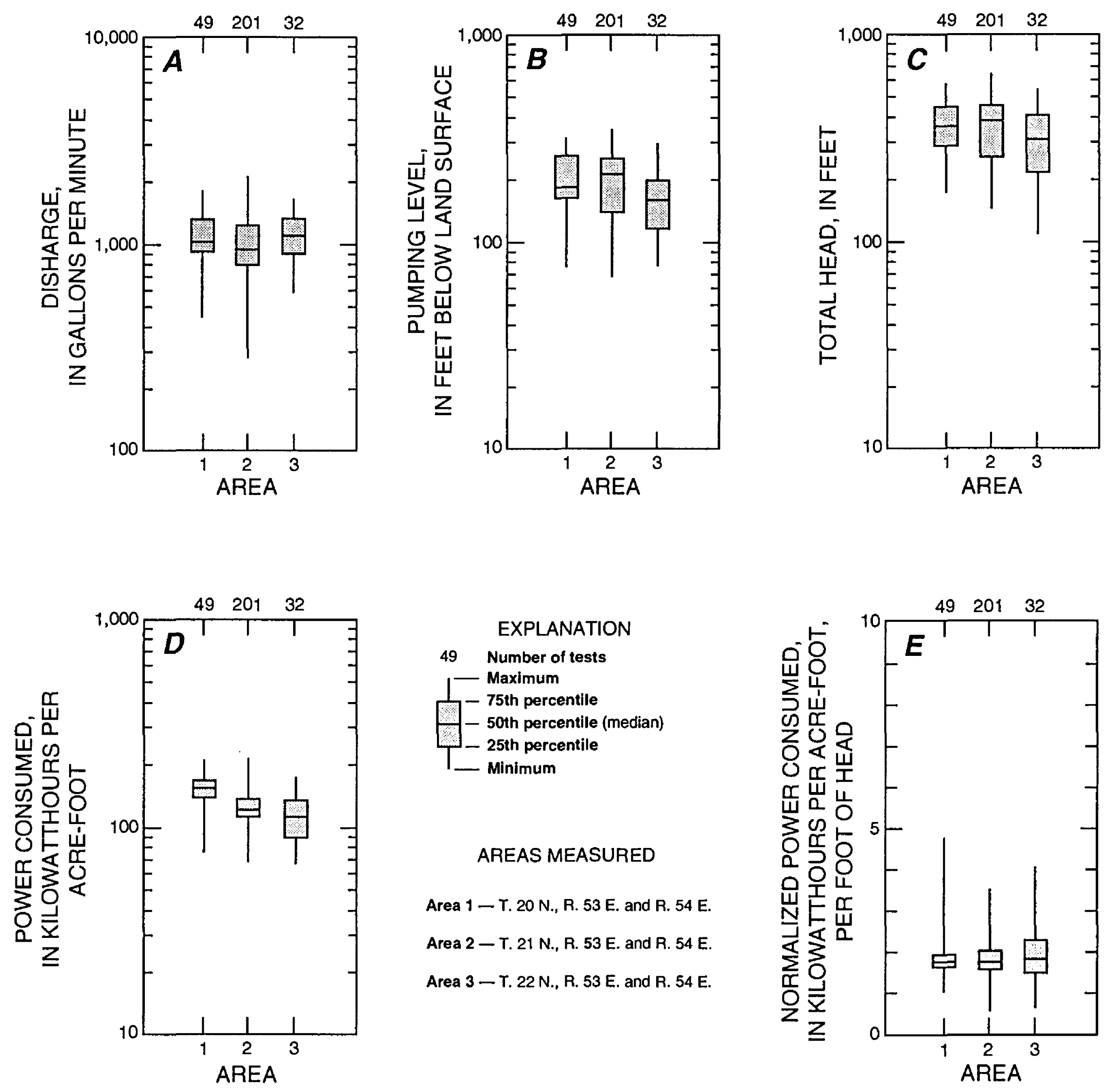

Figure 4. Boxplots showing results of well-efficiency tests made between 1975-89: $A$, discharge; $B$, pumping level; $C$, total head; $D$, power consumed to pump 1 acre-foot of water; and $E$, normalized power consumed to lift 1 acre-foot of water 1 foot. 
The number of tests and median values for each of the five variables are represented visually by features known as "boxes" and "whiskers" (fig. 4). A "box" defines the spread of the middle 50 percent of the data (the values that lie between the 25th and 75th percentiles). The median value of the data (the 50th percentile) is indicated by a horizontal line within the box. The vertical lines beyond each end of the box are called whiskers. They show the range for values that extend beyond the ends of the box to the maximum and minimum data values. The median values of power consumed per acre-foot of water, per foot of total head, are similar to the value $(1.8 \mathrm{kWh} /$ acre$\mathrm{ft} / \mathrm{ft}$ )-reported in a study of 60 wells in Idaho (Moreland, 1977, p. 16). Where adequate information on total head is available for specific irrigation seasons, pumpage can be roughly estimated from powerconsumption records by the following relation (from Moreland, 1977, fig. 4E):

$$
\text { Acre-feet pumped }=k W h / 1.8 T H,
$$

where $k W h$ is power used, in kilowatthours;

TH is total head, in feet; and

\section{8 is typical value of $k W h$ per acre-foot per foot of total head.}

The values of total head shown in figure $4 C$ span 1972-89. During that period, water levels declined throughout the pumping area (pl. 1), which would systematically decrease total head with time. However, during the same period, most irrigators converted from high-pressure to low-pressure sprinkler systems that are more efficient and, as a result, cause a increase in total head. Consequently, the total heads shown in figure $4 C$ should not be used to make estimates of pumpage for any specific year. If an independent check is desired on estimates that are based on crop inventories and duties of water, the preceding relation, supported by field determinations of total head, could be used to develop an independent estimate of pumpage.

\section{WATER-LEVEL CHANGES}

Water levels in most wells in the developed area of southern Diamond Valley have undergone a net decline of approximately $50 \mathrm{ft}$ since pumping for irrigation began. Water levels measured in selected wells over relatively long periods are listed in the appendix.
Hydrographs of 13 selected wells (pl. 1) show waterlevel changes during various periods. The rate of decline appears to have increased somewhat during the mid-1970's throughout the developed area. As of 1990, measured water levels in this area were declining at rates of 1.5 to $2.5 \mathrm{ft} / \mathrm{yr}$. Hydrographs of miscellaneous measurements at two springs, Shipley Hot Spring and Thompson Ranch Spring, are also shown on plate 1. Measurements of flow at Thompson Ranch Spring ranged from a high of about $1,900 \mathrm{gal} / \mathrm{min}$ in 1983 to $50 \mathrm{gal} / \mathrm{min}$ in 1990 .

\section{SUMMARY}

Ground-water pumpage and crop data were compiled as part of an investigation of ground-water withdrawals in Diamond Valley, Eureka and Elko Counties, Nev. Cumulative ground-water withdrawals from 1950 to 1990 exceeded 1.2 million acre-ft. The pumpage of ground water for irrigation increased from about 23,000 acre-ft in 1972 to about 64,000 acre-ft in 1990. The net decline in ground-water levels has been about 50 feet in the developed area of southern Diamond Valley.

Field investigations during the summer of 1990 indicated that of 291 irrigation wells visited, 158 were in operation. Landsat imagery and a geographical information system were used to determine that about 19,400 acres of croplands were irrigated in 1989. Also, the area of croplands irrigated by a typical center-pivot sprinkler in 1989 (determined from the Landsat image) was about 125 acres, compared to 135 acres that can potentially be irrigated on a 160 -acre field by a center-pivot system. The area of a circle with a radius of $0.25 \mathrm{mi}$ is 125.6 acres, which agrees closely with the areas of irrigated fields as determined from the Landsat image. The use of 125 acres as the area irrigated by a standard-sized pivot system to calculate total irrigated acreage might improve the accuracy of estimates derived from field inventories made throughout the State.

Well-efficiency tests are available for 418 wells in the valley. For these tests, the median power consumption required to lift 1 acre-ft of water $1 \mathrm{ft}$ is about $1.8 \mathrm{kWh}$. This value can be used to estimate approximate ground-water pumpage, using powerconsumption data and specific information on total head. 


\section{REFERENCES CITED}

Crowley, J.K., 1986, Visible and near-infrared spectra of carbonate rocks-Reffectance variations related to petrographic textures and impurities: Journal of Geophysical Research, v. 91, no. B5, p. 5001-5012.

Eakin, T.E., 1962, Ground-water appraisal of Diamond Valley, Eureka and Elko Counties, Nevada: Nevada Department of Conservation and Natural Resources, Ground-Water Resources-Reconnaissance Report 6, $60 \mathrm{p}$.

Harrill, J.R., 1968, Hydrologic response to irrigation pumping in Diamond Valley, Eureka and Elko Counties, Nevada, 1950-65: Nevada Department of Conservation and Natural Resources, Water Resources Bulletin 35, $85 \mathrm{p}$.

Heimes, F.J., and Luckey, R.R., 1983, Estimating 1980 ground-water pumpage for irrigation on the High Plains in parts of Colorado, Kansas, Nebraska, New Mexico, Oklahoma, South Dakota, Texas, and Wyoming: U.S. Geological Survey Water-Resources Investigations Report 83-4123, 36 p.

Hoffer, R.M., and Johannsen, C.J., 1969, Ecological potentials in spectral signature and analysis, in Johnson, P.L., ed., Remote sensing in ecology: Athens, University of Georgia Press, p. 1-16.

Lillesand, T.M., and Kiefer, R.W., 1987, Remote sensing and image interpretation: New York, John Wiley, 721 p.
Milton, Nancy, 1978, Spectral reffectance measurements of plants in the East Tintic Mountains, Utah: U.S. Geological Survey Open-File Report 78-448, 123 p.

Moreland, J.A., 1977, Ground water-surface water relations in the Silver Creek area, Blaine County, Idaho: Idaho Department of Water Resources, Water Information Bulletin 44, $42 \mathrm{p}$.

Nevada State Department of Administration, 1990, Nevada statistical abstract-1990 edition: Carson City, Nev., $458 \mathrm{p}$.

Pennington, R.W., 1980, Evaluation of empirical methods for estimating crop water consumptive use for selected sites in Nevada: Nevada Division of Water Planning, Report 3, 206 p.

Podwysocki, M.H., and Segal, D.B., 1983, Use of multispectral scanner images for assessment of hydrothermal alterations in the Marysvale, Utah, mining area: Economic Geology, v. 78, p. 675-687

Rush, F.E., 1968, Index of hydrographic areas in Nevada: Nevada Division of Water Resources, Information Report 6, 38 p.

Tukey, J.W., 1977, Exploratory data analysis: Menlo Park, Calif., Addison-Wesley, 688 p.

U.S. Soil Conservation Service, 1981, Nevada irrigation guide: Reno, Nev., 327 p. 
Appendix 
Appendix. Water-level measurements in selected wells, Diamond Valley, Nevada, 1960-90

Local identification-See section in text, "Numbering System for Wells and Springs," for explanation.

Standard identification--See section in text, "Numbering System for Wells and Springs," for explanation.

Date-Date water level measured; 00 indicates exact day not known.

Water level-Measurements are reported to either nearest hundredth or nearest tenth of a foot, based on accuracy indicated in Ground Water Site Inventory (GWSI) data base.

Status-D, dry or destroyed; P, pumping; S, nearby well is pumping and taps same aquifer ; $Z$, other; --, reported water-level measurement represents static level.

Method-R, data reported but method not known; S, steel-tape measurement by U.S. Geological Survey; Z, measured by Nevada Division of Water Resources; --, method not specified.

\begin{tabular}{|c|c|c|c|c|c|c|c|c|}
\hline \multirow{2}{*}{\multicolumn{3}{|c|}{$\begin{array}{l}\text { U.S. Geological Survey } \\
\text { site designation }\end{array}$}} & \multirow{3}{*}{$\begin{array}{c}\text { Depth } \\
\text { of } \\
\text { well } \\
\text { (feet) }\end{array}$} & \multirow{3}{*}{$\begin{array}{c}\text { Land- } \\
\text { surface } \\
\text { altitude } \\
\text { (feet above } \\
\text { sea level) }\end{array}$} & \multicolumn{4}{|c|}{ Water-level measurement } \\
\hline & & & & & \multirow[b]{2}{*}{ Date } & \multirow{2}{*}{$\begin{array}{c}\text { Feet } \\
\text { below } \\
\text { land } \\
\text { surface }\end{array}$} & \multirow[b]{2}{*}{ Status } & \multirow[b]{2}{*}{ Method } \\
\hline & $\begin{array}{c}\text { Local } \\
\text { identification }\end{array}$ & $\begin{array}{c}\text { Standard } \\
\text { identification }\end{array}$ & & & & & & \\
\hline \multirow[t]{18}{*}{153} & N20 E53 01BDDA 1 & 393731115570301 & 173 & 5,951 & $01-01-60$ & 86.38 & -- & $S$ \\
\hline & & & & & $11-00-61$ & 81.8 & -- & $\mathbf{Z}$ \\
\hline & & & & & $03-00-65$ & 85.7 & -- & Z \\
\hline & & & & & $11-00-65$ & 89.0 & -- & Z \\
\hline & & & & & $03-00-66$ & 86.4 & -- & $\bar{Z}$ \\
\hline & & & & & $03-00-68$ & 92.2 & -- & Z \\
\hline & & & & & $03-00-69$ & 91.8 & -- & $\mathrm{Z}$ \\
\hline & & & & & $11-00-69$ & 101.7 & -- & Z \\
\hline & & & & & $10-01-73$ & 113.5 & -- & Z \\
\hline & & & & & $03-00-74$ & 99.0 & -- & Z \\
\hline & & & & & $11-03-75$ & 107.8 & -- & Z \\
\hline & & & & & $03-01-76$ & 103.0 & -- & Z \\
\hline & & & & & $11-08-76$ & 108.8 & -- & Z \\
\hline & & & & & $03-00-77$ & 102.4 & -- & Z \\
\hline & & & & & $11-14-77$ & 110.6 & -- & $\mathrm{Z}$ \\
\hline & & & & & 03-00-79 & 113.8 & -- & $\mathrm{Z}$ \\
\hline & & & & & $11-08-84$ & 150.0 & -- & $\mathrm{Z}$ \\
\hline & & & & & $10-23-89$ & 132.1 & -- & $\mathrm{Z}$ \\
\hline \multirow[t]{19}{*}{153} & N20 E53 02ACDD 1 & 393724115580201 & 220 & 5,932 & $05-17-66$ & 67.08 & -- & $S$ \\
\hline & & & & & $10.01-73$ & 89.1 & -- & $\mathrm{Z}$ \\
\hline & & & & & $11-03-75$ & 87.6 & -- & $\mathrm{Z}$ \\
\hline & & & & & $03-01-76$ & 82.9 & -- & $\mathrm{Z}$ \\
\hline & & & & & $11-08-76$ & 95.2 & -- & $\mathrm{Z}$ \\
\hline & & & & & $11-14-77$ & 109.8 & -- & $\mathrm{Z}$ \\
\hline & & & & & $11-08-78$ & 112.3 & - & $\mathrm{Z}$ \\
\hline & & & & & $11-01-79$ & 109.8 & -- & $\mathrm{Z}$ \\
\hline & & & & & $11-01-80$ & 112.7 & -- & $\bar{Z}$ \\
\hline & & & & & $11-01-81$ & 125.6 & -- & Z \\
\hline & & & & & $11-09-82$ & 123.6 & -- & Z \\
\hline & & & & & $05-01-83$ & 122.8 & -- & $\mathrm{Z}$ \\
\hline & & & & & $11-09-83$ & 126.8 & -- & $\mathrm{Z}$ \\
\hline & & & & & $11-08-84$ & 118.4 & -- & $\mathbf{Z}$ \\
\hline & & & & & $12-04-85$ & 119.8 &.- & $\mathrm{Z}$ \\
\hline & & & & & $11-05-86$ & 120.0 & - & $\mathrm{Z}$ \\
\hline & & & & & $11-18-87$ & 121.4 & -- & $\mathrm{Z}$ \\
\hline & & & & & $10-20-88$ & 122.1 & -- & Z \\
\hline & & & & & $10-23-89$ & 115.0 & -- & $\mathrm{Z}$ \\
\hline
\end{tabular}


Appendix. Water-level measurements in selected wells, Diamond Valley, Nevada, 1960-90-Continued

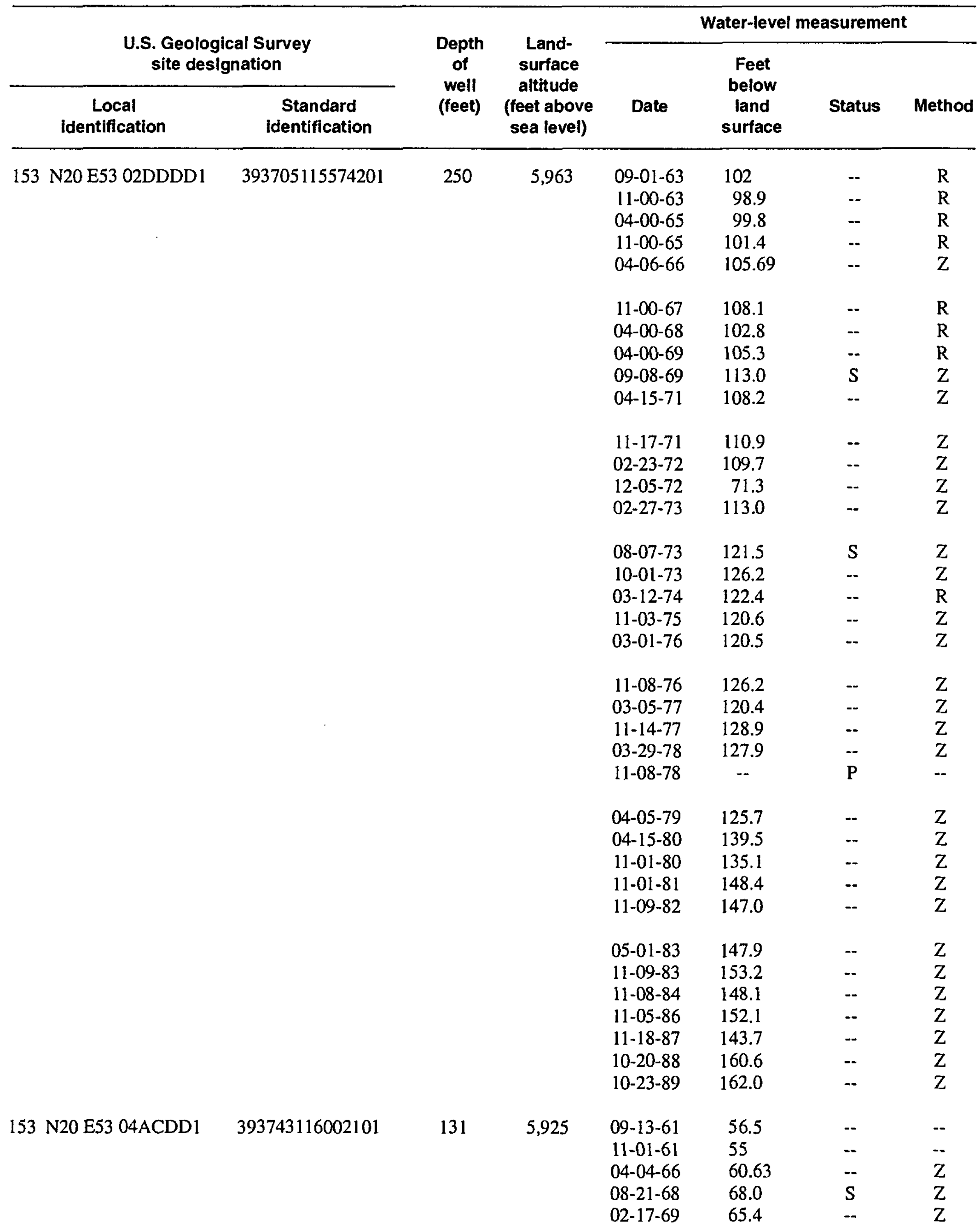


Appendix. Water-level measurements in selected wells, Diamond Valley, Nevada, 1960-90-Continued

\begin{tabular}{|c|c|c|c|c|c|c|c|}
\hline \multirow{2}{*}{\multicolumn{2}{|c|}{$\begin{array}{l}\text { U.S. Geological Survey } \\
\text { site designation }\end{array}$}} & \multirow{3}{*}{$\begin{array}{l}\text { Depth } \\
\text { of } \\
\text { well } \\
\text { (feet) }\end{array}$} & \multirow{3}{*}{$\begin{array}{c}\text { Land- } \\
\text { surface } \\
\text { altitude } \\
\text { (feet above } \\
\text { sea level) }\end{array}$} & \multicolumn{4}{|c|}{ Water-level measurement } \\
\hline & & & & \multirow[b]{2}{*}{ Date } & \multirow{2}{*}{$\begin{array}{c}\text { Feet } \\
\text { below } \\
\text { land } \\
\text { surface }\end{array}$} & \multirow[b]{2}{*}{ Status } & \multirow[b]{2}{*}{ Method } \\
\hline $\begin{array}{c}\text { Local } \\
\text { Identiflcation }\end{array}$ & $\begin{array}{c}\text { Standard } \\
\text { identiflcatlon }\end{array}$ & & & & & & \\
\hline \multirow{21}{*}{\multicolumn{2}{|c|}{153 N20 E53 04ACDD1 (Continued) }} & & & $09-08-69$ & 67.9 & $S$ & $\mathrm{Z}$ \\
\hline & & & & $04-15-71$ & 67.4 & -- & Z \\
\hline & & & & $08-07-73$ & 77.6 & $S$ & $\mathrm{Z}$ \\
\hline & & & & $10-01-73$ & 72.9 & -- & $\mathrm{Z}$ \\
\hline & & & & $11-05-73$ & 73.3 & -- & $\bar{Z}$ \\
\hline & & & & $03-12-74$ & 72.1 & -. & $\mathrm{Z}$ \\
\hline & & & & $03-05-77$ & 78.3 & -- & $\mathrm{Z}$ \\
\hline & & & & $11-14-77$ & 84.4 & -- & $\mathrm{Z}$ \\
\hline & & & & $11-08-78$ & 84.2 & -- & $\mathrm{Z}$ \\
\hline & & & & $04-06-79$ & 83.9 & - & Z \\
\hline & & & & $11-01-79$ & 93.1 & -- & $\mathrm{Z}$ \\
\hline & & & & $11-01-80$ & 95.3 & -- & $\mathrm{Z}$ \\
\hline & & & & $11-01-81$ & 106.1 & -- & $\mathrm{Z}$ \\
\hline & & & & $11-09-82$ & 105.4 & -- & $\mathrm{Z}$ \\
\hline & & & & $05-01-83$ & 98.4 & - & $\mathrm{Z}$ \\
\hline & & & & $11-09-83$ & 99.6 & -- & $\mathrm{Z}$ \\
\hline & & & & $11-08-84$ & 97.9 & - & $\mathrm{Z}$ \\
\hline & & & & $11-05-86$ & 101.0 & -- & $\mathrm{Z}$ \\
\hline & & & & $11-18-87$ & 115.6 & -- & $\mathrm{Z}$ \\
\hline & & & & $10-24-89$ & 107.4 & -- & $\mathrm{Z}$ \\
\hline & & & & $09-13-90$ & -- & $\mathbf{P}$ & -- \\
\hline \multirow[t]{25}{*}{153 N20 E53 04DDBB 1} & 393714116000301 & 177 & 5,928 & $11-00-61$ & 56.5 & -- & $\mathrm{Z}$ \\
\hline & & & & $11-00-63$ & 58.9 & -- & $\mathrm{Z}$ \\
\hline & & & & $11-00-64$ & 66.7 & -- & $\mathrm{Z}$ \\
\hline & & & & $11-00-65$ & 66.2 & -- & $\mathrm{Z}$ \\
\hline & & & & $03-00-66$ & 60.7 & $-\cdot$ & $\mathrm{Z}$ \\
\hline & & & & $04-05-66$ & 60.71 & -- & $\mathrm{S}$ \\
\hline & & & & $11-00-67$ & 65.6 & -- & $\mathrm{Z}$ \\
\hline & & & & $03-00-68$ & 67.9 & -- & $\mathrm{Z}$ \\
\hline & & & & $11-00-68$ & 68.3 & -- & $\mathrm{Z}$ \\
\hline & & & & $03-00-69$ & 65.1 & -- & $\mathrm{Z}$ \\
\hline & & & & $11-00-69$ & 69.3 & -- & $\mathrm{Z}$ \\
\hline & & & & $10-01-73$ & 73.3 & - & $\mathrm{Z}$ \\
\hline & & & & $03-00-74$ & 70.9 & -- & $\mathrm{Z}$ \\
\hline & & & & $11-03-75$ & 63.1 & -- & $\mathrm{Z}$ \\
\hline & & & & $03-01-76$ & 63.4 & - & $\mathrm{Z}$ \\
\hline & & & & $11-08-76$ & 64.0 & -- & $\mathrm{Z}$ \\
\hline & & & & $11-14-77$ & 67.8 & -- & $\mathrm{Z}$ \\
\hline & & & & $11-08-78$ & 70.1 & -- & $\mathrm{Z}$ \\
\hline & & & & $03-00-79$ & 82.8 & -- & $\mathrm{Z}$ \\
\hline & & & & $11-01-79$ & 82.8 & -- & $\mathrm{Z}$ \\
\hline & & & & $11-01-80$ & 88.5 & -- & $\mathrm{Z}$ \\
\hline & & & & $11-01-81$ & 93.2 & -- & $\mathrm{Z}$ \\
\hline & & & & $11-09-82$ & 91.6 & -- & $\mathrm{Z}$ \\
\hline & & & & $05-01-83$ & 93.8 & - & $\mathrm{Z}$ \\
\hline & & & & $09-13-90$ & -- & $\mathbf{P}$ & -- \\
\hline
\end{tabular}


Appendix. Water-level measurements in selected wells, Diamond Valley, Nevada, 1960-90-Continued

\begin{tabular}{|c|c|c|c|c|c|c|c|c|}
\hline \multirow{2}{*}{\multicolumn{3}{|c|}{$\begin{array}{l}\text { U.S. Geological Survey } \\
\text { site designation }\end{array}$}} & \multirow{3}{*}{$\begin{array}{c}\text { Depth } \\
\text { of } \\
\text { well } \\
\text { (feet) }\end{array}$} & \multirow{3}{*}{$\begin{array}{l}\text { Land- } \\
\text { surface } \\
\text { altitude } \\
\text { (feet above } \\
\text { sea level) }\end{array}$} & \multicolumn{4}{|c|}{ Water-level measurement } \\
\hline & & & & & \multirow[b]{2}{*}{ Date } & \multirow{2}{*}{$\begin{array}{l}\text { Feet } \\
\text { below } \\
\text { land } \\
\text { surface }\end{array}$} & \multirow[b]{2}{*}{ Status } & \multirow[b]{2}{*}{ Method } \\
\hline & $\begin{array}{l}\text { Local } \\
\text { Identification }\end{array}$ & $\begin{array}{l}\text { Standard } \\
\text { identification }\end{array}$ & & & & & & \\
\hline \multirow{29}{*}{\multicolumn{2}{|c|}{153 N20 E53 10ADDD1 }} & 393645115585801 & 180 & 5,947 & $11-00-61$ & 72.5 & - & $\mathrm{Z}$ \\
\hline & & & & & $11-00-63$ & 76.8 & -- & $\mathrm{Z}$ \\
\hline & & & & & $03-00-64$ & 76.5 & -- & $\mathrm{Z}$ \\
\hline & & & & & $11-00-64$ & 79.4 & -- & $\mathrm{Z}$ \\
\hline & & & & & $11-00-65$ & 80.0 & -- & Z \\
\hline & & & & & $03-00-66$ & 83.1 & -- & $\mathrm{Z}$ \\
\hline & & & & & $04-05-66$ & 83.05 & -- & $\mathrm{s}$ \\
\hline & & & & & $11-00-67$ & 84.8 & -- & $\mathrm{Z}$ \\
\hline & & & & & $03-00-68$ & 87.8 & -- & $\mathrm{Z}$ \\
\hline & & & & & 03-00-69 & 83.6 & -- & $\mathrm{z}$ \\
\hline & & & & & $11-00-69$ & 89.8 & -. & $\mathrm{z}$ \\
\hline & & & & & $11-00-71$ & 83.3 & - & $\mathrm{Z}$ \\
\hline & & & & & $03-00-72$ & 88.3 & -- & $\mathrm{Z}$ \\
\hline & & & & & $11-00-72$ & 90.6 & .- & $\mathrm{z}$ \\
\hline & & & & & $03-00-73$ & 95.3 & -- & Z \\
\hline & & & & & $10-01-73$ & 106.1 & -. & $\mathrm{Z}$ \\
\hline & & & & & $03-00-74$ & 98.2 & -- & $\mathrm{Z}$ \\
\hline & & & & & $11-03-75$ & 102.5 & -- & $\mathrm{Z}$ \\
\hline & & & & & $03-0 i-76$ & 97.8 & -- & $\mathrm{Z}$ \\
\hline & & & & & $11-08-76$ & 101.4 & -- & $\mathrm{Z}$ \\
\hline & & & & & $03-00-77$ & 99.5 & -- & $\mathrm{z}$ \\
\hline & & & & & $11-14-77$ & 105.3 & -. & $\mathrm{Z}$ \\
\hline & & & & & $03-00-78$ & 101.1 & -- & $\mathrm{Z}$ \\
\hline & & & & & $03-00-79$ & 101.8 & -- & $\mathrm{Z}$ \\
\hline & & & & & $11-01-79$ & 111.4 & -. & $\mathrm{Z}$ \\
\hline & & & & & $11-01-80$ & 113.3 & _- & $\mathrm{Z}$ \\
\hline & & & & & $11-01-81$ & 127.9 & -- & $\mathrm{Z}$ \\
\hline & & & - & & $05-01-83$ & 133.4 & -. & $\mathrm{Z}$ \\
\hline & & & & & $11-09-83$ & 135.6 & -- & $\mathrm{Z}$ \\
\hline \multirow{16}{*}{\multicolumn{2}{|c|}{153 N20 E53 10BADD1 }} & 393645115592801 & 220 & 5,936 & $04-05-66$ & 67.44 & -- & 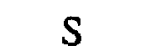 \\
\hline & & & & & $10-01-73$ & 98.3 & -- & $\mathrm{Z}$ \\
\hline & & & & & $11-03-75$ & 86.5 & -- & $\mathrm{Z}$ \\
\hline & & & & & $03-01-76$ & 84.4 & -- & Z \\
\hline & & & & & $11-08-76$ & 91.0 & -- & Z \\
\hline & & & & & $11-14-77$ & -- & $\mathrm{P}$ & -- \\
\hline & & & & & $11-08-78$ & -. & $\mathbf{P}$ & -- \\
\hline & & & & & $11-01.79$ & 100.8 & -- & $\mathrm{Z}$ \\
\hline & & & & & $11-01-80$ & 108.7 & -- & $\mathrm{Z}$ \\
\hline & & & & & $11-01-81$ & 115.1 & - & $\mathrm{Z}$ \\
\hline & & & & & $11-09-82$ & 106.7 & -- & $\mathrm{Z}$ \\
\hline & & & & & $05-01-83$ & 114.0 & -- & $\mathrm{Z}$ \\
\hline & & & & & $11-09-83$ & 107.3 & -- & $\mathrm{z}$ \\
\hline & & & & & $11-07-84$ & 112.6 & -- & $\mathrm{Z}$ \\
\hline & & & & & $11-05-86$ & 113.0 & -- & $\mathrm{Z}$ \\
\hline & & & & & $10-24-89$ & 116.9 & -- & $\mathrm{Z}$ \\
\hline
\end{tabular}


Appendix. Water-level measurements in selected wells, Diamond Valley, Nevada, 1960-90—Continued

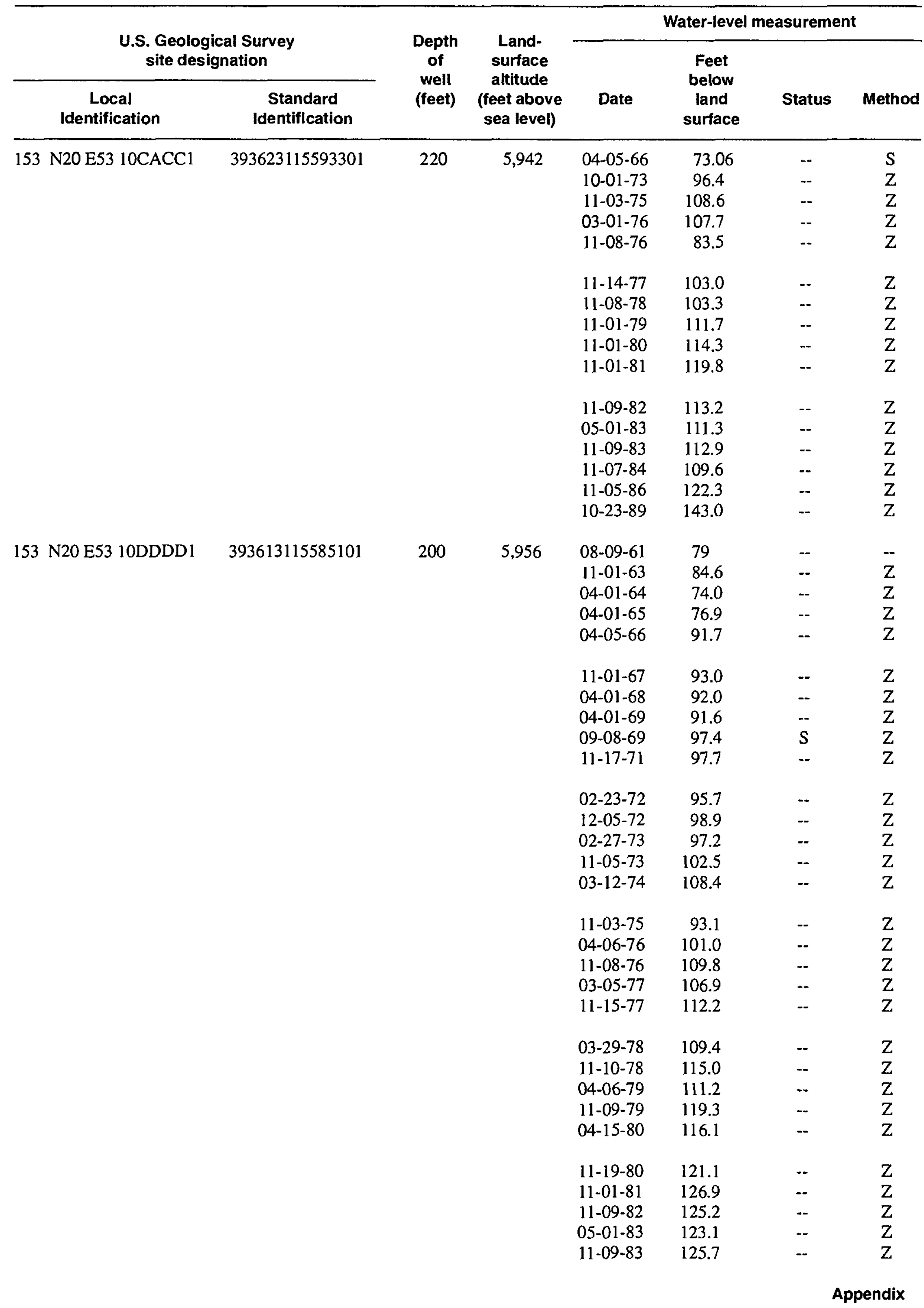


Appendix. Water-level measurements in selected wells, Diamond Valley, Nevada, 1960-90-Continued

\begin{tabular}{|c|c|c|c|c|c|c|c|c|}
\hline \multirow{2}{*}{\multicolumn{3}{|c|}{$\begin{array}{c}\text { U.S. Geological Survey } \\
\text { aite designation }\end{array}$}} & \multirow{3}{*}{$\begin{array}{c}\text { Depth } \\
\text { of } \\
\text { well } \\
\text { (feet) }\end{array}$} & \multirow{3}{*}{$\begin{array}{l}\text { Land- } \\
\text { surface } \\
\text { altitude } \\
\text { (feet above } \\
\text { sea level) }\end{array}$} & \multicolumn{4}{|c|}{ Water-level measurement } \\
\hline & & & & & \multirow[b]{2}{*}{ Date } & \multirow{2}{*}{$\begin{array}{c}\text { Feet } \\
\text { below } \\
\text { land } \\
\text { surface }\end{array}$} & \multirow[b]{2}{*}{ Status } & \multirow[b]{2}{*}{ Method } \\
\hline & $\begin{array}{c}\text { Local } \\
\text { Identification }\end{array}$ & $\begin{array}{l}\text { Standard } \\
\text { identification }\end{array}$ & & & & & & \\
\hline \multirow[t]{8}{*}{153} & N20 E53 10DDDD1 & (Continued) & & & $12-04-85$ & 126.3 & -- & $Z$ \\
\hline & & & & & $11-05-86$ & 129.8 & -- & $\mathrm{Z}$ \\
\hline & & & & & $11-18-87$ & 132.7 & -- & $\mathrm{Z}$ \\
\hline & & & & & $03-30-88$ & 130.8 & -- & $\mathrm{Z}$ \\
\hline & & & & & $10-20-88$ & 141.3 & -- & Z \\
\hline & & & & & $04-05-89$ & 138.7 & -- & $\mathrm{Z}$ \\
\hline & & & & & $10-23-89$ & 131.3 & -- & $\mathrm{Z}$ \\
\hline & & & & & $11-28-90$ & 142.0 & -- & $\mathrm{Z}$ \\
\hline \multirow[t]{5}{*}{153} & N20 E53 11ACCD1 & 393647115582601 & 162 & 5,970 & $04-05-66$ & 103.01 & -- & $\mathrm{s}$ \\
\hline & & & & & $11-03-75$ & 117.5 & -- & $\mathrm{Z}$ \\
\hline & & & & & $11-08-76$ & 125.8 & -- & $\mathrm{Z}$ \\
\hline & & & & & $11-14-77$ & 125.5 & -- & Z \\
\hline & & & & & $11-01-80$ & 138.6 & -- & $\mathrm{Z}$ \\
\hline \multirow[t]{12}{*}{153} & N20 E53 11 ADCCl & 393647115574901 & -- & 5,965 & $04-05-66$ & 100.04 & -- & $S$ \\
\hline & & & & & $11-01-80$ & 139.8 & -- & $\mathrm{Z}$ \\
\hline & & & & & $11-01-81$ & 134.3 & -- & $Z$ \\
\hline & & & & & $11-09-82$ & 131.1 & -- & $Z$ \\
\hline & & & & & $05-01-83$ & 129.3 & -. & $\mathrm{Z}$ \\
\hline & & & & & $11-09-83$ & 131.0 & .- & $\mathrm{Z}$ \\
\hline & & & & & $11-08-84$ & 129.5 & -- & $\mathrm{Z}$ \\
\hline & & & & & $12-04-85$ & 135.4 & -- & Z \\
\hline & & & & & $11-05-86$ & 139.5 & -- & $\mathrm{Z}$ \\
\hline & & & & & $11-18-87$ & 145.3 & -- & $Z$ \\
\hline & & & & & $10-20-88$ & -- & $\mathbf{P}$ & -- \\
\hline & & & & & $10-23-89$ & 147.1 & -- & $z$ \\
\hline \multirow[t]{17}{*}{$153 N$} & N20 E53 11CDBB 1 & 393623115582401 & 300 & 5,957 & $04-05-66$ & 91.36 & -. & $S$ \\
\hline & & & & & $10-01-73$ & 102.3 & -- & $\mathrm{Z}$ \\
\hline & & & & & $11-03-75$ & 107.8 & -- & $z$ \\
\hline & & & & & $03-01-76$ & 112.1 & -- & Z \\
\hline & & & & & $11-08-76$ & 110.7 & -- & $\mathrm{Z}$ \\
\hline & & & & & $11-14-77$ & 112.6 & -- & Z \\
\hline & & & & & $11-08-78$ & 115.7 & -- & Z \\
\hline & & & & & $11-01-79$ & 120.1 & -- & Z \\
\hline & & & & & $11-01-80$ & 123.0 & -- & Z \\
\hline & & & & & $11-01-81$ & 131.6 & -- & Z \\
\hline & & & & & $11-09-82$ & 124.3 & -- & Z \\
\hline & & & & & $05-01-83$ & 133.3 & -- & Z \\
\hline & & & & & $11-09-83$ & 137.1 & -- & Z \\
\hline & & & & & $11-07-84$ & 127.1 & -- & Z \\
\hline & & & & & $11-18-87$ & 136.1 & -- & Z \\
\hline & & & & & $10-20-88$ & 142.9 & -- & Z \\
\hline & & & & & $10-23-89$ & 133.7 & -. & Z \\
\hline
\end{tabular}


Appendix. Water-level measurements in selected wells, Diamond Valley, Nevada, 1960-90-Continued

\begin{tabular}{|c|c|c|c|c|c|c|c|}
\hline \multirow{2}{*}{\multicolumn{2}{|c|}{$\begin{array}{l}\text { U.S. Geological Survey } \\
\text { site designation }\end{array}$}} & \multirow{3}{*}{$\begin{array}{c}\text { Depth } \\
\text { of } \\
\text { well } \\
\text { (feet) }\end{array}$} & \multirow{3}{*}{$\begin{array}{c}\text { Land- } \\
\text { surface } \\
\text { altitude } \\
\text { (feet above } \\
\text { sea level) }\end{array}$} & \multicolumn{4}{|c|}{ Water-level measurement } \\
\hline & & & & \multirow[b]{2}{*}{ Date } & \multirow{2}{*}{$\begin{array}{c}\text { Feet } \\
\text { below } \\
\text { land } \\
\text { surface }\end{array}$} & \multirow[b]{2}{*}{ Status } & \multirow[b]{2}{*}{ Method } \\
\hline $\begin{array}{l}\text { Local } \\
\text { Identlflcation }\end{array}$ & $\begin{array}{c}\text { Standard } \\
\text { Identification }\end{array}$ & & & & & & \\
\hline \multirow[t]{8}{*}{153 N20 E53 11DDBB1 } & 393623115575801 & -- & 5,967 & $10-01-73$ & 115.1 & -- & $\mathbf{Z}$ \\
\hline & & & & $11-03-75$ & 117.8 & $\cdots$ & $\mathrm{Z}$ \\
\hline & & & & $03-01-76$ & 115.3 & -- & $\mathbf{Z}$ \\
\hline & & & & $11-08-76$ & 120.0 & -- & $\mathrm{Z}$ \\
\hline & & & & $11-14-77$ & 122.9 & -- & $\mathrm{Z}$ \\
\hline & & & & $11-08-78$ & 125.1 & -- & $\mathrm{Z}$ \\
\hline & & & & $11-01-79$ & 129.2 & -- & $\mathbf{Z}$ \\
\hline & & & & $11-01-80$ & 131.5 & -- & Z \\
\hline \multirow[t]{36}{*}{153 N20 E53 15CDDD1 } & 393519115592401 & 398 & 5,996 & $11-00-65$ & 122.2 & -- & $\mathrm{R}$ \\
\hline & & & & $04-05-66$ & 121.54 & -- & $\mathbf{Z}$ \\
\hline & & & & $11-00-66$ & 137.8 & -- & $\mathbf{R}$ \\
\hline & & & & $04-00-67$ & 126.3 & -- & $\mathrm{R}$ \\
\hline & & & & $11-00-67$ & 135.4 & -- & $\mathbf{R}$ \\
\hline & & & & $04-00-68$ & 131.0 & -- & $\mathbf{R}$ \\
\hline & & & & $04-00-69$ & 125.8 & -- & $\mathrm{R}$ \\
\hline & & & & $09-08-69$ & 129.5 & $S$ & Z \\
\hline & & & & $04-15-71$ & 128.8 & -- & $\mathbf{Z}$ \\
\hline & & & & $11-17-71$ & 132.2 & -- & $\mathrm{Z}$ \\
\hline & & & & $02-23-72$ & 129.0 & -- & $\mathbf{Z}$ \\
\hline & & & & $12-05-72$ & 132.8 & -- & $\mathrm{Z}$ \\
\hline & & & & $02-27-73$ & 132.2 & -- & $\mathbf{Z}$ \\
\hline & & & & $10-01-73$ & 141.1 & -- & $\mathbf{Z}$ \\
\hline & & & & $03-12-74$ & 139.0 & -- & $\mathrm{Z}$ \\
\hline & & & & $11-03-75$ & 147.4 & -. & $\mathbf{Z}$ \\
\hline & & & & $03-01-76$ & 143.0 & -- & Z \\
\hline & & & & $11-08-76$ & 155.9 & -- & $\mathrm{Z}$ \\
\hline & & & & $03-05-77$ & 142.4 & $\cdots$ & $\mathrm{Z}$ \\
\hline & & & & $11-14-77$ & 161.3 & -- & $\mathrm{Z}$ \\
\hline & & & & $03-29-78$ & 157.1 & -- & $\mathbf{Z}$ \\
\hline & & & & $11-08-78$ & 159.7 & -- & $\mathrm{Z}$ \\
\hline & & & & $04-06-79$ & 156.9 & -- & $\mathrm{Z}$ \\
\hline & & & & $11-01-79$ & 162.8 & -- & $\mathrm{Z}$ \\
\hline & & & & $04-15-80$ & 159.2 & -- & $\mathbf{Z}$ \\
\hline & & & & $11-01-80$ & 161.9 & -- & $\mathbf{Z}$ \\
\hline & & & & $11-01-81$ & 169.2 & -- & $\mathrm{Z}$ \\
\hline & & & & $11-09-82$ & 168.5 & -- & $\mathbf{Z}$ \\
\hline & & & & $05-01-83$ & 167.1 & -- & $\mathbf{Z}$ \\
\hline & & & & $11-09-83$ & 169.9 & -- & $\mathrm{Z}$ \\
\hline & & & & $11-07-84$ & 170.0 & -- & $\mathbf{Z}$ \\
\hline & & & & $12-04-85$ & 170.4 & -- & $\mathrm{Z}$ \\
\hline & & & & $11-05-86$ & 171.0 & -- & $\mathbf{Z}$ \\
\hline & & & & $11-18-87$ & 173.8 & $\cdots$ & $\mathrm{Z}$ \\
\hline & & & & $10-20-88$ & 179.5 & $\cdots$ & Z \\
\hline & & & & $10-23-89$ & 180.4 & -- & $\mathbf{Z}$ \\
\hline
\end{tabular}


Appendix. Water-level measurements in selected wells, Diamond Valley, Nevada, 1960-90-Continued

\begin{tabular}{|c|c|c|c|c|c|c|c|}
\hline \multirow{2}{*}{\multicolumn{2}{|c|}{$\begin{array}{l}\text { U.S. Geological Survey } \\
\text { site designation }\end{array}$}} & \multirow{3}{*}{$\begin{array}{l}\text { Depth } \\
\text { of } \\
\text { well } \\
\text { (feet) }\end{array}$} & \multirow{3}{*}{$\begin{array}{c}\text { Land- } \\
\text { surface } \\
\text { altitude } \\
\text { (feet above } \\
\text { sea level) }\end{array}$} & \multicolumn{4}{|c|}{ Water-level measurement } \\
\hline & & & & \multirow[b]{2}{*}{ Date } & \multirow{2}{*}{$\begin{array}{l}\text { Feet } \\
\text { below } \\
\text { land } \\
\text { surface }\end{array}$} & \multirow[b]{2}{*}{ Status } & \multirow[b]{2}{*}{ Method } \\
\hline $\begin{array}{l}\text { Local } \\
\text { identification }\end{array}$ & $\begin{array}{l}\text { Standard } \\
\text { Identification }\end{array}$ & & & & & & \\
\hline \multirow[t]{3}{*}{153 N20 E53 17BCCC1 } & 393545116021101 & -- & 5,946 & $11-08-76$ & 59.7 & -- & $\mathrm{Z}$ \\
\hline & & & & $11-14-77$ & 64.1 & -- & $\mathrm{Z}$ \\
\hline & & & & $10-25-90$ & 79.0 & -- & $\mathrm{Z}$ \\
\hline \multirow[t]{39}{*}{153 N20 E53 18DCCC1 } & 393522116024501 & 165 & 5,954 & $04-03-62$ & 44.0 & -- & $\mathbf{R}$ \\
\hline & & & & $11-00-63$ & 46.5 & -- & $\mathrm{R}$ \\
\hline & & & & $04-00-64$ & 59.5 & -- & $\mathbf{R}$ \\
\hline & & & & $04-00-65$ & 42.6 & - & $\mathrm{R}$ \\
\hline & & & & $11-00-65$ & 47.5 & -- & $\mathrm{R}$ \\
\hline & & & & $04-04-66$ & 48.19 & -- & $\mathrm{Z}$ \\
\hline & & & & $11-00-66$ & 55.9 & -- & $\mathbf{R}$ \\
\hline & & & & $04-00-67$ & 56.9 & -- & $\mathrm{R}$ \\
\hline & & & & $11-00-67$ & 52.5 & .. & $\mathbf{R}$ \\
\hline & & & & $04-00-68$ & 50.8 & -- & $\mathbf{R}$ \\
\hline & & & & $04-00-69$ & 52.6 & -- & $\mathbf{R}$ \\
\hline & & & & $09-08-69$ & 53.7 & $\mathrm{~S}$ & $\mathrm{Z}$ \\
\hline & & & & $04-14-7 \mid$ & -- & $\mathbf{P}$ & -- \\
\hline & & & & $11-17-71$ & 55.0 & -- & $\mathrm{z}$ \\
\hline & & & & $02-23-72$ & 65.8 & -- & $\mathrm{Z}$ \\
\hline & & & & $12-05-72$ & 66.1 & -- & $\mathrm{Z}$ \\
\hline & & & & $02-27-73$ & 64.1 & -- & $\mathrm{z}$ \\
\hline & & & & $08-07-73$ & -- & $P$ & -- \\
\hline & & & & $11-06-73$ & 64.2 & -- & $\mathrm{Z}$ \\
\hline & & & & $03-12-74$ & 63.6 & -- & $\mathrm{Z}$ \\
\hline & & & & $11-04-75$ & 59.0 & -- & $\mathrm{Z}$ \\
\hline & & & & $03-05-77$ & 60.6 & -- & $\mathrm{Z}$ \\
\hline & & & & $11-15-77$ & 57.4 & -- & $\mathrm{Z}$ \\
\hline & & & & $03-30-78$ & 70.2 & -- & Z \\
\hline & & & & $11-10-78$ & 65.7 & -- & $\mathrm{Z}$ \\
\hline & & & & $04-06-79$ & 63.6 & -- & $\mathrm{Z}$ \\
\hline & & & & $11-09-79$ & 69.0 & -. & Z \\
\hline & & & & $04-15-80$ & 63.5 & -- & Z \\
\hline & & & & $11-19-80$ & 70.0 & -- & Z \\
\hline & & & & $11-01-81$ & 83.9 & -- & $\mathrm{z}$ \\
\hline & & & & $11-09-82$ & 79.3 & -- & Z \\
\hline & & & & $05-01-83$ & 82.7 & -- & $\mathrm{Z}$ \\
\hline & & & & $11-09-83$ & 81.1 & -- & $\mathrm{Z}$ \\
\hline & & & & $11-07-84$ & 80.2 & -- & Z \\
\hline & & & & $12-03-85$ & 79.9 & -- & $\mathrm{Z}$ \\
\hline & & & & $11-05-86$ & 79.8 & -- & $z$ \\
\hline & & & & $11-18-87$ & 81.4 & -- & Z \\
\hline & & & & $10-20-88$ & 81.0 & -. & $\mathrm{Z}$ \\
\hline & & & & $10-23-89$ & 75.7 & -- & $\mathrm{Z}$ \\
\hline
\end{tabular}


Appendix. Water-level measurements in selected wells, Diamond Valley, Nevada, 1960-90-Continued

\begin{tabular}{|c|c|c|c|c|c|c|c|c|}
\hline \multirow{2}{*}{\multicolumn{3}{|c|}{$\begin{array}{l}\text { U.S. Geological Survey } \\
\text { site designatlon }\end{array}$}} & \multirow{3}{*}{$\begin{array}{c}\text { Depth } \\
\text { of } \\
\text { well } \\
\text { (feet) }\end{array}$} & \multirow{3}{*}{$\begin{array}{l}\text { Land- } \\
\text { surface } \\
\text { altitude } \\
\text { (feet above } \\
\text { sea levei) }\end{array}$} & \multicolumn{4}{|c|}{ Water-level measurement } \\
\hline & & & & & \multirow[b]{2}{*}{ Date } & \multirow{2}{*}{$\begin{array}{c}\text { Feet } \\
\text { below } \\
\text { land } \\
\text { surface }\end{array}$} & \multirow[b]{2}{*}{ Status } & \multirow[b]{2}{*}{ Method } \\
\hline & $\begin{array}{l}\text { Local } \\
\text { identification }\end{array}$ & $\begin{array}{l}\text { Standard } \\
\text { identification }\end{array}$ & & & & & & \\
\hline \multirow[t]{9}{*}{153} & N20 E53 20ADCB1 & 393509116011801 & 275 & 5,966 & $04-04-66$ & 97.06 & -- & $\mathrm{S}$ \\
\hline & & & & & $10-01-73$ & 113.9 & -- & $\mathrm{Z}$ \\
\hline & & & & & $11-03-75$ & 119.8 & -- & $\mathrm{Z}$ \\
\hline & & & & & $11-08-76$ & 129.9 & -- & Z \\
\hline & & & & & $11-14-77$ & 133.7 & -- & $\mathrm{z}$ \\
\hline & & & & & $11-08-78$ & 136.5 & -- & $z$ \\
\hline & & & & & $11-01-79$ & 143.5 & -- & $\mathrm{Z}$ \\
\hline & & & & & $11-01-80$ & 152.1 & -- & $\mathrm{Z}$ \\
\hline & & & & & $11-01-81$ & 138.5 & -. & $\mathrm{Z}$ \\
\hline \multirow[t]{7}{*}{153} & $\mathrm{~N} 20$ E53 20CCAB1 & 393453116014201 & 200 & 5,961 & $04-04-66$ & 91.83 & -. & $\mathrm{S}$ \\
\hline & & & & & $10-01-73$ & 104.9 & -. & $\mathrm{Z}$ \\
\hline & & & & & $11-03-75$ & 109.3 & -- & $\mathrm{Z}$ \\
\hline & & & & & $03-01-76$ & 102.7 & -- & $\mathrm{Z}$ \\
\hline & & & & & $11-08-76$ & 117.3 & -- & $\mathrm{Z}$ \\
\hline & & & & & $11-14-77$ & 120.4 & -- & $\mathrm{Z}$ \\
\hline & & & & & $11-08-78$ & 123.1 & -- & $\mathrm{Z}$ \\
\hline \multirow[t]{9}{*}{153} & N20 E53 20DCAA1 & 393442116010701 & 200 & 5,960 & 04-04-66 & 91.64 & -- & 5 \\
\hline & & & & & $\begin{array}{l}10-01-73 \\
11-03-75\end{array}$ & $\begin{array}{l}106.5 \\
112.6\end{array}$ & -- & $\begin{array}{l}Z \\
Z\end{array}$ \\
\hline & & & & & $03-01-76$ & 107.0 & - & $\mathrm{Z}$ \\
\hline & & & & & $11-08-76$ & 116.4 & -- & $\mathrm{Z}$ \\
\hline & & & & & $11-14-77$ & 118.3 & -- & $\mathrm{z}$ \\
\hline & & & & & $11-08-78$ & 120.2 & -- & $\mathrm{Z}$ \\
\hline & & & & & $11-01-79$ & 128.2 & -- & $\bar{z}$ \\
\hline & & & & & $11-01-80$ & 129.5 & -- & $\mathrm{Z}$ \\
\hline & & & & & $11-01-81$ & 135.2 & -- & $\mathrm{Z}$ \\
\hline \multirow[t]{21}{*}{153} & N20 E53 21AACC1 & 393455116001301 & 200 & 5,974 & $03-05-62$ & 99 & -- & $\mathrm{R}$ \\
\hline & & & & & $04-04-66$ & 98.29 & .- & $\mathrm{Z}$ \\
\hline & & & & & $11-00-66$ & 101.3 & -- & $\mathbf{R}$ \\
\hline & & & & & $04-00-67$ & 104.7 & -- & $\mathbf{R}$ \\
\hline & & & & & $11-00-67$ & 124.8 & - & $\mathbf{R}$ \\
\hline & & & & & $04-00-68$ & 120.8 & -. & $\mathbf{R}$ \\
\hline & & & & & $08-21-68$ & 105.6 & $S$ & Z \\
\hline & & & & & $04-00-69$ & 102.7 & -. & $\mathrm{R}$ \\
\hline & & & & & $09-08-69$ & 105.1 & $\mathrm{~S}$ & Z \\
\hline & & & & & $04-15-71$ & 105.8 & - & $\bar{z}$ \\
\hline & & & & & $11-17-71$ & 119.5 & - & $\mathrm{Z}$ \\
\hline & & & & & $02-23-72$ & 107.6 & -- & $\mathrm{z}$ \\
\hline & & & & & $12-05-72$ & 111.1 & - & Z \\
\hline & & & & & $02-27-73$ & 111.1 & -- & Z \\
\hline & & & & & $08-08-73$ & 117.5 & $\mathrm{~S}$ & Z \\
\hline & & & & & $10-01-73$ & 123.3 & -- & $\mathrm{Z}$ \\
\hline & & & & & $03-12-74$ & 110.9 & -- & Z \\
\hline & & & & & $11-03-75$ & 123.1 & -. & Z \\
\hline & & & & & $03-01-76$ & 124.8 & -- & Z \\
\hline & & & & & $11-08-76$ & 129.6 & -- & z \\
\hline & & & & & & & & ndix \\
\hline
\end{tabular}


Appendix. Water-level measurements in selected wells, Diamond Valley, Nevada, 1960-90—Continued

\begin{tabular}{|c|c|c|c|c|c|c|c|c|}
\hline \multirow{2}{*}{\multicolumn{3}{|c|}{$\begin{array}{l}\text { U.S. Geological Survey } \\
\text { site designation }\end{array}$}} & \multirow{3}{*}{$\begin{array}{l}\text { Depth } \\
\text { of } \\
\text { well } \\
\text { (feet) }\end{array}$} & \multirow{3}{*}{$\begin{array}{l}\text { Land- } \\
\text { surface } \\
\text { altitude } \\
\text { (feet above } \\
\text { sea level) }\end{array}$} & \multicolumn{4}{|c|}{ Water-level measurement } \\
\hline & & & & & \multirow[b]{2}{*}{ Date } & \multirow{2}{*}{$\begin{array}{c}\text { Feet } \\
\text { below } \\
\text { land } \\
\text { surface }\end{array}$} & \multirow[b]{2}{*}{ Status } & \multirow[b]{2}{*}{ Method } \\
\hline & $\begin{array}{l}\text { Local } \\
\text { identification }\end{array}$ & $\begin{array}{l}\text { Standard } \\
\text { identification }\end{array}$ & & & & & & \\
\hline \multirow{10}{*}{\multicolumn{2}{|c|}{$153 \mathrm{~N} 20 \mathrm{E} 5321 \mathrm{AACCl}$}} & Continued) & & & 03-05-77 & 119.70 & -- & $\mathrm{z}$ \\
\hline & & & & & $11-14-77$ & 132.6 & -- & $\mathrm{Z}$ \\
\hline & & & & & $03-30-78$ & 126.8 & -- & $z$ \\
\hline & & & & & $11-08-78$ & 130.3 & -- & $\mathrm{Z}$ \\
\hline & & & & & $04-06-79$ & 123.3 & -. & $\mathrm{Z}$ \\
\hline & & & & & $11-09-79$ & 136.3 & -- & $\mathrm{Z}$ \\
\hline & & & & & $04-15-80$ & 130.1 & -- & $\mathrm{z}$ \\
\hline & & & & & $11-01-80$ & 135.5 & -- & $\mathrm{Z}$ \\
\hline & & & & & $11-00-81$ & 142.8 & -- & $\mathbf{R}$ \\
\hline & & & & & $03-31-83$ & 150.6 & -- & $\mathrm{Z}$ \\
\hline \multirow{16}{*}{\multicolumn{2}{|c|}{153 N20 E53 21BDDD1 }} & 393505116003501 & 200 & 5,966 & $04-04-66$ & 98.29 & -. & $\mathrm{S}$ \\
\hline & & & & & $11-03-75$ & 119.9 & -- & $z$ \\
\hline & & & & & $03-01-76$ & 116.4 & -. & $\mathrm{Z}$ \\
\hline & & & & & $11-08-76$ & 122.4 & -- & $\mathrm{Z}$ \\
\hline & & & & & $11-14-77$ & 128.7 & -- & $z$ \\
\hline & & & & & $11-08-78$ & 124.0 & -. & $z$ \\
\hline & & & & & $11-01-79$ & 136.3 & -- & $\mathrm{Z}$ \\
\hline & & & & & $11-01-80$ & 135.4 & -- & $\mathrm{Z}$ \\
\hline & & & & & $11-01-81$ & 148.2 & -- & $\mathrm{Z}$ \\
\hline & & & & & $11-09-82$ & 151.4 & -- & $\mathrm{z}$ \\
\hline & & & & & $05-01-83$ & 150.6 & -- & $\mathrm{Z}$ \\
\hline & & & & & $11-09-83$ & 152.6 & -- & $Z$ \\
\hline & & & & & $11-05-86$ & 153.1 & -. & $\mathrm{Z}$ \\
\hline & & & & & $11-18-87$ & 154.0 & -- & $z$ \\
\hline & & & & & $10-20-88$ & 157.9 & -- & $\mathrm{Z}$ \\
\hline & & & & & $10-23-89$ & 151.5 & -- & $z$ \\
\hline \multirow[t]{17}{*}{1531} & N20 E53 21CDDC1 & 393440116001901 & 248 & 5,974 & $04-04-66$ & 124.29 & $\ldots$ & S \\
\hline & & & & & $10-01-73$ & 120.8 & -- & $\mathrm{Z}$ \\
\hline & & & & & $11-03-75$ & 128.0 & -- & $Z$ \\
\hline & & & & & $11-08-76$ & 132.8 & -- & $\mathrm{Z}$ \\
\hline & & & & & $11-14-77$ & 134.2 & -- & $\mathrm{Z}$ \\
\hline & & & & & $11-08-78$ & 136.8 & -- & $\mathrm{Z}$ \\
\hline & & & & & $11-01-79$ & 141.1 & -- & $\mathrm{Z}$ \\
\hline & & & & & $11-01-80$ & 142.8 & -- & $\mathrm{Z}$ \\
\hline & & & & & $11-01-81$ & 146.3 & -- & Z \\
\hline & & & & & $11-09-82$ & 147.0 & -- & $\mathrm{Z}$ \\
\hline & & & & & $05-01-83$ & 145.3 & -- & $\mathrm{z}$ \\
\hline & & & & & $11-09-83$ & 150.8 & -- & Z \\
\hline & & & & & $11-07-84$ & -- & $P$ & -- \\
\hline & & & & & $11-05-86$ & 151.0 & -- & $\mathrm{Z}$ \\
\hline & & & & & $11-18-87$ & 152.8 & -- & Z \\
\hline & & & & & $10-20-88$ & 156.5 & -- & Z \\
\hline & & & & & $10-23-89$ & 154.8 & -- & $\mathrm{Z}$ \\
\hline
\end{tabular}


Appendix. Water-level measurements in selected wells, Diamond Valley, Nevada, 1960-90-Continued

\begin{tabular}{|c|c|c|c|c|c|c|c|c|}
\hline \multirow{2}{*}{\multicolumn{3}{|c|}{$\begin{array}{c}\text { U.S. Geological Survey } \\
\text { site designation }\end{array}$}} & \multirow{3}{*}{$\begin{array}{c}\text { Depth } \\
\text { of } \\
\text { well } \\
\text { (feet) }\end{array}$} & \multirow{3}{*}{$\begin{array}{c}\text { Land- } \\
\text { surface } \\
\text { altitude } \\
\text { (feet above } \\
\text { sea level) }\end{array}$} & \multicolumn{4}{|c|}{ Water-level measurement } \\
\hline & & & & & \multirow[b]{2}{*}{ Date } & \multirow{2}{*}{$\begin{array}{c}\text { Feet } \\
\text { below } \\
\text { land } \\
\text { surface }\end{array}$} & \multirow[b]{2}{*}{ Status } & \multirow[b]{2}{*}{ Method } \\
\hline & $\begin{array}{l}\text { Local } \\
\text { Identification }\end{array}$ & $\begin{array}{l}\text { Standard } \\
\text { identificatlon }\end{array}$ & & & & & & \\
\hline \multirow[t]{8}{*}{153} & $\mathrm{~N} 20$ E53 22BDDDl & 393459115592501 & 320 & 6,015 & 04-05-66 & 137.33 & -- & $\mathrm{s}$ \\
\hline & & & & & $10-01-73$ & 135.6 & -- & $\mathrm{Z}$ \\
\hline & & & & & $11-03-75$ & 156.9 & -- & $\bar{z}$ \\
\hline & & & & & $03-01-76$ & 155.4 & -- & $\mathrm{Z}$ \\
\hline & & & & & $11-08-76$ & 161.1 & -- & $\mathrm{Z}$ \\
\hline & & & & & $11-14-77$ & 162.6 & -- & $\mathrm{Z}$ \\
\hline & & & & & 11-09-83 & 192.4 & -- & $\bar{Z}$ \\
\hline & & & & & $11-07-84$ & 192.0 & -- & $\mathrm{Z}$ \\
\hline \multirow[t]{12}{*}{153} & N20 E53 24DBBA1 & 393448115570201 & -- & 6,071 & $10-01-73$ & 193.9 & -- & $\mathrm{z}$ \\
\hline & & & & & $11-03-75$ & 185 & -- & $\mathrm{Z}$ \\
\hline & & & & & $03-01-76$ & 185 & -- & $\mathrm{Z}$ \\
\hline & & & & & $11-08-76$ & 200 & .. & $\mathrm{z}$ \\
\hline & & & & & $11-14-77$ & 200 & -- & $\mathrm{Z}$ \\
\hline & & & & & $11-08-78$ & 200 & -- & $\mathrm{Z}$ \\
\hline & & & & & $11-01-79$ & 200 & -- & $\mathrm{Z}$ \\
\hline & & & & & $11-01-80$ & 200 & -- & $\mathrm{Z}$ \\
\hline & & & & & $11-01-81$ & 200 & -- & $\mathrm{z}$ \\
\hline & & & & & $11-09-82$ & 200 & -- & $\mathrm{Z}$ \\
\hline & & & & & $11-08-83$ & 244.5 & -- & $\mathrm{Z}$ \\
\hline & & & & & $11-13-86$ & 247.4 & - & $\mathrm{z}$ \\
\hline \multirow[t]{5}{*}{153} & N20 E53 28ADDC1 & 393408116000301 & 225 & 6,024 & 04-04-66 & 154.14 & -- & $S$ \\
\hline & & & & & $11-09-83$ & 194 & -- & $\mathrm{Z}$ \\
\hline & & & & & $11-14-84$ & 195.5 & -- & $\mathrm{z}$ \\
\hline & & & & & $11-13-86$ & 196.9 & -- & $\mathrm{z}$ \\
\hline & & & & & $11-17-87$ & 200 & -- & $\mathrm{z}$ \\
\hline \multirow[t]{20}{*}{$153 N$} & N20 E53 28BCDD1 & 393401116003401 & 230 & 5,987 & $05-18-65$ & 128 & -- & -- \\
\hline & & & & & $04-04-66$ & 118.73 & -- & Z \\
\hline & & & & & $11-00-67$ & 125.0 & -- & $\mathrm{R}$ \\
\hline & & & & & $04-00-68$ & 121.4 & .. & $\mathrm{R}$ \\
\hline & & & & & $08-22-68$ & 123.4 & $\mathrm{~s}$ & $\mathrm{Z}$ \\
\hline & & & & & $04-00-69$ & 122.6 & -- & $\mathrm{R}$ \\
\hline & & & & & $09-08-69$ & 124.5 & $S$ & Z \\
\hline & & & & & $04-15-71$ & 126.0 & -- & Z \\
\hline & & & & & $11-17-71$ & 136.6 & -- & $\mathrm{Z}$ \\
\hline & & & & & $02-23-72$ & 135.6 & -- & Z \\
\hline & & & & & $12-05-72$ & 129.2 & -- & Z \\
\hline & & & & & $02-27-73$ & 129.1 & .. & $\bar{z}$ \\
\hline & & & & & $10-01-73$ & 131.8 & -- & Z \\
\hline & & & & & $03-12-74$ & 131.1 & -- & $\mathrm{Z}$ \\
\hline & & & & & $11-03-75$ & 139.6 & -- & $\mathrm{Z}$ \\
\hline & & & & & $03-01-76$ & 137.6 & -- & z \\
\hline & & & & & $11-08-76$ & 144.7 & -- & $\mathrm{Z}$ \\
\hline & & & & & $03-05-77$ & 143.5 & -- & $\mathrm{Z}$ \\
\hline & & & & & $11-14-77$ & 144.7 & -- & $\mathrm{Z}$ \\
\hline & & & & & $03-30-78$ & 142.8 & -- & $\bar{Z}$ \\
\hline
\end{tabular}


Appendix. Water-level measurements in selected wells, Diamond Valley, Nevada, 1960-90-Continued

\begin{tabular}{|c|c|c|c|c|c|c|c|c|}
\hline \multirow{2}{*}{\multicolumn{3}{|c|}{$\begin{array}{l}\text { U.S. Geological Survey } \\
\text { site designation }\end{array}$}} & \multirow{3}{*}{$\begin{array}{c}\text { Depth } \\
\text { of } \\
\text { well } \\
\text { (feet) }\end{array}$} & \multirow{3}{*}{$\begin{array}{c}\text { Land- } \\
\text { surface } \\
\text { altitude } \\
\text { (feet above } \\
\text { sea level) }\end{array}$} & \multicolumn{4}{|c|}{ Water-level measurement } \\
\hline & & & & & \multirow[b]{2}{*}{ Date } & \multirow{2}{*}{$\begin{array}{c}\text { Feet } \\
\text { below } \\
\text { land } \\
\text { surface }\end{array}$} & \multirow[b]{2}{*}{ Status } & \multirow[b]{2}{*}{ Methoc } \\
\hline & $\begin{array}{c}\text { Local } \\
\text { Identification }\end{array}$ & $\begin{array}{c}\text { Standard } \\
\text { identification }\end{array}$ & & & & & & \\
\hline \multirow{14}{*}{\multicolumn{3}{|c|}{153 N20 E53 28BCDD1 (Continued) }} & & & $11-08-78$ & 148.5 & -- & $\mathrm{Z}$ \\
\hline & & & & & $04-06-79$ & 145.7 & -- & $\mathbf{Z}$ \\
\hline & & & & & $11-01-79$ & 152.6 & -- & $\mathrm{Z}$ \\
\hline & & & & & $04-15-80$ & 150.3 & -- & $\mathrm{Z}$ \\
\hline & & & & & $11-01-80$ & 156.1 & -- & $\mathrm{Z}$ \\
\hline & & & & & $11-01-81$ & 160.4 & -- & $\mathrm{Z}$ \\
\hline & & & & & $11-09-82$ & 159.0 & -- & Z \\
\hline & & & & & $05-01-83$ & 161.4 & -- & Z \\
\hline & & & & & $11-09-83$ & 164.2 & -- & $\mathbf{Z}$ \\
\hline & & & & & $11-14-84$ & 162.6 & -- & $\mathrm{Z}$ \\
\hline & & & & & $11-15-86$ & 169.1 & -- & $\mathrm{Z}$ \\
\hline & & & & & $11-18-87$ & 178.3 & $\cdots$ & Z \\
\hline & & & & & $10-20-88$ & 187.4 & -- & Z \\
\hline & & & & & $10-23-89$ & 187.1 & -- & $\mathrm{Z}$ \\
\hline \multirow[t]{17}{*}{153} & N20 E53 29ADBB1 & 393410116011001 & 302 & 5,974 & $04-04-66$ & 105.97 & -- & $S$ \\
\hline & & & & & $10-01-73$ & 118.2 & -- & $\mathbf{Z}$ \\
\hline & & & & & $11-03-75$ & 126.6 & -- & $\mathrm{Z}$ \\
\hline & & & & & $03-01-76$ & 124.4 & -- & $\mathrm{Z}$ \\
\hline & & & & & $11-08-76$ & 128.6 & -- & $Z$ \\
\hline & & & & & $11-14-77$ & 132.1 & -. & $\mathbf{Z}$ \\
\hline & . & & & & $11-08-78$ & 135.5 & -- & Z \\
\hline & & & & & $11-01-79$ & 139.7 & -- & $\mathbf{Z}$ \\
\hline & & & & & $11-01-81$ & 146.0 & -- & Z \\
\hline & & & & & $05-01-83$ & 143.8 & -- & $\mathrm{Z}$ \\
\hline & & & & & $11-09-83$ & 148.0 & -- & $Z$ \\
\hline & & & & & $11-07-84$ & 145.9 & -- & Z \\
\hline & & & & & $12-04-85$ & 149.8 & -- & Z \\
\hline & & & & & $11-15-86$ & 150.8 & - & Z \\
\hline & & & & & $11-18-87$ & 159.7 & -- & $Z$ \\
\hline & & & & & $10-20-88$ & 168.1 & -- & $\mathrm{Z}$ \\
\hline & & & & & $10-23-89$ & 157.5 & -- & $\mathrm{Z}$ \\
\hline \multirow[t]{15}{*}{153} & N20 E53 30ABCCl & 393413116023001 & 155 & 5,984 & $12-00-60$ & 54.6 & -- & - \\
\hline & & & & & $11-00-63$ & 66.1 & -- & $\mathrm{R}$ \\
\hline & & & & & $11-00-64$ & 66.1 & -- & $\mathrm{R}$ \\
\hline & & & & & $04-00-65$ & 58.4 & -- & $\mathrm{R}$ \\
\hline & & & & & $11-00-65$ & 59.4 & -- & $\mathbf{R}$ \\
\hline & & & & & $04-04-66$ & 59.23 & -- & $Z$ \\
\hline & & & & & 09-08-69 & 73.0 & $S$ & $Z$ \\
\hline & & & & & $02-23-72$ & 61.7 & -. & $\mathrm{Z}$ \\
\hline & & & & & $12-05-72$ & 68.0 & -- & $Z$ \\
\hline & & & & & $02-27-73$ & 67.4 & -- & $\mathrm{Z}$ \\
\hline & & & & & $08-07-73$ & -. & $P$ & -- \\
\hline & & & & & $10-01-73$ & 70.5 & -- & $Z$ \\
\hline & & & & & $03-12-74$ & 85.1 & -- & $\mathrm{Z}$ \\
\hline & & & & & $07-26-74$ & 110 & -- & Z \\
\hline & & & & & $11-04-75$ & 86.1 & -- & $\mathrm{Z}$ \\
\hline
\end{tabular}


Appendix. Water-level measurements in selected wells, Diamond Valley, Nevada, 1960-90-Continued

\begin{tabular}{|c|c|c|c|c|c|c|c|c|}
\hline \multirow{2}{*}{\multicolumn{3}{|c|}{$\begin{array}{l}\text { U.S. Geological Survey } \\
\text { site designation }\end{array}$}} & \multirow{3}{*}{$\begin{array}{c}\text { Depth } \\
\text { of } \\
\text { well } \\
\text { (feet) }\end{array}$} & \multirow{3}{*}{$\begin{array}{c}\text { Land- } \\
\text { surface } \\
\text { altitude } \\
\text { (feet above } \\
\text { sea level) }\end{array}$} & \multicolumn{4}{|c|}{ Water-level measurement } \\
\hline & & & & & \multirow[b]{2}{*}{ Date } & \multirow{2}{*}{$\begin{array}{c}\text { Feet } \\
\text { below } \\
\text { land } \\
\text { surface }\end{array}$} & \multirow[b]{2}{*}{ Status } & \multirow[b]{2}{*}{ Method } \\
\hline & $\begin{array}{l}\text { Local } \\
\text { Identification }\end{array}$ & $\begin{array}{l}\text { Standard } \\
\text { identification }\end{array}$ & & & & & & \\
\hline \multirow{19}{*}{\multicolumn{2}{|c|}{$153 \mathrm{~N} 20 \mathrm{E} 5330 \mathrm{ABCC} 1$}} & (Continued) & & & $04-06-76$ & 80.8 & -- & $\mathrm{Z}$ \\
\hline & & & & & $11-08-76$ & 75.1 & -- & $\mathrm{Z}$ \\
\hline & & & & & $03-05-77$ & 65.0 & -- & $\mathrm{Z}$ \\
\hline & & & & & $11-15-77$ & 76.0 & -- & Z \\
\hline & & & & & $03-30-78$ & 73.4 & -- & $\mathrm{Z}$ \\
\hline & & & & & $11-10-78$ & 75.9 & -- & $z$ \\
\hline & & & & & $04-06-79$ & 74.1 & -- & $\mathrm{Z}$ \\
\hline & & & & & $11-09-79$ & 80.7 & -- & Z \\
\hline & & & & & $04-15-80$ & 75.5 & -- & $z$ \\
\hline & & & & & $11-19-80$ & 83.4 & -- & $\mathrm{Z}$ \\
\hline & & & & & $11-01-81$ & 91.8 & -- & $\mathrm{Z}$ \\
\hline & & & & & $11-09-82$ & 89.1 & -- & $\mathrm{Z}$ \\
\hline & & & & & $03-31-83$ & 90.1 & -- & Z \\
\hline & & & & & $11-09-83$ & 90.5 & -- & $\mathrm{Z}$ \\
\hline & & & & & $11-07-84$ & 88.1 & -- & $\mathrm{z}$ \\
\hline & & & & & $12-04-85$ & 88.5 & -- & $\mathrm{Z}$ \\
\hline & & & & & $11.05-86$ & 95.0 & -- & $\mathrm{Z}$ \\
\hline & & & & & $11-18-87$ & 94.0 & -- & $\mathrm{Z}$ \\
\hline & & & & & $10-20-88$ & 100.3 & -- & $\mathrm{z}$ \\
\hline \multirow{13}{*}{\multicolumn{2}{|c|}{153 N20 E53 30DCCC1 }} & 393343116023001 & 210 & 6,029 & $04-26-66$ & 94.19 & -- & $S$ \\
\hline & & & & & $10-01-73$ & 134.1 & -- & Z \\
\hline & & & & & $11-08-76$ & 138.1 & -- & $\mathrm{Z}$ \\
\hline & & & & & $11-14-77$ & 140.2 & -- & $\mathrm{Z}$ \\
\hline & & & & & $11-08-78$ & 142.9 & -- & $\mathrm{z}$ \\
\hline & & & & & $11-01-79$ & 148.6 & -- & $\mathrm{Z}$ \\
\hline & & & & & $11-01-80$ & 150.4 & -- & $\mathrm{Z}$ \\
\hline & & & & & $11-01-81$ & 157.2 & -- & $\mathrm{Z}$ \\
\hline & & & & & $12-05-85$ & 136.7 & -- & $\bar{z}$ \\
\hline & & & & & $11-05-86$ & 139.2 & .. & $\bar{Z}$ \\
\hline & & & & & $11-18-87$ & 131.1 & -- & $\mathrm{Z}$ \\
\hline & & & & & $10-20-88$ & 138.8 & -- & $\mathrm{Z}$ \\
\hline & & & & & $10-23-89$ & 140.4 & -- & $\mathrm{z}$ \\
\hline \multirow[t]{10}{*}{153} & N20 E53 31DAAC1 & 393310116020001 & 192 & 6,100 & $03-15-51$ & 158.55 & -- & -- \\
\hline & & & & & $09-11-51$ & 159.30 & -- & -- \\
\hline & & & & & $10-01-52$ & 157.11 & -- & -- \\
\hline & & & & & $12-11-52$ & 158.04 & -- & -- \\
\hline & & & & & $12-18-52$ & 157.89 & -- & -- \\
\hline & & & & & $12-27-52$ & 157.84 & -- & -- \\
\hline & & & & & $01-03-53$ & 157.81 & -- & -- \\
\hline & & & & & $01-06-53$ & 157.87 & -- & -- \\
\hline & & & & & $01-18-53$ & 157.84 & -- & -- \\
\hline & & & & & $01-25-53$ & 157.78 & -- & -- \\
\hline
\end{tabular}


Appendix. Water-level measurements in selected wells, Diamond Valley, Nevada, 1960-90-Continued

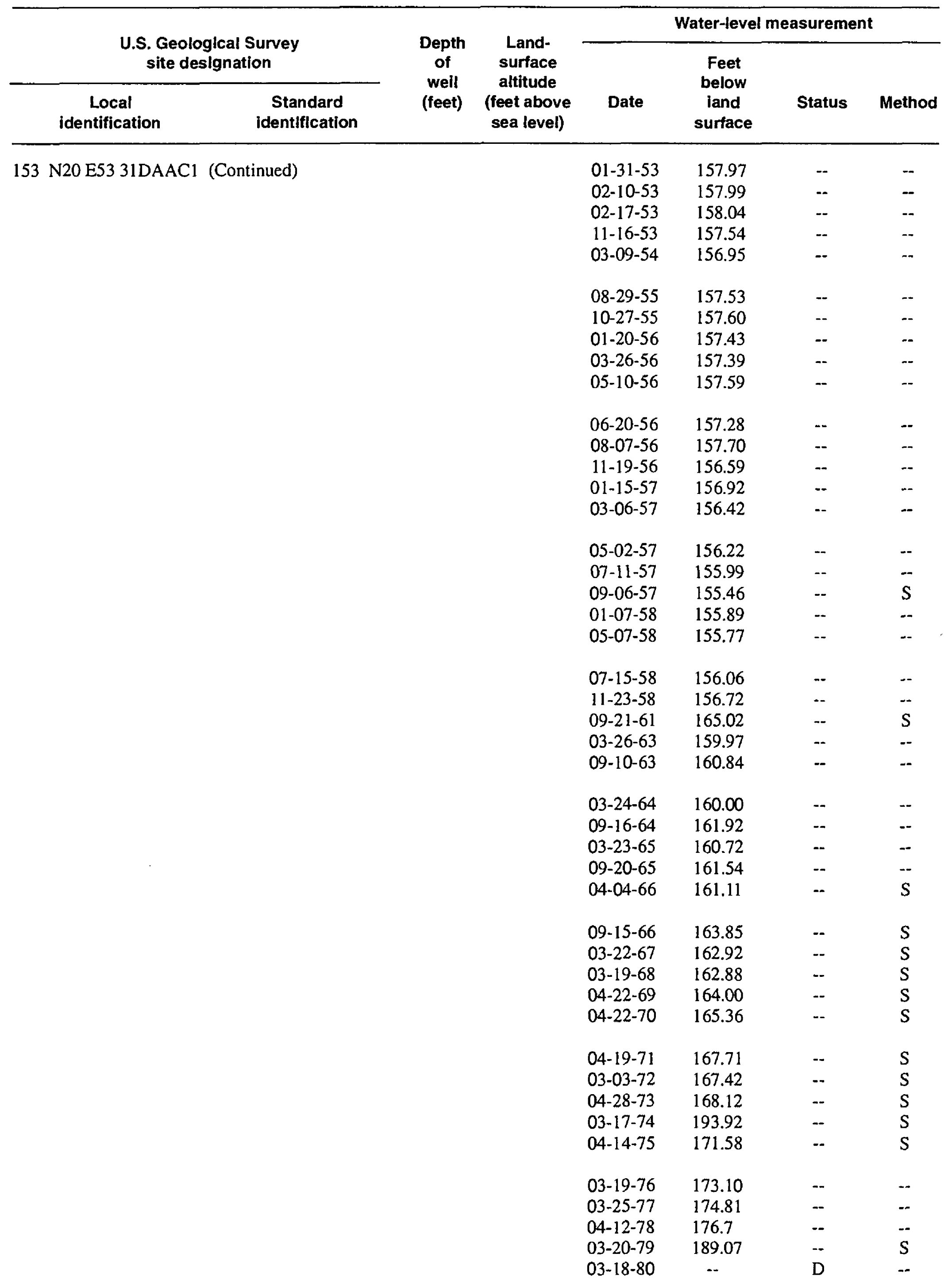


Appendix. Water-level measurements in selected wells, Diamond Valley, Nevada, 1960-90—Continued

\begin{tabular}{|c|c|c|c|c|c|c|c|c|}
\hline \multirow{2}{*}{\multicolumn{3}{|c|}{$\begin{array}{l}\text { U.S. Geological Survey } \\
\text { site designation }\end{array}$}} & \multirow{3}{*}{$\begin{array}{c}\text { Depth } \\
\text { of } \\
\text { well } \\
\text { (feet) }\end{array}$} & \multirow{3}{*}{$\begin{array}{c}\text { Land- } \\
\text { surface } \\
\text { altitude } \\
\text { (feet above } \\
\text { sea level) }\end{array}$} & \multicolumn{4}{|c|}{ Water-level measurement } \\
\hline & & & & & \multirow[b]{2}{*}{ Date } & \multirow{2}{*}{$\begin{array}{c}\text { Feet } \\
\text { below } \\
\text { land } \\
\text { surface }\end{array}$} & \multirow[b]{2}{*}{ Status } & \multirow[b]{2}{*}{ Method } \\
\hline & $\begin{array}{l}\text { Local } \\
\text { identificatlon }\end{array}$ & $\begin{array}{l}\text { Standard } \\
\text { identification }\end{array}$ & & & & & & \\
\hline \multirow[t]{3}{*}{153} & N20 E53 31DAACl & (Continued) & & & $03-17-81$ & -- & D & -- \\
\hline & & & & & $03-16-82$ & -- & D & -. \\
\hline & & & & & $03-25-85$ & -- & D & -- \\
\hline \multirow[t]{39}{*}{153} & N20 E53 32BBBA1 & 393332116015001 & 240 & 6,024 & 04-04-66 & 87.98 & -- & $\mathrm{Z}$ \\
\hline & & & & & $10-08-66$ & 98 & -- & -- \\
\hline & & & & & $11-00-67$ & 91.9 & -- & $\mathrm{R}$ \\
\hline & & & & & $04-00-68$ & 90.5 & -- & $\mathbf{R}$ \\
\hline & & & & & $08-21-68$ & 93.4 & $\mathrm{~s}$ & $\mathrm{Z}$ \\
\hline & & & & & $04-00-69$ & 91.6 & -- & $\mathbf{R}$ \\
\hline & & & & & $09-08-69$ & 94.8 & $\mathrm{~S}$ & $\mathrm{Z}$ \\
\hline & & & & & $04-14-71$ & 94.8 & -- & $\mathrm{Z}$ \\
\hline & & & & & $11-17-71$ & 98.2 & -- & $\mathrm{Z}$ \\
\hline & & & & & $02-23-72$ & 94.8 & -- & $\mathrm{Z}$ \\
\hline & & & & & $12-05-72$ & 99.7 & -- & $\mathrm{Z}$ \\
\hline & & & & & $08-07-73$ & 105.6 & $\mathrm{~s}$ & $\mathrm{Z}$ \\
\hline & & & & & $10-01-73$ & 98.9 & -- & $\mathrm{Z}$ \\
\hline & & & & & $03-12-74$ & 103.1 & -- & $\mathrm{Z}$ \\
\hline & & & & & $11-03-75$ & 102.8 & -- & $\mathrm{Z}$ \\
\hline & & & & & $11-08-76$ & 104.8 & -- & $\mathrm{Z}$ \\
\hline & & & & & $03-05-77$ & 101.7 & -- & $\mathrm{Z}$ \\
\hline & & & & & $11-14-77$ & 107.7 & - & $\mathrm{Z}$ \\
\hline & & & & & $03-30-78$ & 102.4 & -- & $\mathrm{Z}$ \\
\hline & & & & & $11-08-78$ & 111.6 & -- & $\mathrm{Z}$ \\
\hline & & & & & 04-06-79 & $108.8^{\circ}$ & -- & $\mathrm{Z}$ \\
\hline & & & & & $11-01-79$ & 111.3 & -- & $\mathrm{Z}$ \\
\hline & & & & & $04-15-80$ & 104.1 & -- & $\mathrm{Z}$ \\
\hline & & & & & $11-01-80$ & 113.9 & -. & $\mathrm{Z}$ \\
\hline & & & & & $11-01-81$ & 119.1 & -- & $\mathrm{Z}$ \\
\hline & & & & & $11-12-82$ & 117.8 & -- & $\mathrm{Z}$ \\
\hline & & & & & $03-31-83$ & 113.7 & .- & $\mathrm{Z}$ \\
\hline & & & & & $11-09-83$ & 119.5 & -- & $\mathrm{Z}$ \\
\hline & & & & & $11-07-84$ & 116.6 & -- & $\mathrm{Z}$ \\
\hline & & & & & $12-04-85$ & 121.1 & -- & $\mathrm{Z}$ \\
\hline & & & & & $11-05-86$ & 120.8 & -- & $\mathrm{Z}$ \\
\hline & & & & & $11-18-87$ & 128.3 & -- & $\mathrm{Z}$ \\
\hline & & & & & $10-20-88$ & 133.3 & -- & $\mathrm{Z}$ \\
\hline & & & & & $10-23-89$ & 133.2 & -- & $\mathrm{Z}$ \\
\hline & & 393327116013601 & 218 & 6,052 & $04-04-66$ & 112.98 & -- & $\mathrm{S}$ \\
\hline & & & & & $10-01-73$ & 137.5 & -- & $\mathrm{Z}$ \\
\hline & & & & & $11-03-75$ & 130.2 & -- & $\mathrm{Z}$ \\
\hline & & & & & $11-01-81$ & 162.6 & -- & $\bar{Z}$ \\
\hline & & & & & $11-09-83$ & 161.0 & -- & $\mathrm{Z}$ \\
\hline
\end{tabular}


Appendix. Water-level measurements in selected wells, Diamond Valley, Nevada, 1960-90-Continued

\begin{tabular}{|c|c|c|c|c|c|c|c|c|}
\hline \multirow{2}{*}{\multicolumn{3}{|c|}{$\begin{array}{l}\text { U.S. Geological Survey } \\
\text { site desIgnation }\end{array}$}} & \multirow{3}{*}{$\begin{array}{c}\text { Depth } \\
\text { of } \\
\text { well } \\
\text { (feet) }\end{array}$} & \multirow{3}{*}{$\begin{array}{c}\text { Land- } \\
\text { surface } \\
\text { altitude } \\
\text { (feet above } \\
\text { sea level) }\end{array}$} & \multicolumn{4}{|c|}{ Water-level measurement } \\
\hline & & & & & \multirow[b]{2}{*}{ Date } & \multirow{2}{*}{$\begin{array}{c}\text { Feet } \\
\text { below } \\
\text { land } \\
\text { surface }\end{array}$} & \multirow[b]{2}{*}{ Status } & \multirow[b]{2}{*}{ Method } \\
\hline & $\begin{array}{c}\text { Local } \\
\text { identificatlon }\end{array}$ & $\begin{array}{c}\text { Standard } \\
\text { identiflcation }\end{array}$ & & & & & & \\
\hline \multirow{6}{*}{\multicolumn{3}{|c|}{153 N20 E53 32BDCC1 (Continued) }} & & & $11-07-84$ & 155.2 & -- & $\mathrm{Z}$ \\
\hline & & & & & $12-04-85$ & 160.4 & -- & $\mathrm{Z}$ \\
\hline & & & & & $11-05-86$ & 167.0 & -. & $\mathrm{z}$ \\
\hline & & & & & $11-18-87$ & 170.8 & -- & $\mathrm{Z}$ \\
\hline & & & & & $10-20-88$ & 175.6 & -- & $\mathrm{Z}$ \\
\hline & & & & & $10-23-89$ & 163.3 & -- & $\mathrm{Z}$ \\
\hline \multirow[t]{14}{*}{153} & N20 E53 32CCAA1 & 393310116013901 & 255 & 6,062 & $04-04-66$ & 123.99 & -- & $\mathrm{s}$ \\
\hline & & & & & $10-01-73$ & 149.2 & -- & $\mathrm{Z}$ \\
\hline & & & & & $11-03-75$ & 138.7 & -- & $\mathrm{Z}$ \\
\hline & & & & & $03-01-76$ & 146.1 & -- & $\mathrm{Z}$ \\
\hline & & & & & $11-08-76$ & 140.0 & -- & $\mathrm{Z}$ \\
\hline & & & & & $11-14-77$ & 147.7 & -- & $\mathrm{Z}$ \\
\hline & & & & & $11-08-78$ & 154.8 & -- & $\mathrm{Z}$ \\
\hline & & & & & $11-01-79$ & 162.8 & -- & $\mathrm{Z}$ \\
\hline & & & & & $11-01-81$ & 174.1 & -- & $\mathrm{Z}$ \\
\hline & & & & & $11-09-83$ & 172.3 & -- & $\vec{Z}$ \\
\hline & & & & & $11-07-84$ & 170.4 & -- & $\mathrm{Z}$ \\
\hline & & & & & $12-04-85$ & 174.7 & -- & $\mathrm{z}$ \\
\hline & & & & & $11-05-86$ & 175.2 & -- & $\mathrm{Z}$ \\
\hline & & & & & $10-23-89$ & 156.1 & -- & $\mathrm{Z}$ \\
\hline \multirow[t]{7}{*}{153} & N20 E54 19BBCC1 & 393525115562001 & -. & 6,043 & $04-23-65$ & 172.19 & -- & $s$ \\
\hline & & & & & $11-01-80$ & 200 & -- & $\mathrm{Z}$ \\
\hline & & & & & $11-01-81$ & 200 & -- & $\mathrm{Z}$ \\
\hline & & & & & $11-09-82$ & 200 & -- & $\mathrm{Z}$ \\
\hline & & & & & $11-08-83$ & 211.0 & -- & $\mathrm{Z}$ \\
\hline & & & & & $11-14-84$ & 210.0 & -- & $\mathrm{Z}$ \\
\hline & & & & & $11-13-86$ & 214.6 & -- & $\mathrm{Z}$ \\
\hline \multirow[t]{12}{*}{153} & N21 E53 01BCAA1 & 394248115572701 & 210 & 5,884 & $04-06-66$ & 36.57 & -- & $\mathrm{s}$ \\
\hline & & & & & $11-01-80$ & 63.2 & -- & $\mathrm{Z}$ \\
\hline & & & & & $11-01-81$ & 68.9 & -- & $\mathrm{Z}$ \\
\hline & & & & & $11-09-82$ & 68.2 & -- & $\mathrm{Z}$ \\
\hline & & & & & $11-10-83$ & 68.5 & -- & $\mathrm{Z}$ \\
\hline & & & & & $11-09-84$ & 69.9 & -- & $\mathrm{Z}$ \\
\hline & & & & & $12-03-85$ & 74.4 & .. & $\mathrm{z}$ \\
\hline & & & & & $11-12-86$ & 74.6 & -- & $\mathrm{Z}$ \\
\hline & & & & & $11-17-87$ & 75.4 & -- & $\mathrm{Z}$ \\
\hline & & & & & $10-19-88$ & 81.9 & -- & $\mathrm{Z}$ \\
\hline & & & & & $11-02-89$ & 84.1 & -- & $\mathrm{Z}$ \\
\hline & & & & & $09-14-90$ & -- & $P$ & -- \\
\hline \multirow[t]{5}{*}{153} & N21 E53 01CDCC1 & 394232115572701 & 182 & 5,887 & $02-03-61$ & 33 & -- & -. \\
\hline & & & & & $04-00-65$ & 38.3 & -- & $\mathrm{Z}$ \\
\hline & & & & & $04-16-66$ & 37.98 & -- & $\mathrm{Z}$ \\
\hline & & & & & $11-00-66$ & 38.0 & -- & $\mathrm{Z}$ \\
\hline & & & & & $09-10-69$ & 54.0 & -- & $\mathrm{Z}$ \\
\hline
\end{tabular}


Appendix. Water-level measurements in selected wells, Diamond Valley, Nevada, 1960-90-Continued

\begin{tabular}{|c|c|c|c|c|c|c|c|c|}
\hline \multirow{2}{*}{\multicolumn{3}{|c|}{$\begin{array}{l}\text { U.S. Geological Survey } \\
\text { site designation }\end{array}$}} & \multirow{3}{*}{$\begin{array}{c}\text { Depth } \\
\text { of } \\
\text { well } \\
\text { (feet) }\end{array}$} & \multirow{3}{*}{$\begin{array}{c}\text { Land- } \\
\text { surface } \\
\text { altitude } \\
\text { (feet above } \\
\text { sea level) }\end{array}$} & \multicolumn{4}{|c|}{ Water-level measurement } \\
\hline & & & & & \multirow[b]{2}{*}{ Date } & \multirow{2}{*}{$\begin{array}{c}\text { Feet } \\
\text { below } \\
\text { land } \\
\text { surface }\end{array}$} & \multirow[b]{2}{*}{ Status } & \multirow[b]{2}{*}{ Method } \\
\hline & $\begin{array}{l}\text { Local } \\
\text { identification }\end{array}$ & $\begin{array}{l}\text { Standard } \\
\text { identification }\end{array}$ & & & & & & \\
\hline \multirow{15}{*}{\multicolumn{2}{|c|}{153 N21 E53 01CDCC 1}} & ontinued) & & & $02-22-72$ & 39.4 & -- & $\mathrm{Z}$ \\
\hline & & & & & $12-04-72$ & 40.4 & -- & $\mathrm{Z}$ \\
\hline & & & & & $11-05-75$ & 56.7 & -- & $\mathrm{Z}$ \\
\hline & & & & & $11-08-76$ & 58.1 & -- & $\mathrm{Z}$ \\
\hline & & & & & $03-05-77$ & 53.0 & -- & $\mathrm{z}$ \\
\hline & & & & & $11-14-77$ & 70.2 & -- & $\mathrm{Z}$ \\
\hline & & & & & $03-28-78$ & 65.1 & -- & $\mathrm{Z}$ \\
\hline & & & & & $11-08-78$ & 66.5 & -- & $\mathrm{Z}$ \\
\hline & & & & & $04-15-79$ & 60.8 & -- & $\mathrm{Z}$ \\
\hline & & & & & $11-06-79$ & 71.4 & -- & $\mathrm{z}$ \\
\hline & & & & & $04-04-80$ & 64.5 & -- & $\mathrm{Z}$ \\
\hline & & & & & $11-20-80$ & 70.8 & -- & $\mathrm{Z}$ \\
\hline & & & & & $11-00-81$ & 75.3 & -- & $\mathrm{Z}$ \\
\hline & & & & & $04-05-82$ & 67.6 & -- & $\mathrm{Z}$ \\
\hline & & & & & $03-29-83$ & 72.2 & -- & $\mathrm{z}$ \\
\hline \multirow[t]{23}{*}{153} & N21 E53 02CCAA1 & 394232115584201 & 182 & 5,887 & $05-01-61$ & 35 & -- & $\mathrm{R}$ \\
\hline & & & & & $11-00-63$ & 38.0 & -- & $\mathrm{R}$ \\
\hline & & & & & $04-00-64$ & 41.7 & -- & $\mathbf{R}$ \\
\hline & & & & & $04-00-65$ & 40.6 & -- & $\mathrm{R}$ \\
\hline & & & & & $11-00-65$ & 38.6 & -- & $\mathrm{R}$ \\
\hline & & & & & $11-00-66$ & 43.1 & -- & $\mathrm{R}$ \\
\hline & & & & & $11-00-67$ & 49.1 & -- & $\mathrm{R}$ \\
\hline & & & & & $04-00-68$ & 40.1 & -- & $\mathrm{R}$ \\
\hline & & & & & $08-26-68$ & 47.2 & -- & $\mathrm{Z}$ \\
\hline & & & & & $04-24-69$ & 41.9 & -- & $\mathrm{Z}$ \\
\hline & & & & & $09-10-69$ & 54.5 & -- & $\mathrm{z}$ \\
\hline & & & & & $11-19-71$ & 53.3 & -- & $\mathrm{Z}$ \\
\hline & & & & & $02-22-72$ & 40.9 & -- & $\mathrm{Z}$ \\
\hline & & & & & $12-04-72$ & -- & $\mathrm{P}$ & -- \\
\hline & & & & & $02-27-73$ & 36.8 & -- & $\mathrm{z}$ \\
\hline & & & & & $11-10-83$ & 69.6 & -- & $\mathrm{Z}$ \\
\hline & & & & & $11-09-84$ & 71.6 & -. & $\mathrm{Z}$ \\
\hline & & & & & $12-03-85$ & 73.6 & -- & $\mathrm{Z}$ \\
\hline & & & & & $11-12-86$ & 77.1 & -- & $\mathrm{Z}$ \\
\hline & & & & & $11-17-87$ & 78.0 & - & $\mathrm{z}$ \\
\hline & & & & & $10-19-88$ & 84.6 & -- & $\mathrm{Z}$ \\
\hline & & & & & $11-02-89$ & 84.8 & -- & $\mathrm{Z}$ \\
\hline & & & & & $09-14-90$ & -- & $P$ & -- \\
\hline \multirow[t]{5}{*}{153} & N21 E53 03BBDD2 & 394310115594702 & 198 & 5,882 & $10-19-67$ & 39 & -- & -- \\
\hline & & & & & $08-26-68$ & 45.2 & -- & $\mathrm{Z}$ \\
\hline & & & & & $04-24-69$ & 39.7 & -- & $\mathrm{Z}$ \\
\hline & & & & & $09-10-69$ & 49.7 & -- & $\mathrm{Z}$ \\
\hline & & & & & $11-19-71$ & 51.1 & -- & $\mathrm{Z}$ \\
\hline
\end{tabular}


Appendix. Water-level measurements in selected wells, Diamond Valley, Nevada, 1960-90-Continued

\begin{tabular}{|c|c|c|c|c|c|c|c|c|}
\hline \multirow{2}{*}{\multicolumn{3}{|c|}{$\begin{array}{l}\text { U.S. GeologIcal Survey } \\
\text { site designation }\end{array}$}} & \multirow{3}{*}{$\begin{array}{c}\text { Depth } \\
\text { of } \\
\text { well } \\
\text { (feet) }\end{array}$} & \multirow{3}{*}{$\begin{array}{l}\text { Land- } \\
\text { surface } \\
\text { altitude } \\
\text { (feet above } \\
\text { sea level) }\end{array}$} & \multicolumn{4}{|c|}{ Water-Jevel measurement } \\
\hline & & & & & \multirow[b]{2}{*}{ Date } & \multirow{2}{*}{$\begin{array}{c}\text { Feet } \\
\text { below } \\
\text { land } \\
\text { surface }\end{array}$} & \multirow[b]{2}{*}{ Status } & \multirow[b]{2}{*}{ Method } \\
\hline & $\begin{array}{c}\text { Local } \\
\text { identiflcation }\end{array}$ & $\begin{array}{l}\text { Standard } \\
\text { identification }\end{array}$ & & & & & & \\
\hline \multirow{18}{*}{\multicolumn{3}{|c|}{153 N21 E53 03BBDD2 (Continued) }} & & & $12-04-72$ & 46.5 & -- & $\mathrm{Z}$ \\
\hline & & & & & $02-27-73$ & 46.4 & -- & $\mathrm{Z}$ \\
\hline & & & & & $07-25-73$ & -- & $\mathbf{P}$ & -- \\
\hline & & & & & $03-11-74$ & 46.3 & -- & $\mathrm{Z}$ \\
\hline & & & & & $11-05-75$ & 52.6 & -. & $\mathrm{Z}$ \\
\hline & & & & & $04-07-76$ & 51.1 & -- & $\mathrm{Z}$ \\
\hline & & & & & $11-08-76$ & 55.0 & -- & $\mathrm{Z}$ \\
\hline & & & & & $03-05-77$ & 52.3 & -- & $\mathrm{Z}$ \\
\hline & & & & & $11-14-77$ & 59.0 & -- & Z \\
\hline & & & & & $03-28-78$ & 57.9 & -- & $\mathrm{Z}$ \\
\hline & & & & & $11-08-78$ & 60.0 & -- & $\mathrm{Z}$ \\
\hline & & & & & $04-05-79$ & 58.2 & -- & $\mathrm{Z}$ \\
\hline & & & & & $11-06-79$ & 68.0 & -- & $\mathrm{Z}$ \\
\hline & & & & & $04-04-80$ & 58.1 & -- & $\mathrm{Z}$ \\
\hline & & & & & $11-18-80$ & 68.9 & -- & $\mathrm{Z}$ \\
\hline & & & & & $11-00-81$ & 76.8 & -- & $\mathrm{R}$ \\
\hline & & & & & $04-05-82$ & 70.6 & -- & $\mathrm{Z}$ \\
\hline & & & & & $03-29-83$ & 67.8 & -- & $\mathrm{Z}$ \\
\hline \multirow{25}{*}{\multicolumn{2}{|c|}{153 N21 E53 03CDBB1 }} & 394230115594401 & 182 & 5,887 & $11-00-61$ & 37.8 & -- & $\mathrm{Z}$ \\
\hline & & & & & $03-00-66$ & 40.0 & .- & $\mathrm{Z}$ \\
\hline & & & & & $04-06-66$ & 40.00 & -- & $\mathrm{s}$ \\
\hline & & & & & $11-00-67$ & 48.6 & -- & $\mathrm{Z}$ \\
\hline & & & & & $11-00-71$ & 57.5 & -- & $\mathrm{z}$ \\
\hline & & & & & $03-00-72$ & 46.8 & -- & $\mathrm{Z}$ \\
\hline & & & & & $11-00-72$ & 47.9 & -- & Z \\
\hline & & & & & $03-00-73$ & 47.2 & -- & Z \\
\hline & & & & & $11-08-76$ & 60.0 & -- & Z \\
\hline & & & & & $11-14-77$ & 67.0 & -- & Z \\
\hline & & & & & $03-00-78$ & 59.3 & -- & Z \\
\hline & & & & & $11-08-78$ & 64.5 & -- & Z \\
\hline & & & & & $03-00-79$ & 61.0 & -- & Z \\
\hline & & & & & $11-01-79$ & 68.8 & -. & Z \\
\hline & & & & & $11-01-80$ & 70.3 & -- & Z \\
\hline & & & & & $11-01-81$ & 76.5 & -- & $\mathrm{Z}$ \\
\hline & & & & & $03-00-82$ & 68.5 & -- & $\mathrm{Z}$ \\
\hline & & & & & $11-09-82$ & 75.9 & -- & $\mathrm{Z}$ \\
\hline & & & & & $11-07-83$ & 72.6 & -- & Z \\
\hline & & & & & $11-09-84$ & 76.1 & -- & $\mathrm{z}$ \\
\hline & & & & & $12-03-85$ & 75.0 & -- & $\mathrm{Z}$ \\
\hline & & & & & $11-12-86$ & 79.1 & -- & Z \\
\hline & & & & & $11-17-87$ & 80.5 & -- & Z \\
\hline & & & & & $10-19-88$ & 85.6 & -- & $\mathrm{Z}$ \\
\hline & & & & & $11-02-89$ & 83.5 & -- & $\mathrm{Z}$ \\
\hline
\end{tabular}


Appendix. Water-level measurements in selected wells, Diamond Valley, Nevada, 1960-90-Continued

\begin{tabular}{|c|c|c|c|c|c|c|c|c|}
\hline \multirow{2}{*}{\multicolumn{3}{|c|}{$\begin{array}{l}\text { U.S. Geological Survey } \\
\text { site designation }\end{array}$}} & \multirow{3}{*}{$\begin{array}{c}\text { Depth } \\
\text { of } \\
\text { well } \\
\text { (feet) }\end{array}$} & \multirow{3}{*}{$\begin{array}{c}\text { Land- } \\
\text { surface } \\
\text { altitude } \\
\text { (feet above } \\
\text { sea level) }\end{array}$} & \multicolumn{4}{|c|}{ Water-level measurement } \\
\hline & & & & & \multirow[b]{2}{*}{ Date } & \multirow{2}{*}{$\begin{array}{c}\text { Feet } \\
\text { below } \\
\text { land } \\
\text { surface }\end{array}$} & \multirow[b]{2}{*}{ Status } & \multirow[b]{2}{*}{ Method } \\
\hline & $\begin{array}{l}\text { Local } \\
\text { identification }\end{array}$ & $\begin{array}{l}\text { Standard } \\
\text { identification }\end{array}$ & & & & & & \\
\hline \multirow{23}{*}{\multicolumn{2}{|c|}{153 N21 E53 03DDBB 1}} & 394238115593301 & 182 & 5,884 & $11-00-61$ & 38.2 & -- & $\mathrm{Z}$ \\
\hline & & & & & $11-00-63$ & 39.8 & -- & $\mathrm{Z}$ \\
\hline & & & & & $03-00-64$ & 40.7 & - & $\mathrm{Z}$ \\
\hline & & & & & $03-00-65$ & 38.5 & -- & $\mathrm{Z}$ \\
\hline & & & & & $11-00-65$ & 40.7 & -- & $\mathrm{Z}$ \\
\hline & & & & & $03-00-66$ & 39.1 & -- & $\mathrm{z}$ \\
\hline & & & & & $04-06-66$ & 39.12 & -- & $\mathrm{s}$ \\
\hline & & & & & $03-00-67$ & 40.1 & -- & $\mathrm{Z}$ \\
\hline & & & & & $11-00-67$ & 47.8 & -- & Z \\
\hline & & & & & $11-00-71$ & 55.6 & -- & $\mathrm{Z}$ \\
\hline & & & & & $03-00-72$ & 45.6 & -- & $\mathrm{Z}$ \\
\hline & & & & & $11-00-72$ & 46.1 & -- & $\mathrm{Z}$ \\
\hline & & & & & $03-00-73$ & 45.7 & -- & $\mathrm{Z}$ \\
\hline & & & & & $03-00-74$ & 47.1 & -- & $\mathrm{Z}$ \\
\hline & & & & & $11-00-75$ & 56.0 & -- & $\mathrm{Z}$ \\
\hline & & & & & $11-00-76$ & 52.8 & -- & $\mathrm{Z}$ \\
\hline & & & & & $03-00-77$ & 52.6 & -- & $\mathrm{Z}$ \\
\hline & & & & & $11-00-77$ & 54.4 & -- & $\mathrm{Z}$ \\
\hline & & & & & $11-00-78$ & 60.3 & -- & $\mathrm{Z}$ \\
\hline & & & & & 03-00-79 & 55.9 & -- & $\mathrm{Z}$ \\
\hline & & & & & $11-00-79$ & 68.0 & -- & $\mathrm{Z}$ \\
\hline & & & & & $11-00-80$ & 69.3 & - & $\mathrm{Z}$ \\
\hline & & & & & $11-00-81$ & 77.2 & -- & $\mathrm{Z}$ \\
\hline \multirow[t]{20}{*}{$153 \mathrm{I}$} & N21 E53 04ADD1 & 394258116000401 & 182 & 5,883 & $05-03-61$ & 36 & - & -- \\
\hline & & & & & $11-00-63$ & 37.2 & -- & $\mathbf{R}$ \\
\hline & & & & & $04-00-64$ & 37.8 & -- & $\mathrm{R}$ \\
\hline & & & & & $11-00-64$ & 40.8 & -- & $\mathbf{R}$ \\
\hline & & & & & $04-00-65$ & 36.5 & -- & $\mathrm{R}$ \\
\hline & & & & & $11-00-65$ & 38.6 & -- & $\mathrm{R}$ \\
\hline & & & & & $04-06-66$ & 40.38 & -- & $\mathrm{Z}$ \\
\hline & & & & & $11-00-67$ & 47.8 & -. & $\mathbf{R}$ \\
\hline & & & & & $04-00-68$ & 44.8 & -- & $\mathrm{R}$ \\
\hline & & & & & $08-26-68$ & 45.5 & -- & $\mathrm{Z}$ \\
\hline & & & & & $04-24-69$ & 42.2 & -- & $\mathrm{Z}$ \\
\hline & & & & & $09-10-69$ & 49.4 & -- & $\mathrm{Z}$ \\
\hline & & & & & $04-15-71$ & 67.9 & -- & $\mathrm{Z}$ \\
\hline & & & & & $11-19-71$ & 58.4 & -- & Z \\
\hline & & & & & $02-22-72$ & 47.6 & -- & $\mathrm{Z}$ \\
\hline & & & & & $12-04-72$ & 46.5 & -. & $\mathrm{Z}$ \\
\hline & & & & & $02-27-73$ & 43.5 & -- & Z \\
\hline & & & & & $07-25-73$ & -- & $P$ & -- \\
\hline & & & & & $10-30-73$ & 51.1 & -- & Z \\
\hline & & & & & $03-11-74$ & 47.8 & -- & $\mathrm{Z}$ \\
\hline
\end{tabular}


Appendix. Water-level measurements in selected wells, Diamond Valley, Nevada, 1960-90-Continued

\begin{tabular}{|c|c|c|c|c|c|c|c|c|}
\hline & \multirow{2}{*}{\multicolumn{2}{|c|}{$\begin{array}{l}\text { U.S. Geological Survey } \\
\text { site designatlon }\end{array}$}} & \multirow{3}{*}{$\begin{array}{c}\text { Depth } \\
\text { of } \\
\text { well } \\
\text { (feet) }\end{array}$} & \multirow{3}{*}{$\begin{array}{c}\text { Land- } \\
\text { surface } \\
\text { altitude } \\
\text { (feet above } \\
\text { sea level) }\end{array}$} & \multicolumn{4}{|c|}{ Water-level measurement } \\
\hline & & & & & \multirow[b]{2}{*}{ Date } & \multirow{2}{*}{$\begin{array}{c}\text { Feet } \\
\text { below } \\
\text { land } \\
\text { surface }\end{array}$} & \multirow[b]{2}{*}{ Status } & \multirow[b]{2}{*}{ Methoo } \\
\hline & $\begin{array}{l}\text { Local } \\
\text { Identification }\end{array}$ & $\begin{array}{l}\text { Standard } \\
\text { identification }\end{array}$ & & & & & & \\
\hline \multirow{14}{*}{\multicolumn{2}{|c|}{153 N21 E53 04ADD1 (C }} & ntinued & & & $11-05-75$ & 52.9 & -. & $\mathrm{Z}$ \\
\hline & & & & & $04-07-76$ & 50.1 & - & $\mathrm{Z}$ \\
\hline & & & & & $11-08-76$ & 55.5 & -- & $\mathrm{Z}$ \\
\hline & & & & & $03-05-77$ & 58.5 & -. & $\mathrm{Z}$ \\
\hline & & & & & $11-14-77$ & 57.9 & $\div-$ & $\mathrm{Z}$ \\
\hline & & & & & $03-28-78$ & 54.5 & -- & $Z$ \\
\hline & & & & & $11-08-78$ & 60.6 & -- & $\mathrm{Z}$ \\
\hline & & & & & $04-05-79$ & 56.0 & -- & $\mathrm{Z}$ \\
\hline & & & & & $11-06-79$ & 64.9 & -- & $\mathrm{Z}$ \\
\hline & & & & & 04-04-80 & 61.1 & -- & $z$ \\
\hline & & & & & $11-18-80$ & 62.3 & -- & $\mathrm{Z}$ \\
\hline & & & & & $11-00-81$ & 70.5 & -- & $\mathbf{R}$ \\
\hline & & & & & $04-05-82$ & 69.0 & -- & $\mathrm{Z}$ \\
\hline & & & & & $03-29-83$ & 69.1 & -- & $z$ \\
\hline \multirow[t]{11}{*}{153} & N21 E53 04CDBB1 & 394228116004601 & 182 & 5,882 & $06-06-66$ & 38.03 & -- & -- \\
\hline & & & & & $11-01-80$ & 53.0 & -- & $Z$ \\
\hline & & & & & $11-01-81$ & 69.4 & -- & $\mathrm{Z}$ \\
\hline & & & & & $11-09-82$ & 72.4 & -- & $\mathrm{Z}$ \\
\hline & & & & & $11-07-83$ & 76.1 & -- & $\mathrm{Z}$ \\
\hline & & & & & $11-14-84$ & 79.2 & -- & $\mathrm{Z}$ \\
\hline & & & & & $11-12-86$ & 79.4 & -- & $\mathrm{Z}$ \\
\hline & & & & & $11-17-87$ & 81.1 & -- & Z. \\
\hline & & & & & $10-19-88$ & 83.7 & -- & $\mathrm{z}$ \\
\hline & & & & & $11-02-89$ & 83.2 & -- & $\mathrm{Z}$ \\
\hline & & & & & $08-28-90$ & -- & $\mathrm{P}$ & -- \\
\hline \multirow[t]{20}{*}{153} & N21 E53 04DDD1 & 394233116000401 & 188 & 5,884 & $07-21-63$ & 42 & -- & -- \\
\hline & & & & & $11-00-63$ & 39.6 & -- & $Z$ \\
\hline & & & & & $04-00-64$ & 40.0 & -- & $\mathrm{Z}$ \\
\hline & & & & & $04-00-65$ & 29.9 & -- & Z \\
\hline & & & & & $04-06-66$ & 40.35 & -- & $z$ \\
\hline & & & & & $11-00-67$ & 53.9 & -- & $\mathrm{Z}$ \\
\hline & & & & & $04-00-68$ & 43.7 & -- & $Z$ \\
\hline & & & & & $08-26-68$ & 48.1 & -- & Z \\
\hline & & & & & $04-24-69$ & 44.2 & -- & $\mathrm{Z}$ \\
\hline & & & & & $09-10-69$ & 50.7 & -- & $\mathrm{Z}$ \\
\hline & & & & & $11-19-71$ & 57.2 & -- & $\mathrm{Z}$ \\
\hline & & & & & $02-22-72$ & 48.5 & -- & $Z$ \\
\hline & & & & & $12-04-72$ & 48.1 & -- & Z \\
\hline & & & & & $02-27-73$ & 46.2 & -- & $\mathrm{Z}$ \\
\hline & & & & & $03-11-74$ & 48.5 & -- & $\mathrm{Z}$ \\
\hline & & & & & $11-05-75$ & 54.4 & -- & Z \\
\hline & & & & & $03-05-76$ & 53.6 & -- & Z \\
\hline & & & & & $04-07-76$ & 53.1 & -- & $\mathrm{Z}$ \\
\hline & & & & & $11-08-76$ & 57.0 & -- & $\mathrm{Z}$ \\
\hline & & & & & $11-00-77$ & 62.5 & -- & $\mathrm{Z}$ \\
\hline
\end{tabular}


Appendix. Water-level measurements in selected wells, Diamond Valley, Nevada, 1960-90-Continued

\begin{tabular}{|c|c|c|c|c|c|c|c|c|}
\hline \multirow{2}{*}{\multicolumn{3}{|c|}{$\begin{array}{l}\text { U.S. Geological Survey } \\
\text { site designation }\end{array}$}} & \multirow{3}{*}{$\begin{array}{c}\text { Depth } \\
\text { of } \\
\text { well } \\
\text { (feet) }\end{array}$} & \multirow{3}{*}{$\begin{array}{c}\text { Land- } \\
\text { surface } \\
\text { altitude } \\
\text { (feet above } \\
\text { sea level) }\end{array}$} & \multicolumn{4}{|c|}{ Water-level measurement } \\
\hline & & & & & \multirow[b]{2}{*}{ Date } & \multirow{2}{*}{$\begin{array}{l}\text { Feet } \\
\text { below } \\
\text { land } \\
\text { surtace }\end{array}$} & \multirow[b]{2}{*}{ Status } & \multirow[b]{2}{*}{ Method } \\
\hline & $\begin{array}{l}\text { Local } \\
\text { Identification }\end{array}$ & $\begin{array}{l}\text { Standard } \\
\text { identification }\end{array}$ & & & & & & \\
\hline \multirow{9}{*}{\multicolumn{2}{|c|}{153 N21 E53 04DDD1 }} & ntinued) & & & $03-28-78$ & 58.8 & -- & $\mathrm{z}$ \\
\hline & & & & & $11-08-78$ & 59.6 & -- & $\mathrm{Z}$ \\
\hline & & & & & $04-05-79$ & 58.2 & -- & $\mathrm{Z}$ \\
\hline & & & & & $11-06-79$ & 68.6 & -- & Z \\
\hline & & & & & $04-04-80$ & 64.5 & -- & $\mathrm{Z}$ \\
\hline & & & & & $11-18-80$ & 70.6 & -- & $\mathrm{Z}$ \\
\hline & & & & & $11-00-81$ & 70.0 & -- & $\mathrm{Z}$ \\
\hline & & & & & $04-05-82$ & 63.3 & -- & $\mathrm{Z}$ \\
\hline & & & & & 03-29-83 & 65.4 & -- & $\mathrm{z}$ \\
\hline \multirow[t]{30}{*}{153} & N21 E53 05CBBC1 & 394250116020001 & 42.0 & 5,880 & $03-15-51$ & 28.87 & -- & -- \\
\hline & & & & & $09-11-51$ & 28.92 & -- & -- \\
\hline & & & & & $10-01-52$ & 28.86 & -- & -- \\
\hline & & & & & $03-03-53$ & 28.76 & -- & -- \\
\hline & & & & & $09-15-53$ & 28.69 & -- & -- \\
\hline & & & & & 03-08-54 & 28.61 & -- & $\mathrm{s}$ \\
\hline & & & & & $09-16-54$ & 28.68 & -- & -- \\
\hline & & & & & $08-29-55$ & 28.96 & -- & -- \\
\hline & & & & & $03-26-56$ & 28.93 & -- & -- \\
\hline & & & & & $08-28-56$ & 29.00 & - & -- \\
\hline & & & & & $09-12-61$ & 30.89 & -- & -- \\
\hline & & & & & $09-22-61$ & 30.88 & -- & -- \\
\hline & & & & & $03-25-62$ & 30.96 & -- & -- \\
\hline & & & & & $10-14-62$ & 32.28 & -- & -- \\
\hline & & & & & $03-25-63$ & 32.60 & -- & -- \\
\hline & & & & & $09-10-63$ & 33.08 & -- & -- \\
\hline & & & & & $03-24-64$ & 33.90 & -- & -- \\
\hline & & & & & $09-16-64$ & 33.73 & -- & -- \\
\hline & & & & & $03-23-65$ & 33.70 & -- & -- \\
\hline & & & & & $09-22-65$ & 34.55 & -- & $S$ \\
\hline & & & & & $04-06-66$ & 34.51 & -- & $\mathrm{S}$ \\
\hline & & & & & $09-15-66$ & 35.91 & -- & $S$ \\
\hline & & & & & $03-23-67$ & 35.97 & -- & $\mathrm{S}$ \\
\hline & & & & & $03-20-68$ & 37.06 & - & $\mathrm{S}$ \\
\hline & & & & & $04-22-69$ & 38.42 & -- & $S$ \\
\hline & & & & & $04-22-70$ & 38.80 & -- & $\mathrm{S}$ \\
\hline & & & & & $04-20-71$ & 39.50 & -- & $S$ \\
\hline & & & & & $03-03-72$ & 40.77 & -- & $S$ \\
\hline & & & & & $04-28-73$ & 41.48 & -- & $S$ \\
\hline & & & & & $03-14-75$ & -- & D & -- \\
\hline \multirow[t]{5}{*}{1531} & N21 E53 O6CDBB1 & 394243116030601 & -- & 5,879 & $11-03-75$ & 56.9 & -- & $\mathrm{Z}$ \\
\hline & & & & & $03-01-76$ & 51.7 & -. & Z \\
\hline & & & & & $11-08-76$ & 56.2 & -- & Z \\
\hline & & & & & $11-14-77$ & 58.9 & -- & Z \\
\hline & & & & & $11-08-78$ & 60.8 & -- & Z \\
\hline
\end{tabular}


Appendix. Water-level measurements in selected wells, Diamond Valley, Nevada, 1960-90-Continued

\begin{tabular}{|c|c|c|c|c|c|c|c|}
\hline \multirow{2}{*}{\multicolumn{2}{|c|}{$\begin{array}{l}\text { U.S. Geological Survey } \\
\text { site deslgnation }\end{array}$}} & \multirow{3}{*}{$\begin{array}{c}\text { Depth } \\
\text { of } \\
\text { well } \\
\text { (feet) }\end{array}$} & \multirow{3}{*}{$\begin{array}{c}\text { Land- } \\
\text { surface } \\
\text { altitude } \\
\text { (feet above } \\
\text { sea level) }\end{array}$} & \multicolumn{4}{|c|}{ Water-level measurement } \\
\hline & & & & \multirow[b]{2}{*}{ Date } & \multirow{2}{*}{$\begin{array}{c}\text { Feet } \\
\text { below } \\
\text { land } \\
\text { surface }\end{array}$} & \multirow[b]{2}{*}{ Status } & \multirow[b]{2}{*}{ Method } \\
\hline $\begin{array}{l}\text { Local } \\
\text { Identification }\end{array}$ & $\begin{array}{l}\text { Standard } \\
\text { Identificatlon }\end{array}$ & & & & & & \\
\hline \multirow[t]{12}{*}{153 N21 E53 06CDBB1 } & & & & $11-01-79$ & 64.7 & -- & $\mathrm{Z}$ \\
\hline & & & & $11-01-80$ & 66.4 & -- & $\mathrm{Z}$ \\
\hline & & & & $11-01-81$ & 70.6 & -- & $\mathrm{z}$ \\
\hline & & & & $11-09-82$ & 70.3 & -- & $z$ \\
\hline & & & & $11-07-83$ & 71.7 & -- & $\mathrm{Z}$ \\
\hline & & & & $11-14-84$ & 74.3 & -- & $\mathrm{Z}$ \\
\hline & & & & $12-03-85$ & 75.1 & -- & $\mathrm{Z}$ \\
\hline & & & & $11-12-86$ & 78.0 & -- & $\mathrm{Z}$ \\
\hline & & & & $11-17-87$ & 88.3 & -. & $\mathrm{Z}$ \\
\hline & & & & $10-19-88$ & 93.9 & -- & $z$ \\
\hline & & & & $11-02-89$ & 89.5 & .- & $\mathrm{Z}$ \\
\hline & & & & 08-27-90 & 126.9 & -- & $\mathrm{Z}$ \\
\hline \multirow[t]{14}{*}{153 N21 E53 06DCAA2 } & 394244116023501 & -- & 5,880 & $11-03-75$ & 50.2 & -- & $\mathrm{Z}$ \\
\hline & & & & $03-01-76$ & 47.5 & - & $\mathrm{Z}$ \\
\hline & & & & $11-08-76$ & 58.2 & -- & $\mathrm{Z}$ \\
\hline & & & & $11-14-77$ & 60.6 & -- & $\mathrm{Z}$ \\
\hline & & & & $11-08-78$ & 61.0 & -- & $\mathrm{Z}$ \\
\hline & & & & $11-01-79$ & 67.0 & -- & $\mathrm{z}$ \\
\hline & & & & $11-01-80$ & 64.1 & -- & $\mathrm{Z}$ \\
\hline & & & & $11-01-81$ & 71.3 & -- & $\mathrm{Z}$ \\
\hline & & & & $11-09-82$ & 65.3 & -- & $\mathrm{Z}$ \\
\hline & & & & $11-07-83$ & 65.4 & -- & $\mathrm{Z}$ \\
\hline & & & & $11-14-84$ & 72.1 & -- & $\mathrm{Z}$ \\
\hline & & & & $11-12-86$ & 73.3 & -- & $z$ \\
\hline & & & & $11-17-87$ & 79.1 & -- & $\mathrm{Z}$ \\
\hline & & & & $10-19-88$ & 84.8 & -- & $\mathrm{Z}$ \\
\hline \multirow[t]{20}{*}{153 N21 E53 08DCAA1 } & 394145116013401 & 192 & 5,893 & $11-00-61$ & 42.1 & -- & $\mathrm{Z}$ \\
\hline & & & & $11-00-63$ & 58.2 & -- & $\mathrm{Z}$ \\
\hline & & & & $03-00-66$ & 46.2 & -- & $z$ \\
\hline & & & & $04-06-66$ & 46.18 & -- & $S$ \\
\hline & & & & $11-00-67$ & 55.1 & -- & $\mathrm{Z}$ \\
\hline & & & & $03-00-68$ & 49.5 & -- & $\mathrm{Z}$ \\
\hline & & & & $11-00-68$ & 54.3 & .- & $\mathrm{Z}$ \\
\hline & & & & $03-00-69$ & 50.8 & -- & $\mathrm{Z}$ \\
\hline & & & & $11-00-69$ & 55.7 & -- & $\mathrm{Z}$ \\
\hline & & & & $11-00-71$ & 49.9 & -- & $\mathrm{Z}$ \\
\hline & & & & $03-00-72$ & 54.4 & -- & $\mathrm{Z}$ \\
\hline & & & & $11-00-72$ & 56.9 & -- & $\mathrm{Z}$ \\
\hline & & & & $03-00-74$ & 51.0 & -- & $\mathrm{Z}$ \\
\hline & & & & $11-08-76$ & 69.4 & -- & $\mathrm{Z}$ \\
\hline & & & & $11-08-78$ & 71.9 & -- & $z$ \\
\hline & & & & $03-00-79$ & 59.6 & .- & $\mathrm{Z}$ \\
\hline & & & & $11-01-80$ & 78.9 & -- & $\mathrm{Z}$ \\
\hline & & & & $11-01-81$ & 88.1 & -- & $\mathrm{Z}$ \\
\hline & & & & $03-00-82$ & 85.9 & -- & $\mathrm{Z}$ \\
\hline & & & & $11-09-82$ & 86.1 & -- & $Z$ \\
\hline
\end{tabular}


Appendix. Water-level measurements in selected wells, Diamond Valley, Nevada, 1960-90-Continued

\begin{tabular}{|c|c|c|c|c|c|c|c|c|}
\hline \multirow{2}{*}{\multicolumn{3}{|c|}{$\begin{array}{l}\text { U.S. Geological Survey } \\
\text { site designation }\end{array}$}} & \multirow{3}{*}{$\begin{array}{c}\text { Depth } \\
\text { of } \\
\text { well } \\
\text { (feet) }\end{array}$} & \multirow{3}{*}{$\begin{array}{c}\text { Land- } \\
\text { surface } \\
\text { altitude } \\
\text { (feet above } \\
\text { sea level) }\end{array}$} & \multicolumn{4}{|c|}{ Water-level measurement } \\
\hline & & & & & \multirow[b]{2}{*}{ Date } & \multirow{2}{*}{$\begin{array}{c}\text { Feet } \\
\text { below } \\
\text { land } \\
\text { surface }\end{array}$} & \multirow[b]{2}{*}{ Status } & \multirow[b]{2}{*}{ Method } \\
\hline & $\begin{array}{l}\text { Local } \\
\text { identification }\end{array}$ & $\begin{array}{l}\text { Standard } \\
\text { identlfication }\end{array}$ & & & & & & \\
\hline \multirow[t]{6}{*}{153} & N21 E53 08DCAA1 & (Continued) & & & $11-14-84$ & 91.3 & -- & $\mathrm{Z}$ \\
\hline & & & & & $11-12-86$ & 99.1 & -- & $\mathrm{Z}$ \\
\hline & & & & & $11-17-87$ & 102.3 & -- & $\mathrm{Z}$ \\
\hline & & & & & $10-19-88$ & 109.1 & -- & $\mathrm{Z}$ \\
\hline & & & & & $10-24-89$ & 102.3 & -- & $\mathrm{Z}$ \\
\hline & & & & & $08-30-90$ & -- & $\mathbf{P}$ & -- \\
\hline \multirow[t]{30}{*}{153} & N21 E53 09BBDD1 & 394219116005401 & 183 & 5,886 & $07-20-64$ & 55.0 & -- & -- \\
\hline & & & & & $11-00-64$ & 39.0 & -- & $\mathrm{R}$ \\
\hline & & & & & $11-00-65$ & 38.5 & -- & $\mathrm{R}$ \\
\hline & & & & & $04-06-66$ & 37.53 & -- & $\mathrm{Z}$ \\
\hline & & & & & $04-00-67$ & 39.2 & -- & $\bar{R}$ \\
\hline & & & & & $11-00-67$ & 47.1 & -- & $\mathrm{R}$ \\
\hline & & & & & $04-00-68$ & 41.3 & -- & $\mathrm{R}$ \\
\hline & & & & & $08-26-68$ & 52.5 & -- & $\mathrm{Z}$ \\
\hline & & & & & $04-23-69$ & 41.2 & -- & $\mathrm{Z}$ \\
\hline & & & & & 09-10-69 & 48.1 & -- & $\mathrm{Z}$ \\
\hline & & & & & $11-18-71$ & 45.9 & -- & $\mathrm{z}$ \\
\hline & & & & & $02-22-72$ & 48.7 & -- & $\mathrm{Z}$ \\
\hline & & & & & $12-04-72$ & 50.0 & -- & $\mathrm{Z}$ \\
\hline & & & & & $07-23-73$ & -- & $P$ & -- \\
\hline & & & & & $10-30-73$ & 60.3 & -- & $\mathrm{Z}$ \\
\hline & & & & & $03-12-74$ & 51.9 & -- & $\mathrm{Z}$ \\
\hline & & & & & $11-05-75$ & 53.7 & -- & $\mathrm{Z}$ \\
\hline & & & & & $04-07-76$ & 54,8 & -- & Z \\
\hline & & & & & $11-08-76$ & 57.6 & - & $\mathrm{Z}$ \\
\hline & & & & & $03-05-77$ & 54.0 & -- & $\mathrm{z}$ \\
\hline & & & & & $11-14-77$ & 62.0 & -- & $\mathrm{Z}$ \\
\hline & & & & & $03-28-78$ & 56.9 & -- & $\mathrm{Z}$ \\
\hline & & & & & $11-08-78$ & 62.3 & -- & $Z$ \\
\hline & & & & & $04-05-79$ & 58.7 & -- & $\mathrm{Z}$ \\
\hline & & & & & $11-06-79$ & 71.6 & -. & $\mathrm{Z}$ \\
\hline & & & & & $04-04-80$ & 69.0 & -- & $\mathrm{Z}$ \\
\hline & & & & & $11-18-80$ & 70.8 & -- & $\mathrm{Z}$ \\
\hline & & & & & $11-00-81$ & 73.6 & -- & $\mathrm{R}$ \\
\hline & & & & & $04-06-82$ & 66.8 & -- & $\mathrm{Z}$ \\
\hline & & & & & $03-29-83$ & 75.8 & -- & $\mathrm{z}$ \\
\hline \multirow[t]{5}{*}{153} & N21 E53 09DBDD1 & 394149116003201 & 182 & 5,893 & $04-06-66$ & 43.70 & -- & $\mathrm{s}$ \\
\hline & & & & & $10-01-73$ & 47.8 & -- & $\mathrm{Z}$ \\
\hline & & & & & $11-03-75$ & 45.2 & -- & $\mathrm{Z}$ \\
\hline & & & & & $03-01-76$ & 42.6 & -- & $\mathrm{Z}$ \\
\hline & & & & & $11-08-76$ & 50.7 & -- & $\mathrm{Z}$ \\
\hline
\end{tabular}


Appendix. Water-level measurements in selected wells, Diamond Valley, Nevada, 1960-90-Continued

\begin{tabular}{|c|c|c|c|c|c|c|c|c|}
\hline \multirow{2}{*}{\multicolumn{3}{|c|}{$\begin{array}{l}\text { U.S. Geological Survey } \\
\text { site designation }\end{array}$}} & \multirow{3}{*}{$\begin{array}{c}\text { Depth } \\
\text { of } \\
\text { well } \\
\text { (feet) }\end{array}$} & \multirow{3}{*}{$\begin{array}{c}\text { Land- } \\
\text { surface } \\
\text { altitude } \\
\text { (feet above } \\
\text { sea level) }\end{array}$} & \multicolumn{4}{|c|}{ Water-level measurement } \\
\hline & & & & & \multirow[b]{2}{*}{ Date } & \multirow{2}{*}{$\begin{array}{c}\text { Feet } \\
\text { below } \\
\text { land } \\
\text { surface }\end{array}$} & \multirow[b]{2}{*}{ Status } & \multirow[b]{2}{*}{ Method } \\
\hline & $\begin{array}{l}\text { Local } \\
\text { identiflcation }\end{array}$ & $\begin{array}{l}\text { Standard } \\
\text { Identification }\end{array}$ & & & & & & \\
\hline \multirow[t]{6}{*}{153} & N21 E53 09DBDD1 & Continued) & & & $11-14-77$ & 54.8 & -- & $\mathrm{Z}$ \\
\hline & & & & & $11-08-78$ & 58.4 & -- & $\mathrm{Z}$ \\
\hline & & & & & $11-01-79$ & 66.9 & -- & $\mathrm{Z}$ \\
\hline & & & & & $11-01-80$ & 69.3 & -- & $\mathrm{Z}$ \\
\hline & & & & & $10-24-89$ & 99.6 & -- & $\mathrm{Z}$ \\
\hline & & & & & $08-29-90$ & -- & $\mathrm{P}$ & -. \\
\hline \multirow[t]{12}{*}{153} & N21 E53 10CCAA1 & 394151115594801 & 182 & 5,894 & $07-18-61$ & 52 & -- & -- \\
\hline & & & & & $11-00-63$ & 44.6 & -- & $\mathrm{R}$ \\
\hline & & & & & $04-00-65$ & 43.5 & -- & $\mathrm{R}$ \\
\hline & & & & & $04-06-66$ & 49.80 & -. & $\mathrm{z}$ \\
\hline & & & & & $11-00-67$ & 51.7 & -- & $\mathrm{R}$ \\
\hline & & & & & $04-00-68$ & 48.1 & -- & $\mathrm{R}$ \\
\hline & & & & & $08-26-68$ & 52.8 & -- & $\mathrm{Z}$ \\
\hline & & & & & $04-23-69$ & 47.9 & -- & $\mathrm{z}$ \\
\hline & & & & & $09-10-69$ & 54.2 & -- & $\mathrm{z}$ \\
\hline & & & & & $11-19-71$ & 52.2 & -- & $\mathrm{Z}$ \\
\hline & & & & & $07-23-73$ & $-\infty$ & $\mathbf{P}$ & -- \\
\hline & & & & & $10-30-73$ & 53.3 & -- & $\mathrm{z}$ \\
\hline \multirow{16}{*}{\multicolumn{2}{|c|}{153 N21 E53 10DCAA2 }} & 394151115591301 & -- & 5,893 & $10-01-73$ & - & $P$ & -- \\
\hline & & & & & $11-03-75$ & 57.9 & -- & $\mathrm{Z}$ \\
\hline & & & & & $03-01-76$ & 53.6 & -- & $\mathrm{Z}$ \\
\hline & & & & & $11-08-76$ & 63.2 & -- & $\mathrm{Z}$ \\
\hline & & & & & $11-14-77$ & 66.5 & -- & Z \\
\hline & & & & & $11-08-78$ & 69.2 & -- & $\mathrm{z}$ \\
\hline & & & & & $11-01-79$ & 75.1 & -- & $\mathrm{Z}$ \\
\hline & & & & & $11-01-80$ & 75.2 & -- & $\mathrm{Z}$ \\
\hline & & & & & $11-01-81$ & 83.3 & -- & $\mathrm{Z}$ \\
\hline & & & & & $11-10-82$ & 80.4 & -- & Z \\
\hline & & & & & $11-07-83$ & 83.4 & -- & Z \\
\hline & & & & & $11-09-84$ & 85.6 & -- & Z \\
\hline & & & & & $11-12-86$ & 92.1 & -- & Z \\
\hline & & & & & $10-19-88$ & 102.5 & -- & $\mathrm{Z}$ \\
\hline & & & & & $10-24-89$ & 106.3 & -- & $\mathrm{Z}$ \\
\hline & & & & & $09-18-90$ & -- & $P$ & -- \\
\hline \multirow{10}{*}{\multicolumn{2}{|c|}{153 N21 E53 11BCAA1 }} & 394218115584401 & 192 & 5,886 & $11-06-60$ & 36.0 & -- & -- \\
\hline & & & & & $11-00-63$ & 41.9 & -- & $\mathbf{R}$ \\
\hline & & & & & $09-10-69$ & 54.2 & - & Z \\
\hline & & & & & $04-20-71$ & -- & $P$ & -- \\
\hline & & & & & $12-04-72$ & 49.2 & -- & $\mathrm{z}$ \\
\hline & & & & & $03-12-73$ & 48.9 & -- & $\mathrm{z}$ \\
\hline & & & & & $07-24-73$ & 89.1 & $S$ & $\mathrm{Z}$ \\
\hline & & & & & $11-02-73$ & 58 & -- & $\mathrm{Z}$ \\
\hline & & & & & $11-05-75$ & 56.4 & -- & Z \\
\hline & & & & & $04-06-76$ & 56.1 & -- & Z \\
\hline
\end{tabular}


Appendix. Water-level measurements in selected wells, Diamond Valley, Nevada, 1960-90-Continued

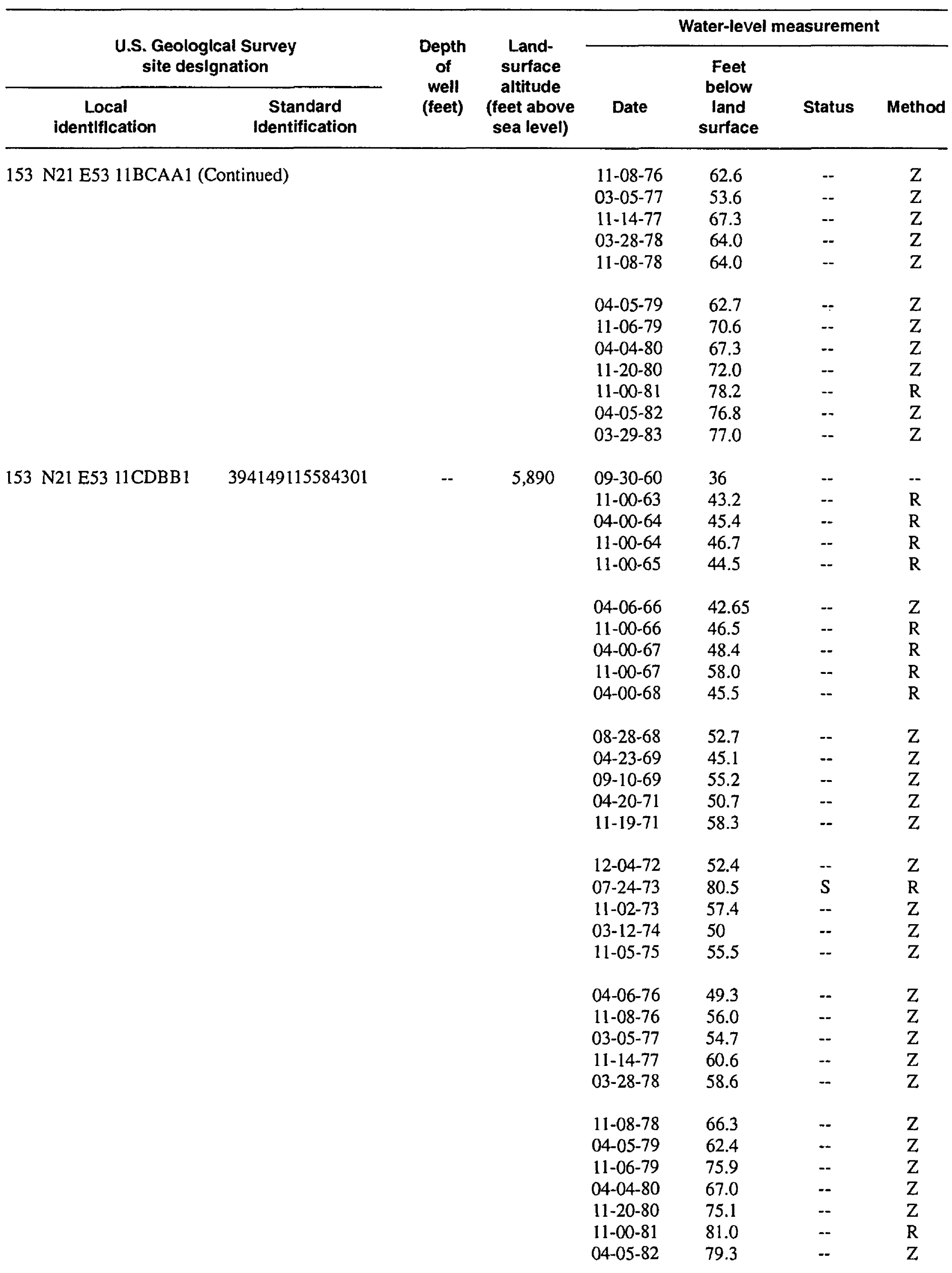


Appendix. Water-level measurements in selected wells, Diamond Valley, Nevada, 1960-90—Continued

\begin{tabular}{|c|c|c|c|c|c|c|c|c|}
\hline \multirow{2}{*}{\multicolumn{3}{|c|}{$\begin{array}{l}\text { U.S. Geological Survey } \\
\text { site designatlon }\end{array}$}} & \multirow{3}{*}{$\begin{array}{c}\text { Depth } \\
\text { of } \\
\text { well } \\
\text { (feet) }\end{array}$} & \multirow{3}{*}{$\begin{array}{c}\text { Land- } \\
\text { surface } \\
\text { altitude } \\
\text { (feet above } \\
\text { sea level) }\end{array}$} & \multicolumn{4}{|c|}{ Water-level measurement } \\
\hline & & & & & \multirow[b]{2}{*}{ Date } & \multirow{2}{*}{$\begin{array}{c}\text { Feet } \\
\text { below } \\
\text { land } \\
\text { surface }\end{array}$} & \multirow[b]{2}{*}{ Status } & \multirow[b]{2}{*}{ Method } \\
\hline & $\begin{array}{c}\text { Local } \\
\text { identification }\end{array}$ & $\begin{array}{c}\text { Standard } \\
\text { identification }\end{array}$ & & & & & & \\
\hline \multirow[t]{12}{*}{153} & N21 E53 11DACC1 & 394147115581001 & 183 & 5,893 & $04-06-66$ & 42.65 & -- & S \\
\hline & & & & & $11-14-77$ & 35.3 & -- & Z \\
\hline & & & & & $11-08-78$ & 67.0 & -- & $\mathrm{Z}$ \\
\hline & & & & & $11-01-79$ & 75.7 & -- & $\mathrm{Z}$ \\
\hline & & & & & $11-01-80$ & 74.7 & -- & $\mathrm{Z}$ \\
\hline & & & & & $11-01-81$ & 81.1 & -- & $\mathbf{Z}$ \\
\hline & & & & & $11-10-82$ & 85.1 & -- & $\mathrm{Z}$ \\
\hline & & & & & $11-10-83$ & 87.6 & -- & $\bar{Z}$ \\
\hline & & & & & $11-09-84$ & 87.3 & -- & $Z$ \\
\hline & & & & & $11-12-86$ & 86.5 & -- & $\mathrm{Z}$ \\
\hline & & & & & $10-24-89$ & 104.4 & -- & $\mathrm{Z}$ \\
\hline & & & & & $09-17-90$ & -- & $\mathbf{P}$ & -- \\
\hline \multirow{34}{*}{\multicolumn{2}{|c|}{153 N21 E53 12CCBC1 }} & 394144115574801 & 200 & 5,894 & $05-12-61$ & 44 & -- & -- \\
\hline & & & & & $11-00-63$ & 46.9 & -- & $\mathrm{R}$ \\
\hline & & & & & $04-00-64$ & 49.3 & -- & $\mathrm{R}$ \\
\hline & & & & & $11-00-64$ & 49.9 & - & $\mathrm{R}$ \\
\hline & & & & & $04-00-65$ & 42.6 & -- & $\mathrm{R}$ \\
\hline & & & & & $11-00-65$ & 49.4 & -- & $\mathrm{R}$ \\
\hline & & & & & $04-06-66$ & 44.28 & -- & $\mathrm{Z}$ \\
\hline & & & & & $04-00-67$ & 45.7 & -- & $\bar{R}$ \\
\hline & & & & & $11-00-67$ & 57.6 & -- & $\mathrm{R}$ \\
\hline & & & & & $04-00-68$ & 48.4 & -- & -- \\
\hline & & & & & $08-26-68$ & 55.1 & -- & $\mathrm{Z}$ \\
\hline & & & & & $04-23-69$ & 46.8 & -- & $\mathrm{Z}$ \\
\hline & & & & & $09-10-69$ & 57.0 & $S$ & $\mathrm{Z}$ \\
\hline & & & & & $04-20-71$ & 50.4 & -- & $\mathrm{Z}$ \\
\hline & & & & & $11-19-71$ & 59.3 & -- & $\mathrm{Z}$ \\
\hline & & & & & $02-22-72$ & 52.9 & -- & $\mathrm{Z}$ \\
\hline & & & & & $12-04-72$ & 53.6 & -- & Z \\
\hline & & & & & $07-24-73$ & 92.6 & $S$ & $\mathrm{Z}$ \\
\hline & & & & & $11-02-73$ & 60.5 & -- & $\mathrm{Z}$ \\
\hline & & & & & $03-12-74$ & 53.0 & -- & $\mathrm{Z}$ \\
\hline & & & & & $11-05-75$ & 60.1 & -- & $Z$ \\
\hline & & & & & $04-07-76$ & 58.3 & -- & $\mathrm{Z}$ \\
\hline & & & & & $11-08-76$ & 65.2 & -- & $\mathrm{Z}$ \\
\hline & & & & & $03-05-77$ & 58.0 & -- & $\mathrm{Z}$ \\
\hline & & & & & $11-14-77$ & 66.1 & -- & $Z$ \\
\hline & & & & & $03-28-78$ & 58.9 & -- & $\mathrm{Z}$ \\
\hline & & & & & $11-08-78$ & 66.8 & - & $Z$ \\
\hline & & & & & $04-05-79$ & 62.3 & -- & $\mathbf{Z}$ \\
\hline & & & & & $11-06-79$ & 76.9 & -- & $\mathrm{Z}$ \\
\hline & & & & & $04-04-80$ & 67.5 & -- & $\bar{Z}$ \\
\hline & & & & & $11-20-80$ & 65.4 & - & $\mathrm{Z}$ \\
\hline & & & & & $11-00-81$ & 81.5 & -- & $\mathrm{R}$ \\
\hline & & & & & $04-05-82$ & 77.6 & -- & $\mathrm{R}$ \\
\hline & & & & & $03-30-83$ & 74.5 & -- & $\mathrm{Z}$ \\
\hline
\end{tabular}


Appendix. Water-level measurements in selected wells, Diamond Valley, Nevada, 1960-90-Continued

\begin{tabular}{|c|c|c|c|c|c|c|c|}
\hline \multirow{2}{*}{\multicolumn{2}{|c|}{$\begin{array}{l}\text { U.S. Geological Survey } \\
\text { site desIgnatlon }\end{array}$}} & \multirow{3}{*}{$\begin{array}{c}\text { Depth } \\
\text { of } \\
\text { well } \\
\text { (feet) }\end{array}$} & \multirow{3}{*}{$\begin{array}{c}\text { Land- } \\
\text { surface } \\
\text { altitude } \\
\text { (feet above } \\
\text { sea level) }\end{array}$} & \multicolumn{4}{|c|}{ Water-level measurement } \\
\hline & & & & \multirow[b]{2}{*}{ Date } & \multirow{2}{*}{$\begin{array}{l}\text { Feet } \\
\text { below } \\
\text { land } \\
\text { surface }\end{array}$} & \multirow[b]{2}{*}{ Status } & \multirow[b]{2}{*}{ Method } \\
\hline $\begin{array}{c}\text { Local } \\
\text { identification }\end{array}$ & $\begin{array}{c}\text { Standard } \\
\text { identification }\end{array}$ & & & & & & \\
\hline \multirow[t]{18}{*}{153 N21 E53 12DCAA1 } & 394145115571701 & 192 & 5,889 & $04-06-66$ & 38.69 & -- & $S$ \\
\hline & & & & $10-01-73$ & 73.1 & -- & Z \\
\hline & & & & $11-03-75$ & 60.1 & -- & Z \\
\hline & & & & $03-01-76$ & 58.3 & - & $\mathbf{Z}$ \\
\hline & & & & $11-08-76$ & 57.6 & - & Z \\
\hline & & & & $11-14-77$ & 61.3 & -- & $\mathrm{Z}$ \\
\hline & & & & $11-08-78$ & 64.3 & -- & Z \\
\hline & & & & $11-01-79$ & 77.8 & -- & $\mathrm{Z}$ \\
\hline & & & & $11-01-80$ & 72.7 & -- & $\mathrm{Z}$ \\
\hline & & & & $11-01-81$ & 78.8 & -- & $\mathrm{Z}$ \\
\hline & & & & $11-10-82$ & 77.5 & -- & $\mathrm{Z}$ \\
\hline & & & & $11-10-83$ & 75.6 & -- & Z \\
\hline & & & & $11-09-84$ & 77.5 & -- & $\mathrm{Z}$ \\
\hline & & & & $11-12-86$ & 80.2 & -- & $\mathrm{Z}$ \\
\hline & & & & $11-17-87$ & 85.1 & -- & $\mathrm{Z}$ \\
\hline & & & & $10-19-88$ & 89.9 & -- & $\mathrm{Z}$ \\
\hline & & & & $10-24-89$ & 93.9 & -- & Z \\
\hline & & & & $09-17-90$ & -- & $\mathrm{P}$ & -- \\
\hline \multirow[t]{24}{*}{153 N21 E53 13BACC1 } & 394118115574601 & 182 & 5,896 & $11-00-61$ & 42.4 & -- & $\mathrm{Z}$ \\
\hline & & & & $11-00-64$ & 49.8 & -- & $Z$ \\
\hline & & & & $03-00-65$ & 48.5 & -- & $\mathrm{Z}$ \\
\hline & & & & $11-00-65$ & 49.7 & - & $\mathrm{Z}$ \\
\hline & & & & $03-00-66$ & 44.0 & - & $\mathrm{Z}$ \\
\hline & & & & $04-06-66$ & 43.99 & -- & $S$ \\
\hline & & & & $03-00-67$ & 46.1 & -- & $\mathrm{Z}$ \\
\hline & & & & $11-00-67$ & 58.0 & -- & $\mathrm{Z}$ \\
\hline & & & & $03-00-68$ & 50.8 & - & $\mathrm{Z}$ \\
\hline & & & & $11-00-68$ & 59.7 & -- & $\mathrm{Z}$ \\
\hline & & & & $03-00-69$ & 51.0 & -- & $\mathrm{Z}$ \\
\hline & & & & $11-00-69$ & 57.6 & -- & Z \\
\hline & & & & $11-00-71$ & 62.7 & -- & $\mathrm{Z}$ \\
\hline & & & & $11-00-72$ & 63.4 & -- & $\mathrm{Z}$ \\
\hline & & & & $03-00-73$ & 55.2 & $-\cdot$ & $\mathbf{Z}$ \\
\hline & & & & $03-00-74$ & 53.8 & -- & $\mathrm{Z}$ \\
\hline & & & & $11-00-75$ & 63.2 & -- & $\mathrm{Z}$ \\
\hline & & & & $03-00-76$ & 61.1 & -- & $\mathrm{Z}$ \\
\hline & & & & $11-00-76$ & 66.7 & -- & $\mathrm{Z}$ \\
\hline & & & & $03-00-77$ & 60.4 & -- & $\mathrm{Z}$ \\
\hline & & & & $11-00-77$ & 72.5 & -- & $\mathrm{Z}$ \\
\hline & & & & $11-00-78$ & 74.1 & -- & $\mathrm{Z}$ \\
\hline & & & & $11-00-80$ & 77.8 & -- & $\mathrm{Z}$ \\
\hline & & & & $11-00-81$ & 86.0 & $\cdots$ & $\mathrm{Z}$ \\
\hline
\end{tabular}


Appendix. Water-level measurements in selected wells, Diamond Valley, Nevada, 1960-90-Continued

\begin{tabular}{|c|c|c|c|c|c|c|c|c|}
\hline \multirow{2}{*}{\multicolumn{3}{|c|}{$\begin{array}{l}\text { U.S. Geological Survey } \\
\text { site designation }\end{array}$}} & \multirow{3}{*}{$\begin{array}{c}\text { Depth } \\
\text { of } \\
\text { well } \\
\text { (feet) }\end{array}$} & \multirow{3}{*}{$\begin{array}{c}\text { Land- } \\
\text { surface } \\
\text { altitude } \\
\text { (feet above } \\
\text { sea level) }\end{array}$} & \multicolumn{4}{|c|}{ Water-level measurement } \\
\hline & & & & & \multirow[b]{2}{*}{ Date } & \multirow{2}{*}{$\begin{array}{c}\text { Feet } \\
\text { below } \\
\text { land } \\
\text { surface }\end{array}$} & \multirow[b]{2}{*}{ Status } & \multirow[b]{2}{*}{ Method } \\
\hline & $\begin{array}{c}\text { Local } \\
\text { identification }\end{array}$ & $\begin{array}{c}\text { Standard } \\
\text { identification }\end{array}$ & & & & & & \\
\hline \multirow[t]{18}{*}{153} & N21 E53 14CACC1 & 394056115585001 & 180 & 5,899 & $04-06-66$ & 48.25 & -- & $S$ \\
\hline & & & & & $10-01-73$ & 64.5 & $\ldots$ & $\mathrm{Z}$ \\
\hline & & & & & $11-03-75$ & 66.7 & -- & $\mathrm{Z}$ \\
\hline & & & & & $11-08-76$ & 70.4 & -- & Z \\
\hline & & & & & $11-14-77$ & 73.5 & -- & $\mathrm{Z}$ \\
\hline & & & & & $11-08-78$ & 75.7 & -- & $\mathrm{Z}$ \\
\hline & & & & & $11-01-79$ & 85.6 & -- & $\mathrm{Z}$ \\
\hline & & & & & $11-01-80$ & 83.2 & -- & $\mathrm{Z}$ \\
\hline & & & & & $11-01-81$ & 92.8 & -- & $\mathrm{Z}$ \\
\hline & & & & & $11-10-82$ & 88.7 & -- & $\mathrm{Z}$ \\
\hline & & & & & $11-07-83$ & 89.2 & -- & $\mathrm{Z}$ \\
\hline & & & & & $11-09-84$ & 90.7 & $\cdots$ & $\mathrm{Z}$ \\
\hline & & & & & $12-04-85$ & 92.0 & -- & $\mathrm{Z}$ \\
\hline & & & & & $11-13-86$ & 93.2 & -- & $\mathrm{Z}$ \\
\hline & & & & & $11-18-87$ & 101.5 & -- & $\mathrm{Z}$ \\
\hline & & & & & $10-19-88$ & 108.9 & -- & $\mathrm{Z}$ \\
\hline & & & & & $10-24-89$ & 106.0 & - & $\mathrm{Z}$ \\
\hline & & & & & $09-18-90$ & -- & $\mathbf{P}$ & -- \\
\hline \multirow[t]{26}{*}{153} & N21 E53 15BACC1 & 394118115595501 & 182 & 5,898 & $11-00-61$ & 43.2 & -- & $Z$ \\
\hline & & & & & $11-00-64$ & 48.1 & -- & $\mathbf{Z}$ \\
\hline & & & & & $11-00-65$ & 47.7 & -- & $\mathrm{Z}$ \\
\hline & & & & & $03-00-66$ & 46.1 & -- & $\mathrm{Z}$ \\
\hline & & & & & $04-06-66$ & 46.10 & -- & $S$ \\
\hline & & & & & $03-00-67$ & 48.3 & -- & $\mathrm{Z}$ \\
\hline & & & & & $11-00-67$ & 57.9 & -- & Z \\
\hline & & & & & $03-00-68$ & 54.1 & -- & $\mathrm{Z}$ \\
\hline & & & & & $11-00-68$ & 56.7 & - & $Z$ \\
\hline & & & & & $03-00-69$ & 50.0 & -- & $\mathbf{Z}$ \\
\hline & & & & & $11-00-69$ & 59.1 & -- & $\mathrm{Z}$ \\
\hline & & & & & $11-00-71$ & 57.7 & -- & $\mathrm{Z}$ \\
\hline & & & & & $03-00-72$ & 55.5 & -- & $\mathrm{Z}$ \\
\hline & & & & & $11-00-72$ & 57.9 & -- &. $\mathrm{Z}$ \\
\hline & & & & & $11-00-73$ & 60.3 & -- & $\mathrm{Z}$ \\
\hline & & & & & $03-00-74$ & 62.6 & -- & $\mathrm{Z}$ \\
\hline & & & & & $11-00-75$ & 63.6 & -. & $\mathrm{Z}$ \\
\hline & & & & & $03-00-76$ & 59.4 & -- & $\mathrm{Z}$ \\
\hline & & & & & $11-00-76$ & 67.7 & -. & $\mathrm{Z}$ \\
\hline & & & & & $03-00-77$ & 61.3 & -- & $\mathrm{Z}$ \\
\hline & & & & & $11-00-77$ & 72.2 & -- & $\mathrm{Z}$ \\
\hline & & & & & $03-00-78$ & 68.0 &.- & $\mathrm{Z}$ \\
\hline & & & & & $11-00-78$ & 74.2 & -. & $\mathrm{Z}$ \\
\hline & & & & & $03-00-79$ & 69.8 & -- & $\mathrm{Z}$ \\
\hline & & & & & $11-00-79$ & 89.1 & -- & $\mathrm{Z}$ \\
\hline & & & & & $11-00-80$ & 90.7 & - & $\mathrm{Z}$ \\
\hline
\end{tabular}


Appendix. Water-level measurements in selected wells, Diamond Valley, Nevada, 1960-90-Continued

\begin{tabular}{|c|c|c|c|c|c|c|c|c|}
\hline \multirow{2}{*}{\multicolumn{3}{|c|}{$\begin{array}{l}\text { U.S. Geologlcal Survey } \\
\text { site designation }\end{array}$}} & \multirow{3}{*}{$\begin{array}{c}\text { Depth } \\
\text { of } \\
\text { well } \\
\text { (feet) }\end{array}$} & \multirow{3}{*}{$\begin{array}{l}\text { Land- } \\
\text { surface } \\
\text { altitude } \\
\text { (feet above } \\
\text { sea level) }\end{array}$} & \multicolumn{4}{|c|}{ Water-level measurement } \\
\hline & & & & & \multirow[b]{2}{*}{ Date } & \multirow{2}{*}{$\begin{array}{c}\text { Feet } \\
\text { below } \\
\text { land } \\
\text { surface }\end{array}$} & \multirow[b]{2}{*}{ Status } & \multirow[b]{2}{*}{ Method } \\
\hline & $\begin{array}{l}\text { Local } \\
\text { Identification }\end{array}$ & $\begin{array}{l}\text { Standard } \\
\text { identificatlon }\end{array}$ & & & & & & \\
\hline \multirow{18}{*}{\multicolumn{2}{|c|}{153 N21 E53 16CCAA2 }} & 394101116005701 & -- & 5,911 & $10-01-73$ & 75.4 & -- & $\mathrm{Z}$ \\
\hline & & & & & $11-03-75$ & 69.2 & -- & $\mathrm{Z}$ \\
\hline & & & & & $03-01-76$ & 67.3 & -. & $\mathrm{Z}$ \\
\hline & & & & & $11-08-76$ & 86.3 & -- & $\mathrm{Z}$ \\
\hline & & & & & $11-14-77$ & 89.1 & -- & $\mathrm{Z}$ \\
\hline & & & & & $11-08-78$ & 91.1 & -- & $\mathrm{Z}$ \\
\hline & & & & & $11-01-79$ & 104.3 & -- & Z \\
\hline & & & & & $11-01-80$ & 101.8 & -. & $\mathrm{Z}$ \\
\hline & & & & & $11-01-81$ & 113.5 & -- & Z \\
\hline & & & & & $11-10-82$ & 116.2 & -- & $\mathrm{Z}$ \\
\hline & & & & & $11-09-83$ & 119.1 & -- & $\mathrm{Z}$ \\
\hline & & & & & $11-08-84$ & 117.9 & -- & $\mathrm{Z}$ \\
\hline & & & & & $12-04-85$ & 124.2 & -- & $\mathrm{Z}$ \\
\hline & & & & & $11-13-86$ & 126.5 & -- & $\mathrm{Z}$ \\
\hline & & & & & $11-18-87$ & 134.6 & - & $\mathrm{Z}$ \\
\hline & & & & & $10-21-88$ & 135.0 & -- & $\mathrm{Z}$ \\
\hline & & & & & $11-29-89$ & 122.3 & -- & $\mathrm{Z}$ \\
\hline & & & & & $09-10-90$ & $\cdot-$ & $\mathbf{P}$ & -- \\
\hline \multirow{25}{*}{\multicolumn{2}{|c|}{153 N21 E53 17CDBB1 }} & 394104116020001 & 200 & 5,922 & $06-03-64$ & 56 & -- & -- \\
\hline & & & & & $04-00-65$ & 73.5 & -. & $\mathrm{R}$ \\
\hline & & & & & $11-00-65$ & 75.7 & .. & $\mathrm{R}$ \\
\hline & & & & & $04-06-66$ & 74.52 & -- & $\mathrm{Z}$ \\
\hline & & & & & $11-00-66$ & 78.4 & - & $\mathrm{R}$ \\
\hline & & & & & $04-00-67$ & 79.2 & -- & $\mathrm{R}$ \\
\hline & & & & & $11-00-67$ & 84.3 & .. & $\mathrm{R}$ \\
\hline & & & & & $04-00-68$ & 77.8 & -- & $\mathrm{R}$ \\
\hline & & & & & $08-23-68$ & 83.6 & $S$ & Z \\
\hline & & & & & $04-19-69$ & 79.2 & -- & $\mathrm{Z}$ \\
\hline & & & & & 09-09-69 & 82.4 & -- & Z \\
\hline & & & & & $04-20-71$ & 85.5 & -- & Z \\
\hline & & & & & $11-18-71$ & 83.9 & -- & -- \\
\hline & & & & & $02-23-72$ & 96.5 & -. & $\mathrm{Z}$ \\
\hline & & & & & $07-25-73$ & 102.9 & $S$ & $\mathrm{z}$ \\
\hline & & & & & $11-03-73$ & 92.6 & -- & Z \\
\hline & & & & & $03-11-74$ & 86.1 & -- & Z \\
\hline & & & & & $11-03-75$ & 94.3 & -- & $\mathrm{Z}$ \\
\hline & & & & & $04-05-76$ & 90.7 & -- & Z \\
\hline & & & & & $11-08-76$ & 100.0 & -- & $\mathrm{Z}$ \\
\hline & & & & & $03-05-77$ & 94.5 & -- & $\mathrm{Z}$ \\
\hline & & & & & $11-14-77$ & 103.3 & -. & Z \\
\hline & & & & & $03-28-78$ & 101.8 & -- & Z \\
\hline & & & & & $11-08-78$ & 102.9 & -. & Z \\
\hline & & & & & 04-09-79 & 91.6 & -- & $\mathrm{Z}$ \\
\hline
\end{tabular}


Appendix. Water-level measurements in selected wells, Diamond Valley, Nevada, 1960-90-Continued

\begin{tabular}{|c|c|c|c|c|c|c|c|c|}
\hline \multirow{2}{*}{\multicolumn{3}{|c|}{$\begin{array}{c}\text { U.S. Geologlcal Survey } \\
\text { site designatlon }\end{array}$}} & \multirow{3}{*}{$\begin{array}{c}\text { Depth } \\
\text { of } \\
\text { weil } \\
\text { (feet) }\end{array}$} & \multirow{3}{*}{$\begin{array}{l}\text { Land- } \\
\text { surface } \\
\text { altitude } \\
\text { (feet above } \\
\text { sea ievei) }\end{array}$} & \multicolumn{4}{|c|}{ Water-level measurement } \\
\hline & & & & & \multirow[b]{2}{*}{ Date } & \multirow{2}{*}{$\begin{array}{c}\text { Feet } \\
\text { below } \\
\text { land } \\
\text { surface }\end{array}$} & \multirow[b]{2}{*}{ Status } & \multirow[b]{2}{*}{ Method } \\
\hline & $\begin{array}{c}\text { Local } \\
\text { Identification }\end{array}$ & $\begin{array}{l}\text { Standard } \\
\text { Identitlcation }\end{array}$ & & & & & & \\
\hline \multirow{6}{*}{\multicolumn{3}{|c|}{153 N21 E53 17CDBB1 (Continued) }} & & & $11-06-79$ & 109.8 & -- & $\mathrm{Z}$ \\
\hline & & & & & 04-04-80 & 102.2 & -- & $\mathrm{Z}$ \\
\hline & & & & & $11-18-80$ & 110.2 & -- & $\mathrm{Z}$ \\
\hline & & & & & $11-00-81$ & 116.2 & -- & $\mathrm{R}$ \\
\hline & & & & & $04-06-82$ & 110.6 & -. & $\mathrm{z}$ \\
\hline & & & & & 03-29-83 & 114.9 & -- & $\mathrm{z}$ \\
\hline \multirow{16}{*}{\multicolumn{2}{|c|}{153 N21 E53 20AACCl }} & 394037116102101 & 196 & 5,926 & $03-00-61$ & 70.8 & -- & $\mathrm{Z}$ \\
\hline & & & & & $09-13-61$ & 72 & -- & $\mathrm{R}$ \\
\hline & & & & & $03-00-64$ & 75.9 & -- & $\mathrm{Z}$ \\
\hline & & & & & $09-15-64$ & 75.92 & -- & $\mathrm{S}$ \\
\hline & & & & & 03-00-65 & 75.9 & -- & $\mathrm{z}$ \\
\hline & & & & & $04-28-65$ & 73.5 & -- & $\mathrm{Z}$ \\
\hline & & & & & $10-18-65$ & 75.90 & -- & $S$ \\
\hline & & & & & $04-06-66$ & 74.73 & -- & $\mathrm{S}$ \\
\hline & & & & & $03-00-68$ & 102.0 & -- & $\mathrm{Z}$ \\
\hline & & & & & $03-00-69$ & 90.7 & -- & $\mathrm{Z}$ \\
\hline & & & & & $03-00-71$ & 94.1 & -- & $\mathrm{Z}$ \\
\hline & & & & & $03-00-75$ & 97.0 & -- & $\mathrm{Z}$ \\
\hline & & & & & $03-00-77$ & 109.6 & -- & $\mathrm{Z}$ \\
\hline & & & & & $03-00-78$ & 104.6 & -- & $\mathrm{Z}$ \\
\hline & & & & & $03-00-79$ & 110.8 & -- & $\mathrm{Z}$ \\
\hline & & & & & $03-00-80$ & 116.4 & -- & $\mathrm{Z}$ \\
\hline \multirow{14}{*}{\multicolumn{2}{|c|}{153 N21 E53 20DDBB1 }} & 394007116012801 & -- & 5,935 & $10-01-73$ & 107.5 & -. & $\mathrm{Z}$ \\
\hline & & & & & $11-03-75$ & 105.4 & -- & $\mathrm{Z}$ \\
\hline & & & & & $03-01-76$ & 103.1 & -. & $\mathrm{Z}$ \\
\hline & & & & & $11-14-77$ & 115.7 & -- & $\mathrm{Z}$ \\
\hline & & & & & $11-01-80$ & 125.2 & -- & $\mathrm{Z}$ \\
\hline & & & & & $11-01-81$ & 134.6 & -- & $\mathrm{Z}$ \\
\hline & & & & & $11-10-82$ & 133.1 & -- & $\mathrm{Z}$ \\
\hline & & & & & $11-09-83$ & 131.4 & -- & $\mathrm{Z}$ \\
\hline & & & & & $11-08-84$ & 130.3 & -- & $\mathrm{Z}$ \\
\hline & & & & & $12-04-85$ & 136.1 & -- & $\mathrm{Z}$ \\
\hline & & & & & $11-05-86$ & 140.3 & -- & $\mathrm{Z}$ \\
\hline & & & & & $11-18-87$ & 143.0 & - & $\mathrm{Z}$ \\
\hline & & & & & $10-21-88$ & 146.5 & -- & $\mathrm{Z}$ \\
\hline & & & & & $09-11-90$ & -- & $P$ & -- \\
\hline \multirow[t]{10}{*}{153} & N21 E53 21 BBDD1 & 394033116005201 & 190 & 5,918 & $08-31-61$ & 52 & - & -- \\
\hline & & & & & $11-00-63$ & 62.9 & - & $\mathrm{R}$ \\
\hline & & & & & $04-00-64$ & 62.3 & -- & $\mathbf{R}$ \\
\hline & & & & & $11-00-64$ & 65.2 & -- & $\mathbf{R}$ \\
\hline & & & & & $04-00-65$ & 64.7 & -- & $\mathrm{R}$ \\
\hline & & & & & $11-00-65$ & 65.1 & -. & $\mathbf{R}$ \\
\hline & & & & & $04-06-66$ & 63.55 & -- & $\mathrm{Z}$ \\
\hline & & & & & $04-00-67$ & 60.6 & -- & $\mathrm{R}$ \\
\hline & & & & & $11-00-67$ & 76.0 & 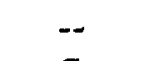 & $\mathrm{R}$ \\
\hline & & & & & $08-23-68$ & 83.2 & $S$ & $\mathrm{Z}$ \\
\hline
\end{tabular}


Appendix. Water-level measurements in selected wells, Diamond Valley, Nevada, 1960-90—Continued

\begin{tabular}{|c|c|c|c|c|c|c|c|}
\hline \multirow{2}{*}{\multicolumn{2}{|c|}{$\begin{array}{c}\text { U.S. Geological Survey } \\
\text { site designation }\end{array}$}} & \multirow{3}{*}{$\begin{array}{l}\text { Depth } \\
\text { of } \\
\text { well } \\
\text { (feet) }\end{array}$} & \multirow{3}{*}{$\begin{array}{c}\text { Land- } \\
\text { surface } \\
\text { altitude } \\
\text { (feet above } \\
\text { sea level) }\end{array}$} & \multicolumn{4}{|c|}{ Water-level measurement } \\
\hline & & & & \multirow[b]{2}{*}{ Date } & \multirow{2}{*}{$\begin{array}{l}\text { Feet } \\
\text { below } \\
\text { land } \\
\text { surface }\end{array}$} & \multirow[b]{2}{*}{ Status } & \multirow[b]{2}{*}{ Method } \\
\hline $\begin{array}{c}\text { Local } \\
\text { identification }\end{array}$ & $\begin{array}{c}\text { Standard } \\
\text { identiflcation }\end{array}$ & & & & & & \\
\hline & & & & $04-25-69$ & 67.4 & -- & $\mathbf{Z}$ \\
\hline & & & & $09-09-69$ & 78.5 & -- & $\mathrm{Z}$ \\
\hline & & & & $04-20-71$ & 64.6 & $\mathrm{~S}$ & $\mathbf{Z}$ \\
\hline & & & & $11-18-71$ & 85.8 & -- & $\mathbf{Z}$ \\
\hline & & & & $12-06-72$ & 64.8 & -- & $\mathrm{Z}$ \\
\hline & & & & $02-27-73$ & 77.6 & -- & $\mathrm{Z}$ \\
\hline & & & & $11-05-73$ & 85.3 & -- & $\mathbf{Z}$ \\
\hline & & & & $03-11-74$ & 77.1 & -- & $\mathrm{Z}$ \\
\hline & & & & $11-03-75$ & 86.1 & -- & $\mathrm{Z}$ \\
\hline & & & & $04-05-76$ & 84.3 & -- & $\mathbf{Z}$ \\
\hline & & & & $11-08-76$ & 94.2 & -- & $\mathbf{Z}$ \\
\hline & & & & $03-05-77$ & 84.0 & -- & $\mathbf{Z}$ \\
\hline & & & & $11-16-77$ & 92.8 & -- & $\mathbf{Z}$ \\
\hline & & & & $03-29-78$ & 86.4 & -- & $\bar{Z}$ \\
\hline & & & & $11-09-78$ & 94.4 & -- & $\mathrm{Z}$ \\
\hline & & & & $04-05-79$ & 88.1 & -- & $\mathrm{Z}$ \\
\hline & & & & $11-08-79$ & 102.2 & -- & Z \\
\hline & & & & $04-14-80$ & 115.1 & -. & $\mathbf{Z}$ \\
\hline & & & & $11-18-80$ & 103.8 & -- & $\mathrm{Z}$ \\
\hline & & & & $11-00-81$ & 119.0 & -- & $\mathrm{R}$ \\
\hline & & & & $04-06-82$ & 111.3 & -- & $\mathrm{Z}$ \\
\hline & & & & $11-10-82$ & 117.80 & -- & $\mathbf{Z}$ \\
\hline & & & & $03-30-83$ & 108.6 & -- & $\mathbf{Z}$ \\
\hline & & & & $11-09-83$ & 115.3 & -- & $\mathbf{Z}$ \\
\hline & & & & $11-08-84$ & 108.7 & -- & $\bar{Z}$ \\
\hline & & & & $12-04-85$ & 111.20 & -- & $\mathrm{Z}$ \\
\hline \multirow[t]{16}{*}{153 N21 E53 21DCAA1 } & 394004116002101 & 180 & 5,910 & $04-06-66$ & 57.59 & - & $\mathrm{S}$ \\
\hline & & & & $10-01-73$ & 75 & -- & $\mathrm{Z}$ \\
\hline & & & & $11-03-75$ & 77.4 & - & $Z$ \\
\hline & & & & $11-08-76$ & 84.3 & - & $\mathrm{Z}$ \\
\hline & & & & $11-14-77$ & 87.6 & $\therefore$ & $\mathrm{Z}$ \\
\hline & & & & $11-08-78$ & 89.8 & -- & $\mathrm{Z}$ \\
\hline & & & & $11-01-79$ & 97.4 & -- & $Z$ \\
\hline & & & & $11-01-80$ & 98.1 & -- & $\mathrm{Z}$ \\
\hline & & & & $11-01-81$ & 103.4 & -- & $\mathrm{Z}$ \\
\hline & & & & $11-10-82$ & 102.0 & -- & $\mathrm{Z}$ \\
\hline & & & & $11-09-83$ & 106.2 & -- & $\mathrm{Z}$ \\
\hline & & & & $11-08-84$ & 103.7 & -- & $\mathrm{Z}$ \\
\hline & & & & $12-04-85$ & 105.5 & -- & $\bar{Z}$ \\
\hline & & & & $11-13-86$ & 107.4 & -- & $\mathrm{Z}$ \\
\hline & & & & $11-18-87$ & 115.5 & -- & $\mathrm{Z}$ \\
\hline & & & & $10-21-88$ & 116.3 & -. & $\bar{Z}$ \\
\hline
\end{tabular}


Appendix. Water-level measurements in selected wells, Diamond Valley, Nevada, 1960-90-Continued

\begin{tabular}{|c|c|c|c|c|c|c|c|c|}
\hline \multirow{2}{*}{\multicolumn{3}{|c|}{$\begin{array}{l}\text { U.S. Geological Survey } \\
\text { site designation }\end{array}$}} & \multirow{3}{*}{$\begin{array}{c}\text { Depth } \\
\text { of } \\
\text { well } \\
\text { (feet) }\end{array}$} & \multirow{3}{*}{$\begin{array}{c}\text { Land- } \\
\text { surface } \\
\text { altitude } \\
\text { (feet above } \\
\text { sea level) }\end{array}$} & \multicolumn{4}{|c|}{ Water-level measurement } \\
\hline & & & & & \multirow[b]{2}{*}{ Date } & \multirow{2}{*}{$\begin{array}{c}\text { Feet } \\
\text { below } \\
\text { land } \\
\text { surface }\end{array}$} & \multirow[b]{2}{*}{ Status } & \multirow[b]{2}{*}{ Method } \\
\hline & $\begin{array}{l}\text { Local } \\
\text { identification }\end{array}$ & $\begin{array}{l}\text { Standard } \\
\text { identification }\end{array}$ & & & & & & \\
\hline \multirow{38}{*}{\multicolumn{2}{|c|}{153 N21 E53 22BDBB1 }} & 394032115594401 & 180 & 5,904 & $05-12-63$ & 51 & -- & -- \\
\hline & & & & & $11-00-63$ & 51.9 & -- & $\mathbf{R}$ \\
\hline & & & & & $04-00-64$ & 53.4 & -- & $\mathrm{R}$ \\
\hline & & & & & $11-00-64$ & 54.6 & -- & $\mathrm{R}$ \\
\hline & & & & & $04-00-65$ & 51.4 & -- & $\mathrm{R}$ \\
\hline & & & & & $11-00-65$ & 54.6 & -- & $\mathrm{R}$ \\
\hline & & & & & $04-06-66$ & 53.06 & -- & $\mathrm{Z}$ \\
\hline & & & & & $11-00-66$ & 57.2 & -- & $\mathrm{R}$ \\
\hline & & & & & $04-00-67$ & 54.2 & -- & $\mathrm{R}$ \\
\hline & & & & & $11-00-67$ & 65.5 & -- & $\mathrm{R}$ \\
\hline & & & & & $04-00-68$ & 55.9 & -. & $\mathbf{R}$ \\
\hline & & & & & $08-23-68$ & 64.9 & $\mathrm{~S}$ & $\mathrm{Z}$ \\
\hline & & & & & $04-24-69$ & 56.9 & -- & $\mathrm{Z}$ \\
\hline & & & & & $09-09-69$ & 64.0 & $S$ & $\mathrm{Z}$ \\
\hline & & & & & $04-20-71$ & 63.5 & -- & $\mathrm{Z}$ \\
\hline & & & & & $11-18-71$ & 61.5 & -. & $\mathrm{Z}$ \\
\hline & & & & & $02-24-72$ & 61.3 & -- & $\mathrm{Z}$ \\
\hline & & & & & $02-27-73$ & 59.9 & -- & $\mathrm{Z}$ \\
\hline & & & & & $07-23-73$ & 81.4 & $\mathrm{~S}$ & $\mathrm{Z}$ \\
\hline & & & & & $10-30-73$ & 73.2 & -- & $\mathrm{Z}$ \\
\hline & & & & & $03-11-74$ & 63.5 & -- & $\mathrm{Z}$ \\
\hline & & & & & $11-03-75$ & 71.3 & -. & $\mathrm{Z}$ \\
\hline & & & & & $04-05-76$ & 71.5 & -- & $\mathrm{Z}$ \\
\hline & & & & & $11-08-76$ & 79.0 & -- & $\mathrm{Z}$ \\
\hline & & & & & $03-05-77$ & 72.5 & -- & $\mathrm{Z}$ \\
\hline & & & & & $03-29-78$ & 82.7 & .. & $\mathrm{Z}$ \\
\hline & & & & & $11-09-78$ & 82.1 & -- & $\mathrm{Z}$ \\
\hline & & & & & $04-09-79$ & 76.6 & - & $\mathrm{Z}$ \\
\hline & & & & & $11-08-79$ & 87.1 & -- & $\mathrm{Z}$ \\
\hline & & & & & $04-14-80$ & 83.1 & - & $\mathrm{z}$ \\
\hline & & & & & $11-20-80$ & 91.3 & -- & $\mathrm{Z}$ \\
\hline & & & & & $11-00-81$ & 99.3 & -- & $\mathbf{R}$ \\
\hline & & & & & $04-06-82$ & 97.9 & -- & $\mathrm{Z}$ \\
\hline & & & & & $11-10-82$ & 98.70 & -- & $\mathrm{Z}$ \\
\hline & & & & & $03-30-83$ & 98.8 & -. & $\mathrm{Z}$ \\
\hline & & & & & $11-09-83$ & 101.30 & -- & $\mathrm{Z}$ \\
\hline & & & & & $11-08-84$ & 100.60 & -- & $\mathrm{Z}$ \\
\hline & & & & & $12-04-85$ & 102.80 & -- & $\bar{Z}$ \\
\hline \multirow[t]{5}{*}{153} & N21 E53 23ACCC1 & 394036115580101 & 172 & 5,903 & $09-18-60$ & 44.0 & -. & -- \\
\hline & & & & & $11-00-63$ & 51.6 & -- & $\mathrm{R}$ \\
\hline & & & & & $04-00-64$ & 51.0 & -- & $\mathrm{R}$ \\
\hline & & & & & $04-00-65$ & 45.6 & -- & $\mathrm{R}$ \\
\hline & & & & & $04-06-66$ & 47.30 & -- & $\mathrm{Z}$ \\
\hline
\end{tabular}


Appendix. Water-level measurements in selected wells, Diamond Valley, Nevada, 1960-90-Continued

\begin{tabular}{|c|c|c|c|c|c|c|c|c|}
\hline \multirow{2}{*}{\multicolumn{3}{|c|}{$\begin{array}{l}\text { U.S. Geological Survey } \\
\text { site designation }\end{array}$}} & \multirow{3}{*}{$\begin{array}{c}\text { Depth } \\
\text { of } \\
\text { well } \\
\text { (feet) }\end{array}$} & \multirow{3}{*}{$\begin{array}{c}\text { Land- } \\
\text { surface } \\
\text { altitude } \\
\text { (feet above } \\
\text { sea level) }\end{array}$} & \multicolumn{4}{|c|}{ Water-level measurement } \\
\hline & & & & & \multirow[b]{2}{*}{ Date } & \multirow{2}{*}{$\begin{array}{c}\text { Feet } \\
\text { below } \\
\text { land } \\
\text { surface }\end{array}$} & \multirow[b]{2}{*}{ Status } & \multirow[b]{2}{*}{ Method } \\
\hline & $\begin{array}{l}\text { Local } \\
\text { Identification }\end{array}$ & $\begin{array}{l}\text { Standard } \\
\text { Identification }\end{array}$ & & & & & & \\
\hline \multirow{26}{*}{\multicolumn{2}{|c|}{153 N21 E53 23ACCC1 }} & (Continued) & & & $11-00-66$ & 56.7 & -- & $\mathrm{R}$ \\
\hline & & & & & $04-00-67$ & 48.3 & -- & $\mathrm{R}$ \\
\hline & & & & & $11-00-67$ & 60.7 & -- & $\mathbf{R}$ \\
\hline & & & & & $04-00-68$ & 48.9 & -- & $\mathrm{R}$ \\
\hline & & & & & $08-26-68$ & 55.3 & -- & $z$ \\
\hline & & & & & $04-23-69$ & 50.6 & -- & $\mathrm{z}$ \\
\hline & & & & & $09-09-69$ & 60.1 & $S$ & $\mathrm{Z}$ \\
\hline & & & & & $11-18-71$ & 55.0 & -- & $\mathrm{Z}$ \\
\hline & & & & & $02-24-72$ & 51.0 & -- & $\mathrm{Z}$ \\
\hline & & & & & $12-06-72$ & 47.4 & -. & $\mathrm{Z}$ \\
\hline & & & & & $02-27-73$ & 53.2 & -- & $\mathrm{Z}$ \\
\hline & & & & & $07-24-73$ & 76.7 & $S$ & $\mathrm{Z}$ \\
\hline & & & & & $11-02-73$ & 54.6 & -- & $\mathrm{Z}$ \\
\hline & & & & & $03-12-74$ & 56.2 & -- & $\mathrm{Z}$ \\
\hline & & & & & $11-05-75$ & 62.8 & -- & $\mathrm{z}$ \\
\hline & & & & & $04-06-76$ & 64.3 & -- & $\mathrm{Z}$ \\
\hline & & & & & $11-15-77$ & 79.5 & -- & $\mathrm{Z}$ \\
\hline & & & & & $03-28-78$ & 74.4 & -- & $\mathrm{Z}$ \\
\hline & & & & & $11-08-78$ & 78.1 & -- & $\mathrm{z}$ \\
\hline & & & & & 04-05-79 & 73.3 & - & $\mathrm{Z}$ \\
\hline & & & & & $11-08-79$ & 85.5 & -- & $\mathrm{Z}$ \\
\hline & & & & & $04-14-80$ & 77.6 & -- & z \\
\hline & & & & & $11-20-80$ & 86.6 & -- & $\mathrm{Z}$ \\
\hline & & & & & $11-00-81$ & 98.7 & -- & $\mathrm{Z}$ \\
\hline & & & & & $04-05-82$ & 95.3 & -- & $\mathrm{Z}$ \\
\hline & & & & & $03-30-83$ & 101.0 & -- & $\mathrm{Z}$ \\
\hline \multirow[t]{17}{*}{153} & N21 E53 23BDBB1 & 394021115584801 & 216 & 5,903 & $04-06-66$ & 50.29 & -- & $S$ \\
\hline & & & & & $10-01-73$ & 70.1 & -- & $\mathrm{Z}$ \\
\hline & & & & & $11-03-75$ & 70.3 & -- & $\mathrm{Z}$ \\
\hline & & & & & $03-01-76$ & 64.8 & -- & $\mathrm{Z}$ \\
\hline & & & & & $11-08-76$ & 76.9 & -- & $\mathrm{z}$ \\
\hline & & & & & $11-14-77$ & 76.3 & -- & $\mathrm{Z}$ \\
\hline & & & & & $11-08-78$ & 80.4 & -- & Z \\
\hline & & & & & $11-01-79$ & 87.4 & -- & $\mathrm{Z}$ \\
\hline & & & & & $11-01-80$ & 89.1 & -- & Z \\
\hline & & & & & $11-01-81$ & 98.4 & -- & $\mathrm{Z}$ \\
\hline & & & & & $11-09-82$ & 96.6 & -- & $\mathrm{z}$ \\
\hline & & & & & $11-09-83$ & 101.7 & -- & Z \\
\hline & & & & & $11-09-84$ & 97.2 & -- & $\mathrm{Z}$ \\
\hline & & & & & $11-13-86$ & 99.1 & -- & Z \\
\hline & & & & & $11-18-87$ & 100.6 & $-\cdot$ & $\mathrm{Z}$ \\
\hline & & & & & $10-19-88$ & 103.9 & -- & Z \\
\hline & & & & & $10-24-89$ & 106.3 & -- & Z \\
\hline
\end{tabular}


Appendix. Water-level measurements in selected wells, Diamond Valley, Nevada, 1960-90—Continued

\begin{tabular}{|c|c|c|c|c|c|c|c|c|}
\hline \multirow{2}{*}{\multicolumn{3}{|c|}{$\begin{array}{l}\text { U.S. Geological Survey } \\
\text { site designation }\end{array}$}} & \multirow{3}{*}{$\begin{array}{c}\text { Depth } \\
\text { of } \\
\text { well } \\
\text { (feet) }\end{array}$} & \multirow{3}{*}{$\begin{array}{c}\text { Land- } \\
\text { surface } \\
\text { altitude } \\
\text { (feet above } \\
\text { sea level) }\end{array}$} & \multicolumn{4}{|c|}{ Water-level measurement } \\
\hline & & & & & \multirow[b]{2}{*}{ Date } & \multirow{2}{*}{$\begin{array}{l}\text { Feet } \\
\text { below } \\
\text { land } \\
\text { surface }\end{array}$} & \multirow[b]{2}{*}{ Status } & \multirow[b]{2}{*}{ Method } \\
\hline & $\begin{array}{l}\text { Local } \\
\text { identification }\end{array}$ & $\begin{array}{c}\text { Standard } \\
\text { identification }\end{array}$ & & & & & & \\
\hline \multirow{27}{*}{\multicolumn{2}{|c|}{153 N21 E53 24CDDD1 }} & 393956115571101 & 280 & 5,918 & $06-20-64$ & 62.0 & -- & -- \\
\hline & & & & & $04-06-66$ & 62.40 & -- & $\mathrm{Z}$ \\
\hline & & & & & $11-00-67$ & 74.6 & .- & $\mathbf{R}$ \\
\hline & & & & & $04-00-68$ & 65.6 & -- & $\mathrm{R}$ \\
\hline & & & & & $08-28-68$ & 77.1 & -- & $\mathrm{Z}$ \\
\hline & & & & & $02-18-69$ & 67.3 & -- & $\mathrm{Z}$ \\
\hline & & & & & $09-18-69$ & 73.7 & $\mathrm{~S}$ & $\mathrm{Z}$ \\
\hline & & & & & $04-20-71$ & 68.2 & -- & $\mathrm{Z}$ \\
\hline & & & & & $11-18-71$ & 72.1 & -- & $\mathrm{Z}$ \\
\hline & & & & & $12-06-72$ & 72.4 & - & $\mathrm{z}$ \\
\hline & & & & & $04-00-73$ & 73.3 & -- & $\mathrm{Z}$ \\
\hline & & & & & $07-24-73$ & 92.5 & $\mathrm{~S}$ & $\mathrm{Z}$ \\
\hline & & & & & $03-12-74$ & 73.1 & -- & $\mathrm{Z}$ \\
\hline & & & & & $11-05-75$ & 78.9 & -- & $\mathrm{Z}$ \\
\hline & & & & & $04-06-76$ & 75.2 & -- & $\mathrm{Z}$ \\
\hline & & & & & $11-08-76$ & 86.5 & -- & $\mathrm{Z}$ \\
\hline & & & & & $03-05-77$ & 78.4 & -- & $\mathrm{Z}$ \\
\hline & & & & & $11-14-77$ & 89.2 & -- & $\mathrm{Z}$ \\
\hline & & & & & $03-29-78$ & 82.9 & -- & $\mathrm{z}$ \\
\hline & & & & & $11-08-78$ & 90.4 & -- & $\mathrm{Z}$ \\
\hline & & & & & $04-05-79$ & 84.6 & -- & $\mathrm{z}$ \\
\hline & & & & & $11-08-79$ & 104.7 & -- & $\mathrm{Z}$ \\
\hline & & & & & $04-14-80$ & 94.2 & -- & $\mathrm{Z}$ \\
\hline & & & & & $11-20-80$ & 98.6 & -. & $\mathrm{z}$ \\
\hline & & & & & $11-00-81$ & 101.3 & -- & $\mathbf{R}$ \\
\hline & & & & & $04-00-82$ & 98.5 & -- & $\mathrm{R}$ \\
\hline & & & & & $03-30-83$ & 96.9 & -- & $\mathrm{Z}$ \\
\hline \multirow[t]{15}{*}{153} & N21 E53 26AACCl & 393942115580401 & 181 & 5,907 & $09-13-61$ & 48 & -- & -- \\
\hline & & & & & $04-00-64$ & 53.4 & -- & $\mathrm{R}$ \\
\hline & & & & & $11-00-64$ & 56.0 & -- & $\mathbf{R}$ \\
\hline & & & & & $04-00-65$ & 51.2 & -- & $\mathbf{R}$ \\
\hline & & & & & $04-06-66$ & 58.40 & -- & $\mathrm{Z}$ \\
\hline & & & & & $11-00-66$ & 60.0 & -- & $\mathbf{R}$ \\
\hline & & & & & $04-00-67$ & 54.9 & .. & $\mathbf{R}$ \\
\hline & & & & & $11-00-67$ & 68.7 & -- & $\mathbf{R}$ \\
\hline & & & & & $04-00-68$ & 55.7 & -. & $\mathbf{R}$ \\
\hline & & & & & $02-18-69$ & 59.0 & - & $\mathrm{Z}$ \\
\hline & & & & & $09-09-69$ & 68.0 & $\mathrm{~S}$ & $\mathrm{Z}$ \\
\hline & & & & & $04-15-71$ & 61.8 & -- & $\mathrm{Z}$ \\
\hline & & & & & $02-23-72$ & 59.9 & -- & $\mathrm{Z}$ \\
\hline & & & & & $12-06-72$ & 62.4 & -- & $\mathrm{Z}$ \\
\hline & & & & & $02-27-73$ & 63.0 & -- & $\mathrm{Z}$ \\
\hline
\end{tabular}


Appendix. Water-level measurements in selected wells, Diamond Valley, Nevada, 1960-90-Continued

\begin{tabular}{|c|c|c|c|c|c|c|c|c|}
\hline \multirow{2}{*}{\multicolumn{3}{|c|}{$\begin{array}{l}\text { U.S. Geological Survey } \\
\text { site designation }\end{array}$}} & \multirow{3}{*}{$\begin{array}{l}\text { Depth } \\
\text { of } \\
\text { well } \\
\text { (feet) }\end{array}$} & \multirow{3}{*}{$\begin{array}{c}\text { Land- } \\
\text { surface } \\
\text { altitude } \\
\text { (feet above } \\
\text { sea level) }\end{array}$} & \multicolumn{4}{|c|}{ Water-level measurement } \\
\hline & & & & & \multirow[b]{2}{*}{ Date } & \multirow{2}{*}{$\begin{array}{l}\text { Feet } \\
\text { below } \\
\text { land } \\
\text { surface }\end{array}$} & \multirow[b]{2}{*}{ Status } & \multirow[b]{2}{*}{ Method } \\
\hline & $\begin{array}{l}\text { Local } \\
\text { identification }\end{array}$ & $\begin{array}{l}\text { Standard } \\
\text { identiflcation }\end{array}$ & & & & & & \\
\hline \multirow[t]{8}{*}{153} & N21 E53 26AACC1 & Continued) & & & $11-03-73$ & 71.4 & -- & $\mathrm{Z}$ \\
\hline & & & & & $03-12-74$ & 63.6 & -- & $\mathrm{Z}$ \\
\hline & & & & & $11-05-75$ & 72.8 & -- & $\mathrm{Z}$ \\
\hline & & & & & $04-06-76$ & 70.1 & -- & $\mathrm{Z}$ \\
\hline & & & & & $11-15-77$ & 74.7 & -- & $\mathrm{Z}$ \\
\hline & & & & & $03-29-78$ & 71.6 & -- & $\mathrm{Z}$ \\
\hline & & & & & $11-00-81$ & 103.7 & -- & $\mathrm{R}$ \\
\hline & & & & & $03-30-83$ & 94.0 & -- & $\mathrm{Z}$ \\
\hline \multirow[t]{17}{*}{153} & N21 E53 27ACAA1 & 393940115591701 & -- & 5,909 & $04-06-66$ & 55.18 & -- & $\mathrm{s}$ \\
\hline & & & & & $10-01-73$ & 77.2 & -- & $\mathrm{Z}$ \\
\hline & & & & & $11-03-75$ & 78.0 & -- & $\mathrm{Z}$ \\
\hline & & & & & $03-01-76$ & 73.0 & -- & $\mathrm{Z}$ \\
\hline & & & & & $11-08-76$ & 87.1 & -- & $\mathrm{Z}$ \\
\hline & & & & & $11-14-77$ & -- & $\mathbf{P}$ & -- \\
\hline & & & & & $11-08-78$ & 86.1 & -- & $\mathrm{Z}$ \\
\hline & & & & & $11-01-79$ & 96.8 & -- & $\mathrm{Z}$ \\
\hline & & & & & $11-01-80$ & 96.7 & -- & $\mathrm{Z}$ \\
\hline & & & & & $11-01-81$ & 105.5 & -- & $\mathrm{z}$ \\
\hline & & & & & $11-10-82$ & -- & $P$ & -. \\
\hline & & & & & $11-09-83$ & 112.3 & -- & Z \\
\hline & & & & & $11-08-84$ & 105.2 & -- & Z \\
\hline & & & & & $11-13-86$ & 114.1 & -- & $\mathrm{Z}$ \\
\hline & & & & & $11-19-87$ & 115.3 & .- & $\mathrm{Z}$ \\
\hline & & & & & $10-21-88$ & 117.9 & -- & $\mathrm{Z}$ \\
\hline & & & & & $10-24-89$ & 117.9 & -- & $\mathrm{Z}$ \\
\hline \multirow[t]{11}{*}{153} & N21 E53 28CCAA1 & 393915116011001 & 186 & 5,942 & $04-06-66$ & 85.60 & -- & $\mathrm{s}$ \\
\hline & & & & & $10-01-73$ & 104.3 & -- & $\mathrm{Z}$ \\
\hline & & & & & $11-03-75$ & 109.4 & -- & $\mathrm{Z}$ \\
\hline & & & & & $03-01-76$ & -- & $\mathrm{P}$ & -- \\
\hline & & & & & $11-08-76$ & 116.7 & -- & $\mathrm{Z}$ \\
\hline & & & & & $11-14-77$ & 120.1 & -- & $\mathrm{Z}$ \\
\hline & & & & & $11-08-78$ & 115.1 & -- & $\mathrm{Z}$ \\
\hline & & & & & $11-01-79$ & 127.2 & -- & Z \\
\hline & & & & & $11-01-80$ & 129.4 & -- & Z \\
\hline & & & & & $11-01-81$ & 138.8 & -- & $\mathrm{Z}$ \\
\hline & & & & & $11-09-83$ & 127.9 & -- & $\mathrm{Z}$ \\
\hline \multirow[t]{10}{*}{153} & N21 E53 33AACC1 & 393838116002401 & 112 & 5,920 & $03-00-64$ & 60.0 & -- & $\mathrm{Z}$ \\
\hline & & & & & $11-00-64$ & 56.0 & -- & $\mathrm{Z}$ \\
\hline & & & & & $03-00-65$ & 59.6 & -- & $\mathrm{Z}$ \\
\hline & & & & & $11-00-65$ & 62.0 & -- & $\mathrm{Z}$ \\
\hline & & & & & $03-00-66$ & 61.7 & -- & $\mathrm{Z}$ \\
\hline & & & & & $04-06-66$ & 61.67 & -. & $\mathrm{s}$ \\
\hline & & & & & $03-00-67$ & 62.5 & -- & $\mathrm{Z}$ \\
\hline & & & & & $11-00-67$ & 71.9 & -- & $\mathrm{Z}$ \\
\hline & & & & & $03-00-68$ & 64.3 & -- & $\mathrm{Z}$ \\
\hline & & & & & $11-00-68$ & 73.0 & -. & $\mathrm{Z}$ \\
\hline
\end{tabular}


Appendix. Water-level measurements in selected wells, Diamond Valley, Nevada, 1960-90-Continued

\begin{tabular}{|c|c|c|c|c|c|c|c|c|}
\hline \multirow{2}{*}{\multicolumn{3}{|c|}{$\begin{array}{l}\text { U.S. Geological Survey } \\
\text { site designation }\end{array}$}} & \multirow{3}{*}{$\begin{array}{c}\text { Depth } \\
\text { of } \\
\text { well } \\
\text { (feet) }\end{array}$} & \multirow{3}{*}{$\begin{array}{c}\text { Land- } \\
\text { surface } \\
\text { altitude } \\
\text { (feet above } \\
\text { sea level) }\end{array}$} & \multicolumn{4}{|c|}{ Water-level measurement } \\
\hline & & & & & \multirow[b]{2}{*}{ Date } & \multirow{2}{*}{$\begin{array}{c}\text { Feet } \\
\text { below } \\
\text { land } \\
\text { surface }\end{array}$} & \multirow[b]{2}{*}{ Status } & \multirow[b]{2}{*}{ Method } \\
\hline & $\begin{array}{l}\text { Local } \\
\text { Identiflcation }\end{array}$ & $\begin{array}{l}\text { Standard } \\
\text { Identification }\end{array}$ & & & & & & \\
\hline \multirow{18}{*}{\multicolumn{3}{|c|}{153 N21 E53 33AACC1 (Continued) }} & & & $03-00-69$ & 65.1 & -- & $\mathrm{Z}$ \\
\hline & & & & & $11-00-69$ & 69.6 & -- & $\mathrm{Z}$ \\
\hline & & & & & $11-00-71$ & 69.0 & -- & $\mathrm{Z}$ \\
\hline & & & & & $03-00-72$ & 69.3 & -- & $\mathrm{Z}$ \\
\hline & & & & & $11-00-73$ & 77.3 & -- & $\mathrm{Z}$ \\
\hline & & & & & $03-00-74$ & 74.4 & -- & $\mathrm{Z}$ \\
\hline & & & & & $11-00-75$ & 83.2 & -- & $\mathrm{Z}$ \\
\hline & & & & & $03-00-76$ & 77.7 & -- & $\mathrm{Z}$ \\
\hline & & & & & $11-00-76$ & 91.6 & -- & $\mathrm{Z}$ \\
\hline & & & & & $03-00-77$ & 82.6 & -- & $\mathrm{Z}$ \\
\hline & & & & & $11-00-77$ & 96.1 & -- & $\mathrm{Z}$ \\
\hline & & & & & $03-00-78$ & 91.9 & -- & $\mathrm{Z}$ \\
\hline & & & & & $11-00-78$ & 89.3 & -- & $\mathrm{Z}$ \\
\hline & & & & & $03-00-79$ & 85.4 & -- & $\mathrm{Z}$ \\
\hline & & & & & $11-00-79$ & 94.9 & -- & $\bar{Z}$ \\
\hline & & & & & $11-00-80$ & 95.9 & -- & $\mathrm{Z}$ \\
\hline & & & & & $11-00-81$ & 102.5 & -- & $\mathrm{Z}$ \\
\hline & & & & & $03-00-82$ & 99.2 & -- & $\mathrm{Z}$ \\
\hline \multirow{20}{*}{\multicolumn{2}{|c|}{153 N21 E53 34BCAA1 }} & 393849116000201 & 128 & 5,920 & $07-10-61$ & 60 & - & -. \\
\hline & & & & & $04-00-64$ & 47.5 & -- & $\mathrm{R}$ \\
\hline & & & & & $11-00-64$ & 61.4 & -- & $\mathrm{R}$ \\
\hline & & & & & $04-05-66$ & 62.34 & -- & $\mathrm{Z}$ \\
\hline & & & & & $11-00-66$ & 64.7 & -. & $\mathrm{R}$ \\
\hline & & & & & $04-00-67$ & 62.8 & -- & $\mathrm{R}$ \\
\hline & & & & & $11-00-67$ & 73.1 & -- & $\mathrm{R}$ \\
\hline & & & & & $04-00-68$ & 64.4 & -- & $\mathrm{R}$ \\
\hline & & & & & $08-22-68$ & 73.5 & $S$ & $\mathrm{Z}$ \\
\hline & & & & & $02-18-69$ & 64.8 & -- & $\mathrm{Z}$ \\
\hline & & & & & $09-09-69$ & 69.6 & $S$ & $\mathrm{Z}$ \\
\hline & & & & & $04-15-71$ & 69.4 & -- & $\mathrm{Z}$ \\
\hline & & & & & $02-23-72$ & 67.7 & -- & $\mathrm{Z}$ \\
\hline & & & & & $12-05-72$ & 70.0 & -- & $\mathrm{Z}$ \\
\hline & & & & & $02-27-73$ & 68.5 & -- & $\mathrm{Z}$ \\
\hline & & & & & $04-00-73$ & 65.4 & -- & $\mathrm{R}$ \\
\hline & & & & & $07-24-73$ & -- & $\mathrm{P}$ & -- \\
\hline & & & & & $11-03-73$ & 75.9 & -. & $\mathrm{Z}$ \\
\hline & & & & & $03-11-74$ & 73.1 & -- & $\mathrm{Z}$ \\
\hline & & & & & $04-14-80$ & 87.5 & -- & $\mathrm{Z}$ \\
\hline \multirow[t]{5}{*}{153} & N21 E53 34DDBB1 & 393816115591701 & 157 & 5,919 & $04-05-66$ & 57.82 & -- & $S$ \\
\hline & & & & & $10-01-73$ & 71.5 & -- & $\mathrm{Z}$ \\
\hline & & & & & $11-03-75$ & 77.6 & -- & $\mathrm{Z}$ \\
\hline & & & & & $03-01-76$ & 72.3 & -- & $\mathrm{Z}$ \\
\hline & & & & & $11-08-76$ & 80.0 & -- & $\mathrm{Z}$ \\
\hline
\end{tabular}


Appendix. Water-level measurements in selected wells, Diamond Valley, Nevada, 1960-90-Continued

\begin{tabular}{|c|c|c|c|c|c|c|c|c|}
\hline \multirow{2}{*}{\multicolumn{3}{|c|}{$\begin{array}{c}\text { U.S. Geological Survey } \\
\text { site desIgnation }\end{array}$}} & \multirow{3}{*}{$\begin{array}{l}\text { Depth } \\
\text { of } \\
\text { well } \\
\text { (feet) }\end{array}$} & \multirow{3}{*}{$\begin{array}{l}\text { Land- } \\
\text { surface } \\
\text { altitude } \\
\text { (feet above } \\
\text { sea level) }\end{array}$} & \multicolumn{4}{|c|}{ Water-level measurement } \\
\hline & & & & & \multirow[b]{2}{*}{ Date } & \multirow{2}{*}{$\begin{array}{c}\text { Feet } \\
\text { below } \\
\text { land } \\
\text { surface }\end{array}$} & \multirow[b]{2}{*}{ Status } & \multirow[b]{2}{*}{ Method } \\
\hline & $\begin{array}{l}\text { Local } \\
\text { identification }\end{array}$ & $\begin{array}{c}\text { Standard } \\
\text { identification }\end{array}$ & & & & & & \\
\hline \multirow[t]{12}{*}{153} & N21 E53 34DDBB1 ( & ontinued) & & & $11-14-77$ & 84.4 & -- & $\mathrm{Z}$ \\
\hline & & & & & $11-01-79$ & 94.9 & -- & $\mathrm{Z}$ \\
\hline & & & & & $11-01-80$ & 97.1 & -- & $\mathrm{z}$ \\
\hline & & & & & $11-01-81$ & 108.0 & -- & $\mathrm{Z}$ \\
\hline & & & & & $11-12-82$ & 105.8 & -- & $\mathrm{Z}$ \\
\hline & & & & & $11-09-83$ & 110.3 & -- & $\mathrm{z}$ \\
\hline & & & & & $11-08-84$ & 108.6 & -- & $\mathrm{Z}$ \\
\hline & & & & & $11-05-86$ & 109.0 & -- & $\mathrm{Z}$ \\
\hline & & & & & $11-18-87$ & 109.4 & -- & $\mathrm{Z}$ \\
\hline & & & & & $10-20-88$ & 113.1 & -- & $\mathrm{Z}$ \\
\hline & & & & & $10-24-89$ & 109.6 & -- & $\mathrm{Z}$ \\
\hline & & & & & $09-20-90$ & -- & $\mathbf{P}$ & -- \\
\hline \multirow[t]{20}{*}{153} & N21 E53 35CCDD1 & 393809115583901 & 195 & 5,927 & $06-13-61$ & 54.0 & -- & -- \\
\hline & & & & & $02-23-72$ & 74.9 & -- & $\mathrm{Z}$ \\
\hline & & & & & $12-05-72$ & 69.3 & -- & $\mathrm{Z}$ \\
\hline & & & & & $02-27-73$ & 70.2 & -- & $\mathrm{Z}$ \\
\hline & & & & & $11-03-73$ & 82.1 & -- & $\mathrm{Z}$ \\
\hline & & & & & $03-12-74$ & 73.6 & -- & $\mathrm{Z}$ \\
\hline & & & & & $11-04-75$ & 79.7 & -- & $\mathrm{Z}$ \\
\hline & & & & & $04-06-76$ & 75.7 & -- & $\mathrm{Z}$ \\
\hline & & & & & $11-08-76$ & 82.7 & -- & $\mathrm{Z}$ \\
\hline & & & & & $03-05-77$ & 81.9 & -- & $\mathrm{z}$ \\
\hline & & & & & $11-15-77$ & 87.3 & - & $\mathrm{Z}$ \\
\hline & & & & & $03-29-78$ & 83.4 & -- & $\mathrm{Z}$ \\
\hline & & & & & $11-09-78$ & 91.4 & -- & $\mathrm{Z}$ \\
\hline & & & & & $04-06-79$ & 90.8 & -- & $\mathrm{Z}$ \\
\hline & & & & & $11-08-79$ & 109.4 & -- & $\bar{Z}$ \\
\hline & & & & & $04-14-80$ & 101.6 & -- & $\mathrm{Z}$ \\
\hline & & & & & $11-19-80$ & 113.2 & -- & $\mathrm{Z}$ \\
\hline & & & & & $11-00-81$ & 122.4 & -- & $\mathbf{R}$ \\
\hline & & & & & $04-06-82$ & 118.7 & -- & $\mathrm{Z}$ \\
\hline & & & & & $03-30-83$ & 121.6 & -- & $\mathrm{Z}$ \\
\hline \multirow[t]{10}{*}{153} & N21 E53 36ACAB1 & 393850115570101 & 152 & 5,935 & $08-29-60$ & 61.5 & -- & -- \\
\hline & & & & & $11-00-63$ & 70.5 & -- & $\mathbf{R}$ \\
\hline & & & & & $04-00-64$ & 55.9 & -- & $\mathbf{R}$ \\
\hline & & & & & $11-00-64$ & 50.1 & -- & $\mathbf{R}$ \\
\hline & & & & & $04-05-66$ & 69.10 & -- & $\mathrm{Z}$ \\
\hline & & & & & $11-00-67$ & 97.2 & -- & $\mathbf{R}$ \\
\hline & & & & & $04-00-68$ & 81.8 & -- & $\mathbf{R}$ \\
\hline & & & & & $02-18-69$ & 72.1 & -- & $\mathrm{z}$ \\
\hline & & & & & $09-09-69$ & 83.1 & $S$ & $\mathrm{Z}$ \\
\hline & & & & & $04-15-71$ & 76.6 & -- & Z \\
\hline
\end{tabular}


Appendix. Water-level measurements in selected wells, Diamond Valley, Nevada, 1960-90-Continued

\begin{tabular}{|c|c|c|c|c|c|c|c|c|}
\hline \multirow{2}{*}{\multicolumn{3}{|c|}{$\begin{array}{l}\text { U.S. Geological Survey } \\
\text { site desIgnation }\end{array}$}} & \multirow{3}{*}{$\begin{array}{c}\text { Depth } \\
\text { of } \\
\text { well } \\
\text { (feet) }\end{array}$} & \multirow{3}{*}{$\begin{array}{c}\text { Land- } \\
\text { surface } \\
\text { altitude } \\
\text { (feet above } \\
\text { sea level) }\end{array}$} & \multicolumn{4}{|c|}{ Water-level measurement } \\
\hline & & & & & \multirow[b]{2}{*}{ Date } & \multirow{2}{*}{$\begin{array}{c}\text { Feet } \\
\text { below } \\
\text { land } \\
\text { surface }\end{array}$} & \multirow[b]{2}{*}{ Status } & \multirow[b]{2}{*}{ Method } \\
\hline & $\begin{array}{c}\text { Local } \\
\text { identification }\end{array}$ & $\begin{array}{c}\text { Standard } \\
\text { identiflcation }\end{array}$ & & & & & & \\
\hline \multirow{8}{*}{\multicolumn{3}{|c|}{153 N21 E53 36ACAB1 (Continued) }} & & & $11-17-71$ & 81.7 & -- & $\mathrm{Z}$ \\
\hline & & & & & $12-05-72$ & 79.2 & -- & $\mathrm{Z}$ \\
\hline & & & & & $02-23-73$ & 75.7 & - & $\mathrm{Z}$ \\
\hline & & & & & $07-24-73$ & -- & $\mathbf{P}$ & -- \\
\hline & & & & & $11-03-73$ & 99.7 & -- & $\mathrm{Z}$ \\
\hline & & & & & $03-12-74$ & 89.7 & -- & $\mathrm{Z}$ \\
\hline & & & & & $04-14-80$ & 108.2 & -- & Z \\
\hline & & & & & $03-30-83$ & 99.6 & -- & $\mathrm{T}$ \\
\hline \multirow{30}{*}{\multicolumn{2}{|c|}{153 N21 E53 36CDDD1 }} & 393810115571501 & -- & 5,941 & $11-00-63$ & 89.8 & -- & $\mathrm{R}$ \\
\hline & & & & & $04-00-64$ & 80.5 & -- & $\mathbf{R}$ \\
\hline & & & & & $11-00-64$ & 85.1 & -- & $\mathbf{R}$ \\
\hline & & & & & $04-05-66$ & 78.77 & -- & $\mathrm{Z}$ \\
\hline & & & & & $11-00-66$ & 91.0 & -- & $\mathbf{R}$ \\
\hline & & & & & $04-00-67$ & 83.0 & -- & $\mathbf{R}$ \\
\hline & & & & & $11-00-67$ & 96.3 & -- & $\mathbf{R}$ \\
\hline & & & & & $04-00-68$ & 89.8 & -- & $\mathbf{R}$ \\
\hline & & & & & $08-21-68$ & -- & $\mathrm{P}$ & -- \\
\hline & & & & & $02-18-69$ & 83.9 & -.- & $\mathrm{Z}$ \\
\hline & & & & & $09-09-69$ & 94.9 & $S$ & $\mathrm{Z}$ \\
\hline & & & & & $11-17-71$ & 82.1 & -- & $\mathrm{Z}$ \\
\hline & & & & & $02-23-72$ & 83.0 & -- & $\mathrm{Z}$ \\
\hline & & & & & $02-27-73$ & 89.8 & -- & $\mathrm{Z}$ \\
\hline & & & & & $07-24-73$ & -- & $\mathbf{P}$ & -- \\
\hline & & & & & $11-03-73$ & 107.80 & -- & $\mathrm{Z}$ \\
\hline & & & & & $03-12-74$ & 104.9 & -- & $\mathrm{Z}$ \\
\hline & & & & & $11-06-75$ & 105.7 & -- & Z \\
\hline & & & & & $04-06-76$ & 100.4 & -- & $\mathrm{Z}$ \\
\hline & & & & & $11-08-76$ & 111.2 & -- & $\mathrm{Z}$ \\
\hline & & & & & $03-05-77$ & 109.7 & - & $\mathrm{Z}$ \\
\hline & & & & & $11-15-77$ & 114.7 & -- & $\mathrm{Z}$ \\
\hline & & & & & $11-09-78$ & 111.6 & - & $\mathrm{Z}$ \\
\hline & & & & & $04-05-79$ & 105.4 & -- & $\mathrm{Z}$ \\
\hline & & & & & $11-08-79$ & 116.3 & - & $\mathrm{Z}$ \\
\hline & & & & & $04-14-80$ & 111.3 & -- & $\mathrm{Z}$ \\
\hline & & & & & $11-19-80$ & 112.2 & -- & $\mathrm{Z}$ \\
\hline & & & & & $11-00-81$ & 122.6 & -. & $\mathbf{R}$ \\
\hline & & & & & $04-06-82$ & 119.2 & -- & $\mathrm{Z}$ \\
\hline & & & & & $03-30-83$ & 115.4 & -- & $\mathrm{Z}$ \\
\hline \multirow[t]{5}{*}{153} & N21 E54 05BBBB 1 & 394312115551601 & 150 & 5,876 & $11-00-64$ & 25.8 & -- & $\mathrm{R}$ \\
\hline & & & & & $04-07-66$ & 23.43 & -- & $\mathrm{Z}$ \\
\hline & & & & & $11-00-66$ & 28.9 & -- & $\mathbf{R}$ \\
\hline & & & & & $11-00-67$ & 30.1 & -- & $\mathbf{R}$ \\
\hline & & & & & $04-00-68$ & 26.5 & - & $\mathrm{R}$ \\
\hline
\end{tabular}


Appendix. Water-level measurements in selected wells, Diamond Valley, Nevada, 1960-90-Continued

\begin{tabular}{|c|c|c|c|c|c|c|c|c|}
\hline \multirow{2}{*}{\multicolumn{3}{|c|}{$\begin{array}{l}\text { U.S. Geologlcal Survey } \\
\text { site designation }\end{array}$}} & \multirow{3}{*}{$\begin{array}{l}\text { Depth } \\
\text { of } \\
\text { well } \\
\text { (feet) }\end{array}$} & \multirow{3}{*}{$\begin{array}{l}\text { Land- } \\
\text { surface } \\
\text { altitude } \\
\text { (feet above } \\
\text { sea level) }\end{array}$} & \multicolumn{4}{|c|}{ Water-level measurement } \\
\hline & & & & & \multirow[b]{2}{*}{ Date } & \multirow{2}{*}{$\begin{array}{c}\text { Feet } \\
\text { below } \\
\text { land } \\
\text { surface }\end{array}$} & \multirow[b]{2}{*}{ Status } & \multirow[b]{2}{*}{ Method } \\
\hline & $\begin{array}{l}\text { Local } \\
\text { Identification }\end{array}$ & $\begin{array}{l}\text { Standard } \\
\text { Identification }\end{array}$ & & & & & & \\
\hline \multirow[t]{17}{*}{153} & N21 E54 05BBBB1 & (Continued) & & & $08-27-68$ & 33.1 & -- & $\mathrm{Z}$ \\
\hline & & & & & $04-21-69$ & 28.0 & -- & $\mathrm{Z}$ \\
\hline & & & & & $09-11-69$ & 35.3 & $\mathrm{~s}$ & $\mathrm{Z}$ \\
\hline & & & & & $11-28-73$ & 36.2 & -- & $z$ \\
\hline & & & & & $11-06-75$ & 41.6 & -- & $\mathrm{Z}$ \\
\hline & & & & & $04-07-76$ & 36.6 & -- & $\mathrm{Z}$ \\
\hline & & & & & $11-08-76$ & 44.2 & -- & $\mathrm{Z}$ \\
\hline & & & & & $03-05-77$ & 40.8 & -- & $\mathrm{z}$ \\
\hline & & & & & $11-16-77$ & 47.0 & -- & $\mathrm{Z}$ \\
\hline & & & & & $03-30-78$ & 42.30 & -- & $\mathrm{z}$ \\
\hline & & & & & 04-09-79 & 43.3 & -- & $\mathrm{z}$ \\
\hline & & & & & $11-08-79$ & 54.8 & -- & $\mathrm{Z}$ \\
\hline & & & & & $04-15-80$ & 50.0 & -. & $\mathrm{Z}$ \\
\hline & & & & & $11-21-80$ & 55.0 & -- & $\mathrm{z}$ \\
\hline & & & & & $11-00-81$ & 63.6 & -. & $\mathbf{R}$ \\
\hline & & & & & $04-06-82$ & 61.1 & -- & $\mathrm{Z}$ \\
\hline & & & & & $03-31-83$ & 58.3 & -- & $\mathrm{z}$ \\
\hline \multirow[t]{8}{*}{153} & N21 E54 05DCCC1 & 394232115545701 & -- & 5,878 & $04-07-66$ & 24.14 & -- & $S$ \\
\hline & & & & & $10-0 i-73$ & 46.7 & -- & $\mathrm{Z}$ \\
\hline & & & & & $11-03-75$ & 41.2 & -- & $\mathrm{Z}$ \\
\hline & & & & & $03-01-76$ & 42.2 & -- & $\mathrm{Z}$ \\
\hline & & & & & $11-08-76$ & 46.0 & -- & $\mathrm{z}$ \\
\hline & & & & & $11-14-77$ & -- & $\mathbf{P}$ & .- \\
\hline & & & & & $11-08-78$ & 51.6 & -- & $\mathrm{Z}$ \\
\hline & & & & & $11-01-79$ & 59.5 & -- & $\mathrm{Z}$ \\
\hline \multirow[t]{10}{*}{153} & N21 E54 05DCCC1 & 394232115545701 & -- & 5,878 & $11-01-80$ & 60.6 & -- & $\mathrm{z}$ \\
\hline & & & & & $11-01-81$ & 66.8 & -- & $\mathrm{Z}$ \\
\hline & & & & & $11-09-82$ & 67.3 & -- & $\mathrm{z}$ \\
\hline & & & & & $05-01-83$ & 64.9 & -- & $\mathrm{Z}$ \\
\hline & & & & & $11-10-83$ & 66.9 & -- & $\mathrm{z}$ \\
\hline & & & & & $11-01-84$ & 65.3 & -- & $\mathrm{z}$ \\
\hline & & & & & $11-04-86$ & 71.1 & -- & $\mathrm{Z}$ \\
\hline & & & & & $11-18-87$ & 71.6 & - & $\mathrm{Z}$ \\
\hline & & & & & $10-19-88$ & 73.1 & -- & $\mathrm{Z}$ \\
\hline & & & & & $11-02-89$ & 79.7 & -- & $\mathrm{z}$ \\
\hline \multirow[t]{10}{*}{1531} & N21 E54 08CDD1 & 394141115552601 & 203 & 5,895 & $08-29-64$ & 37.0 & -- & -- \\
\hline & & & & & $11-00-65$ & 34.0 & -. & $\mathrm{R}$ \\
\hline & & & & & $04-07-66$ & 33.44 & -- & $\mathrm{Z}$ \\
\hline & & & & & $11-00-67$ & 44.8 & - & $\mathrm{R}$ \\
\hline & & & & & $08-27-68$ & 48.4 & -- & z \\
\hline & & & & & $04-21-69$ & 49.0 & -- & $\mathrm{Z}$ \\
\hline & & & & & $09-11-69$ & 45.9 & $S$ & $\mathrm{Z}$ \\
\hline & & & & & $11-16-71$ & 56.1 & -- & $\mathrm{Z}$ \\
\hline & & & & & $02-24-72$ & 48.2 & -. & $\mathrm{Z}$ \\
\hline & & & & & $08-09-73$ & 54.5 & $\mathrm{~s}$ & $\mathrm{Z}$ \\
\hline
\end{tabular}


Appendix. Water-level measurements in selected wells, Diamond Valley, Nevada, 1960-90—Continued

\begin{tabular}{|c|c|c|c|c|c|c|c|}
\hline \multirow{2}{*}{\multicolumn{2}{|c|}{$\begin{array}{l}\text { U.S. Geological Survey } \\
\text { site designation }\end{array}$}} & \multirow{3}{*}{$\begin{array}{c}\text { Depth } \\
\text { of } \\
\text { well } \\
\text { (feet) }\end{array}$} & \multirow{3}{*}{$\begin{array}{c}\text { Land- } \\
\text { surface } \\
\text { altitude } \\
\text { (feet above } \\
\text { sea level) }\end{array}$} & \multicolumn{4}{|c|}{ Water-level measurement } \\
\hline & & & & \multirow[b]{2}{*}{ Date } & \multirow{2}{*}{$\begin{array}{c}\text { Feet } \\
\text { below } \\
\text { land } \\
\text { surface }\end{array}$} & \multirow[b]{2}{*}{ Status } & \multirow[b]{2}{*}{ Method } \\
\hline $\begin{array}{l}\text { Local } \\
\text { Identiflcation }\end{array}$ & $\begin{array}{l}\text { Standard } \\
\text { Identification }\end{array}$ & & & & & & \\
\hline \multirow{15}{*}{\multicolumn{2}{|c|}{153 N21 E54 08CDD1 (Continued) }} & & & $11-28-73$ & 46.7 & -- & $\mathrm{Z}$ \\
\hline & & & & $11-06-75$ & 49.1 & -- & $\mathrm{Z}$ \\
\hline & & & & $04-07-76$ & 48.7 & -- & $\mathrm{Z}$ \\
\hline & & & & $11-08-76$ & 51.5 & -- & $\mathrm{Z}$ \\
\hline & & & & $03-05-77$ & 56.0 & -- & $\mathrm{Z}$ \\
\hline & & & & $11-16-77$ & 63.7 & -- & $\mathrm{Z}$ \\
\hline & & & & $03-30-78$ & 62.9 & -- & $\mathrm{Z}$ \\
\hline & & & & $11-00-78$ & 66.5 & -- & $\mathbf{R}$ \\
\hline & & & & $04-09-79$ & 64.6 & -- & $\mathrm{Z}$ \\
\hline & & & & $11-08-79$ & 77.0 & $-\cdot$ & $\mathrm{Z}$ \\
\hline & & & & $04-15-80$ & 71.6 & -- & $\mathrm{z}$ \\
\hline & & & & $11-21-80$ & 73.7 & -- & $\mathrm{Z}$ \\
\hline & & & & $11-00-81$ & 78.2 & -- & $\mathbf{R}$ \\
\hline & & & & $04-06-82$ & 67.5 & -- & $\mathrm{Z}$ \\
\hline & & & & $03-31-83$ & 75.5 & -- & $\mathrm{Z}$ \\
\hline \multirow{17}{*}{153 N21 E54 08DBBB1 } & 394206115545801 & -- & 5,880 & $10-01-73$ & 46.7 & -- & $\mathrm{Z}$ \\
\hline & & & & $11-03-75$ & 49.1 & -- & $\mathrm{Z}$ \\
\hline & & & & $03-01-76$ & 48.7 & -- & $\mathrm{Z}$ \\
\hline & & & & $11-08-76$ & 57.2 & -- & $\mathrm{Z}$ \\
\hline & & & & $11-14-77$ & -- & $\mathbf{P}$ & -- \\
\hline & & & & $11-08-78$ & 60.4 & -- & $\mathrm{Z}$ \\
\hline & & & & $11-01-79$ & 65.4 & -- & $\mathrm{Z}$ \\
\hline & & & & $11-01-80$ & 66.8 & -- & $\mathrm{Z}$ \\
\hline & & & & $11-01-81$ & 75.1 & -- & $\mathrm{Z}$ \\
\hline & & & & $11-09-82$ & 79.0 & -- & $\mathrm{z}$ \\
\hline & & & & $05-01-83$ & 76.8 & -- & $\mathrm{Z}$ \\
\hline & & & & $11-10-83$ & 75.6 & -- & $\mathrm{Z}$ \\
\hline & & & & $11-01-84$ & 76.0 & -- & $\mathrm{Z}$ \\
\hline & & & & $11-04-86$ & 81.1 & -- & $\mathrm{Z}$ \\
\hline & & & & $11-18-87$ & 77.4 & -- & $\mathrm{Z}$ \\
\hline & & & & $10-19-88$ & 87.5 & -- & $\mathrm{Z}$ \\
\hline & & & & $11-03-89$ & 80.5 & -- & $\mathrm{Z}$ \\
\hline \multirow[t]{10}{*}{153 N21 E54 16CDCD1 } & 394049115535901 & 240 & 5,980 & $07-31-63$ & 120 & -- & -- \\
\hline & & & & $11-00-63$ & 120.2 & -- & $\mathrm{R}$ \\
\hline & & & & $04-00-64$ & 116.9 & -- & $\mathbf{R}$ \\
\hline & & & & $11-00-65$ & 119.9 & -- & $\mathrm{R}$ \\
\hline & & & & $04-07-66$ & 119.02 & -- & $\mathrm{Z}$ \\
\hline & & & & $11-00-67$ & 135.6 & -- & $\mathbf{R}$ \\
\hline & & & & $08-27-68$ & 138.7 & -- & $\mathrm{Z}$ \\
\hline & & & & $04-21-69$ & 134.8 & -- & $\mathrm{Z}$ \\
\hline & & & & $09-11-69$ & 135.0 & $S$ & $\mathrm{Z}$ \\
\hline & & & & $11-16-71$ & 145.8 & -- & $\mathrm{Z}$ \\
\hline
\end{tabular}


Appendix. Water-level measurements in selected wells, Diamond Valley, Nevada, 1960-90-Continued

\begin{tabular}{|c|c|c|c|c|c|c|c|}
\hline \multirow{2}{*}{\multicolumn{2}{|c|}{$\begin{array}{c}\text { U.S. Geological Survey } \\
\text { site designation }\end{array}$}} & \multirow{3}{*}{$\begin{array}{c}\text { Depth } \\
\text { of } \\
\text { well } \\
\text { (feet) }\end{array}$} & \multirow{3}{*}{$\begin{array}{l}\text { Land- } \\
\text { surface } \\
\text { altitude } \\
\text { (feet above } \\
\text { Sea level) }\end{array}$} & \multicolumn{4}{|c|}{ Water-level measurement } \\
\hline & & & & \multirow[b]{2}{*}{ Date } & \multirow{2}{*}{$\begin{array}{l}\text { Feet } \\
\text { below } \\
\text { land } \\
\text { surface }\end{array}$} & \multirow[b]{2}{*}{ Status } & \multirow[b]{2}{*}{ Method } \\
\hline $\begin{array}{c}\text { Local } \\
\text { identification }\end{array}$ & $\begin{array}{l}\text { Standard } \\
\text { identification }\end{array}$ & & & & & & \\
\hline \multirow[t]{12}{*}{$153 \mathrm{~N} 21 \mathrm{E} 54$ 16CDCD 1} & Continued) & & & $02-24-72$ & 148.1 & -. & $\mathrm{Z}$ \\
\hline & & & & $11-06-75$ & 138.6 & -- & $\mathrm{Z}$ \\
\hline & & & & $11-08-76$ & 142.9 & -- & $\mathrm{Z}$ \\
\hline & & & & $03-05-77$ & 147.5 & -- & $\mathrm{Z}$ \\
\hline & & & & $11-16-77$ & 146.8 & -. & $\mathrm{Z}$ \\
\hline & & & & $03-30-78$ & 141.8 & -- & $z$ \\
\hline & & & & $11-00-78$ & 152.1 & -- & $\mathbf{R}$ \\
\hline & & & & $04-09-79$ & 151.7 & -- & $\mathrm{Z}$ \\
\hline & & & & $11-21-80$ & 159.8 & -- & $\mathrm{z}$ \\
\hline & & & & $11-00-81$ & 160.6 & -- & $\mathrm{R}$ \\
\hline & & & & $04-06-82$ & 157.7 & -- & $\mathrm{z}$ \\
\hline & & & & $03-31-83$ & 157.7 & -- & $\mathrm{Z}$ \\
\hline \multirow[t]{30}{*}{153 N21 E54 17CDDD1 } & 394049115550201 & 240 & 5,920 & $06-24-62$ & 60 & -- & -- \\
\hline & & & & $07-31-62$ & 60.0 & -- & -- \\
\hline & & & & $11-00-63$ & 70.9 & -- & $\mathrm{R}$ \\
\hline & & & & $04-00-64$ & 67.9 & -- & $\mathrm{R}$ \\
\hline & & & & $04-00-65$ & 62.4 & -- & $\mathrm{R}$ \\
\hline & & & & $11-00-65$ & 62.9 & -- & $\mathbf{R}$ \\
\hline & & & & $04-07-66$ & 62.33 & -- & $\mathrm{Z}$ \\
\hline & & & & $04-00-67$ & 64.8 & -- & $\mathrm{R}$ \\
\hline & & & & $11-00-67$ & 85.8 & -- & $\mathbf{R}$ \\
\hline & & & & $04-00-68$ & 77.4 & -- & $\mathrm{R}$ \\
\hline & & & & $08-27-68$ & 84.1 & -- & $\mathrm{z}$ \\
\hline & & & & $04-21-69$ & 84.9 & -- & $\mathrm{Z}$ \\
\hline & & & & $09-10-69$ & 75.8 & $s$ & $\mathrm{Z}$ \\
\hline & & & & $11-16-71$ & 109.8 & -- & Z \\
\hline & & & & $02-24-72$ & 105.8 & -- & $\mathrm{Z}$ \\
\hline & & & & 08-09-73 & 91.6 & $s$ & $\mathrm{z}$ \\
\hline & & & & $11-28-73$ & 105.7 & -- & $\mathrm{Z}$ \\
\hline & & & & $03-12-74$ & 100.8 & -- & $\mathrm{Z}$ \\
\hline & & & & $11-15-75$ & 103.6 & -- & $Z$ \\
\hline & & & & $03-05-77$ & 84.9 & -- & $\mathrm{z}$ \\
\hline & & & & $11-16-77$ & 102.9 & -- & z \\
\hline & & & & $03-30-78$ & 97.4 & -- & $\mathrm{Z}$ \\
\hline & & & & $11-00-78$ & 103.6 & -- & $\mathrm{R}$ \\
\hline & & & & $04-09-79$ & 98.8 & -- & $\mathrm{Z}$ \\
\hline & & & & $11-08-79$ & 109.9 & - & $\mathrm{Z}$ \\
\hline & & & & $04-15-80$ & 101.1 & -. & $\mathrm{z}$ \\
\hline & & & & $11-21-80$ & 98.0 & -- & $\mathrm{Z}$ \\
\hline & & & & $11-00-81$ & 103.8 & -- & $\mathbf{R}$ \\
\hline & & & & $04-06-82$ & 103.5 & -- & $\mathrm{Z}$ \\
\hline & & & & $03-31-83$ & 103.1 & -- & $\mathrm{Z}$ \\
\hline
\end{tabular}


Appendix. Water-level measurements in selected wells, Diamond Valley, Nevada, 1960-90-Continued

\begin{tabular}{|c|c|c|c|c|c|c|c|c|}
\hline \multirow{2}{*}{\multicolumn{3}{|c|}{$\begin{array}{l}\text { U.S. Geological Survey } \\
\text { site designation }\end{array}$}} & \multirow{3}{*}{$\begin{array}{c}\text { Depth } \\
\text { of } \\
\text { well } \\
\text { (feet) }\end{array}$} & \multirow{3}{*}{$\begin{array}{c}\text { Land- } \\
\text { surface } \\
\text { altitude } \\
\text { (feet above } \\
\text { sea level) }\end{array}$} & \multicolumn{4}{|c|}{ Water-level measurement } \\
\hline & & & & & \multirow[b]{2}{*}{ Date } & \multirow{2}{*}{$\begin{array}{c}\text { Feet } \\
\text { below } \\
\text { land } \\
\text { surface }\end{array}$} & \multirow[b]{2}{*}{ Status } & \multirow[b]{2}{*}{ Method } \\
\hline & $\begin{array}{l}\text { Local } \\
\text { Identiflcation }\end{array}$ & $\begin{array}{l}\text { Standard } \\
\text { Identification }\end{array}$ & & & & & & \\
\hline \multirow{30}{*}{\multicolumn{2}{|c|}{153 N21 E54 17DDDD1 }} & 394048115542601 & 200 & 5,949 & $06-15-62$ & 105 & -- & -- \\
\hline & & & & & $04-00-64$ & 90.0 & -- & $\mathbf{R}$ \\
\hline & & & & & $04-00-65$ & 90.6 & -- & $\mathbf{R}$ \\
\hline & & & & & $11-00-65$ & 90.7 & -- & $\mathbf{R}$ \\
\hline & & & & & 04-07-66 & 89.50 & -- & $\mathrm{Z}$ \\
\hline & & & & & $04-00-67$ & 92.9 & -. & $\mathbf{R}$ \\
\hline & & & & & $11-00-67$ & 115.4 & -- & $\mathbf{R}$ \\
\hline & & & & & $04-00-68$ & 97.8 & -- & $\mathbf{R}$ \\
\hline & & & & & $08-27-68$ & 102.8 & -- & $z$ \\
\hline & & & & & $04-21-69$ & 101.4 & -- & $\mathrm{Z}$ \\
\hline & & & & & $09-10-69$ & 107.4 & $S$ & $\mathrm{z}$ \\
\hline & & & & & $11-16-71$ & 120.6 & -- & $\mathrm{Z}$ \\
\hline & & & & & $02-24-72$ & 127.9 & -- & $Z$ \\
\hline & & & & & $08-09-73$ & 119.1 & $\mathrm{~s}$ & $\mathrm{z}$ \\
\hline & & & & & $11-28-73$ & 127.7 & -- & $\mathrm{z}$ \\
\hline & & & & & $04-00-74$ & 121.3 & -- & $\mathrm{Z}$ \\
\hline & & & & & $11-06-75$ & 110.9 & -- & $\mathrm{z}$ \\
\hline & & & & & $04-07-76$ & 106.7 & -- & $\mathrm{Z}$ \\
\hline & & & & & $11-00-76$ & 116.6 & -- & $\mathrm{Z}$ \\
\hline & & & & & $03-05-77$ & 115.4 & -- & $\mathrm{z}$ \\
\hline & & & & & $11-16-77$ & 119.2 & -- & $\mathrm{Z}$ \\
\hline & & & & & $03-30-78$ & 115.6 & -- & $\mathrm{Z}$ \\
\hline & & & & & $11-00-78$ & 120.1 & -- & $\mathbf{R}$ \\
\hline & & & & & 04-09-79 & 118.3 & -- & Z \\
\hline & & & & & $11-08-79$ & 126.0 & -- & $\mathrm{Z}$ \\
\hline & & & & & $04-15-80$ & 122.7 & -- & $\mathrm{Z}$ \\
\hline & & & & & $11-21-80$ & 127.4 & -- & $\mathrm{Z}$ \\
\hline & & & & & $11-00-81$ & 133.3 & -- & $\mathbf{R}$ \\
\hline & & & & & $04-06-82$ & 129.0 & -- & Z \\
\hline & & & & & $03-31-83$ & 130.1 & -- & $\mathrm{Z}$ \\
\hline \multirow[t]{15}{*}{153} & N21 E54 20BACC1 & 394037115551401 & -- & 5,921 & $04-07-66$ & 67.98 & -- & $\mathrm{Z}$ \\
\hline & & & & & $08-27-68$ & 87.6 & -- & Z \\
\hline & & & & & $04-21-69$ & 80.6 & -- & Z \\
\hline & & & & & $09-11-69$ & 79.2 & $S$ & Z \\
\hline & & & & & $11-16-71$ & 78.8 & -- & Z \\
\hline & & & & & $02-24-72$ & 78.3 & .- & $\mathrm{Z}$ \\
\hline & & & & & $08-09-73$ & 97.2 & $S$ & $\bar{Z}$ \\
\hline & & & & & $11-00-73$ & 82.2 & -- & Z \\
\hline & & & & & $11-06-75$ & 86.2 & -- & Z \\
\hline & & & & & $04-07-76$ & 84.0 & -- & $\mathrm{z}$ \\
\hline & & & & & $11-08-76$ & 95.2 & -- & Z \\
\hline & & & & & $03-05-77$ & 94.2 & -- & Z \\
\hline & & & & & $11-16-77$ & 99.3 & -- & Z \\
\hline & & & & & $03-30-78$ & 91.2 & -- & Z \\
\hline & & & & & $11-00-78$ & 99.4 & -- & $\mathrm{R}$ \\
\hline
\end{tabular}


Appendix. Water-level measurements in selected wells, Diamond Valley, Nevada, 1960-90-Continued

\begin{tabular}{|c|c|c|c|c|c|c|c|c|}
\hline \multirow{2}{*}{\multicolumn{3}{|c|}{$\begin{array}{c}\text { U.S. Geological Survey } \\
\text { site designation }\end{array}$}} & \multirow{3}{*}{$\begin{array}{l}\text { Depth } \\
\text { of } \\
\text { well } \\
\text { (feet) }\end{array}$} & \multirow{3}{*}{$\begin{array}{l}\text { Land- } \\
\text { surface } \\
\text { altitude } \\
\text { (feet above } \\
\text { sea level) }\end{array}$} & \multicolumn{4}{|c|}{ Water-level measurement } \\
\hline & & & & & \multirow[b]{2}{*}{ Date } & \multirow{2}{*}{$\begin{array}{c}\text { Feet } \\
\text { below } \\
\text { land } \\
\text { surface }\end{array}$} & \multirow[b]{2}{*}{ Status } & \multirow[b]{2}{*}{ Method } \\
\hline & $\begin{array}{l}\text { Local } \\
\text { Identification }\end{array}$ & $\begin{array}{l}\text { Standard } \\
\text { ident|flcation }\end{array}$ & & & & & & \\
\hline \multirow[t]{5}{*}{153} & N21 E54 20BACC1 & (Continued) & & & 04-09-79 & 95.2 & -- & $\mathrm{Z}$ \\
\hline & & & & & $11-08-79$ & 102.1 & -- & $\mathrm{Z}$ \\
\hline & & & & & $04-15-80$ & 98.0 & -- & $\mathrm{Z}$ \\
\hline & & & & & $11-21-80$ & 103.2 & -- & $z$ \\
\hline & & & & & $03-31-83$ & 106.7 & -- & $\mathrm{Z}$ \\
\hline \multirow[t]{16}{*}{153} & N21 E54 20CCCC1 & 393958115552701 & 230 & 5,932 & $04-07-66$ & 83.05 & -- & $s$ \\
\hline & & & & & $11-03-75$ & 96.0 & -- & $\mathrm{Z}$ \\
\hline & & & & & $03-01-76$ & 93.5 & -- & $\mathrm{z}$ \\
\hline & & & & & $11-08-76$ & 99.1 & -- & $\mathrm{Z}$ \\
\hline & & & & & $11-14-77$ & 101.3 & -- & $\bar{z}$ \\
\hline & & & & & $11-08-78$ & 104.5 & -- & $\mathrm{Z}$ \\
\hline & & & & & $11-01-79$ & 108.0 & -- & $\mathrm{Z}$ \\
\hline & & & & & $11-01-80$ & 110.0 & -- & $\mathrm{Z}$ \\
\hline & & & & & $11-01-81$ & 126.5 & .- & $\bar{z}$ \\
\hline & & & & & $11-09-82$ & 123.1 & -- & $\mathrm{z}$ \\
\hline & & & & & $05-01-83$ & 115.8 & -- & $\mathrm{Z}$ \\
\hline & & & & & $11-10-83$ & 122.0 & -- & Z \\
\hline & & & & & $11-09-84$ & 119.7 & -- & Z \\
\hline & & & & & $11-12-86$ & 119.4 & -- & $\mathrm{Z}$ \\
\hline & & & & & $11-03-89$ & 124.2 & -- & Z \\
\hline & & & & & $10-18-90$ & 124.7 & -- & $\mathrm{Z}$ \\
\hline \multirow[t]{9}{*}{153} & N21 HE52 01BC 1 & 394342114385401 & 70 & 5,888 & $10-21-65$ & 50.20 & -- & $\mathrm{s}$ \\
\hline & & & & & $04-08-66$ & 48.78 & -- & $\mathrm{s}$ \\
\hline & & & & & $04-05-77$ & 58.11 & .. & $\mathrm{S}$ \\
\hline & & & & & $04-12-78$ & 59.34 & -- & $\mathrm{s}$ \\
\hline & & & & & 03-20-79 & 60.81 & -- & $\mathrm{s}$ \\
\hline & & & & & $03-19-80$ & 62.55 & -- & $\mathrm{s}$ \\
\hline & & & & & $03-20-81$ & 63.95 & -- & $\mathrm{s}$ \\
\hline & & & & & $03-16-82$ & 65.28 & -- & $S$ \\
\hline & & & & & $02-09-87$ & -- & & -- \\
\hline \multirow[t]{4}{*}{153} & N21HE52 01BC 2 & 394342114385402 & 160 & 5,888 & $02-09-87$ & 72.05 & -- & $\mathrm{s}$ \\
\hline & & & & & $03-18-88$ & 73.02 & -- & $\mathrm{s}$ \\
\hline & & & & & $04-20-89$ & $\cdots$ & $\mathrm{P}$ & -- \\
\hline & & & & & $05-24-90$ & 74.02 & -- & $\mathrm{s}$ \\
\hline \multirow[t]{10}{*}{153} & N21HE54 32DCCC1 & 394327115545301 & 242 & 5,867 & $04-15-62$ & 12 & -- & .. \\
\hline & & & & & $11-00-63$ & 22.6 & -- & $\mathbf{R}$ \\
\hline & & & & & $04-00-64$ & 23.5 & -- & $\mathbf{R}$ \\
\hline & & & & & $04-00-65$ & 23.2 & -- & $\mathbf{R}$ \\
\hline & & & & & $08-27-68$ & 33.2 & -- & $\mathrm{Z}$ \\
\hline & & & & & $04-21-69$ & 26.2 & -- & $\mathrm{z}$ \\
\hline & & & & & $08-12-69$ & 29.9 & -- & $\bar{z}$ \\
\hline & & & & & $03-11-74$ & 35.0 & -. & $\mathrm{Z}$ \\
\hline & & & & & $11-06-75$ & 35.7 & -- & $\mathrm{Z}$ \\
\hline & & & & & $04-00-76$ & 32.1 & -. & Z \\
\hline
\end{tabular}


Appendix. Water-level measurements in selected wells, Diamond Valley, Nevada, 1960-90—Continued

\begin{tabular}{|c|c|c|c|c|c|c|c|}
\hline & & & & & Vater-level & asureme & \\
\hline site desi & nation & of & surface & & Feet & & \\
\hline $\begin{array}{c}\text { Local } \\
\text { Identification }\end{array}$ & $\begin{array}{l}\text { Standard } \\
\text { Identification }\end{array}$ & (feet) & $\begin{array}{l}\text { (feet above } \\
\text { sea level) }\end{array}$ & Date & $\begin{array}{l}\text { land } \\
\text { surface }\end{array}$ & Status & Method \\
\hline 153 N21HE54 32DCCC1 & Continued) & & & $11-08-76$ & 38.5 & -- & $\mathrm{Z}$ \\
\hline & & & & $03-05-77$ & 33.8 & -- & $\mathrm{Z}$ \\
\hline & & & & $11-16-77$ & 46.2 & -- & $\mathrm{Z}$ \\
\hline & & & & $03-30-78$ & 40.7 & -- & $z$ \\
\hline & & & & $11-14-78$ & 49.2 & -- & $\mathrm{z}$ \\
\hline & & & & $04-12-79$ & 44.0 & -- & $\mathrm{z}$ \\
\hline & & & & $11-07-79$ & 49.2 & -- & $\mathrm{Z}$ \\
\hline & & & & $04-17-80$ & 42.1 & -- & $\mathrm{z}$ \\
\hline & & & & $11-21-80$ & 49.2 & -- & $\mathrm{Z}$ \\
\hline & & & & $11-09-82$ & 56.3 & -- & $\mathrm{Z}$ \\
\hline & & & & $03-31-83$ & 53.7 & -- & $\mathrm{Z}$ \\
\hline 153 N21HE54 33DDDD1 & 394326115531701 & 102 & 5,880 & $01-10-62$ & 21.58 & -- & -- \\
\hline & & & & $04-26-66$ & 32.08 & -- & $\mathrm{R}$ \\
\hline & & & & $11-00-67$ & 39.7 & -- & $\mathrm{R}$ \\
\hline & & & & $04-00-68$ & 28.3 & -- & $\mathrm{R}$ \\
\hline & & & & $08-27-68$ & 46.0 & -- & $\mathrm{Z}$ \\
\hline & & & & $04-21-69$ & 39.0 & -- & $\mathrm{z}$ \\
\hline & & & & $09-12-69$ & 43.9 & - & $\mathrm{R}$ \\
\hline & & & & $11-16-71$ & 42.7 & - & $\mathrm{Z}$ \\
\hline & & & & $02-24-72$ & 41.1 & -- & $\mathrm{Z}$ \\
\hline & & & & $08-09-73$ & 45.0 & -- & Z \\
\hline & & & & $11-28-73$ & 44.0 & -- & $\mathrm{Z}$ \\
\hline & & & & $03-12-74$ & 43.8 & -- & $\mathrm{z}$ \\
\hline & & & & $11-14-75$ & 59.1 & -- & $z$ \\
\hline & & & & $03-05-77$ & 50.8 & -- & $\mathrm{Z}$ \\
\hline & & & & $11-16-77$ & 53.0 & -- & $z$ \\
\hline & & & & $03-30-78$ & 54.1 & -- & $\mathrm{z}$ \\
\hline & & & & $11-00-78$ & 59.1 & -- & $\mathbf{R}$ \\
\hline & & & & $04-12-79$ & 55.0 & -- & $\mathrm{Z}$ \\
\hline & & & & $11-07-79$ & 62.3 & -- & $Z$ \\
\hline & & & & $04-17-80$ & 58.2 & -- & $\mathrm{Z}$ \\
\hline & & & & $11-21-80$ & 66.1 & -- & $\mathrm{z}$ \\
\hline & & & & $11-09-82$ & 74.7 & -- & $\mathrm{Z}$ \\
\hline & & & & $03-31-83$ & 69.2 & -- & Z \\
\hline 153 N22 E54 05CDBB 1 & 394839115550801 & 210 & 5,836 & $04-07-66$ & 3.88 & -- & $\mathrm{s}$ \\
\hline & & & & $10-01-73$ & 11.9 & -- & $\mathrm{Z}$ \\
\hline & & & & $11-03-75$ & 15.7 & -- & $\mathrm{Z}$ \\
\hline & & & & $03-01-76$ & 9.8 & -- & $\mathrm{Z}$ \\
\hline & & & & $11-08-76$ & 17.4 & -- & $\mathrm{Z}$ \\
\hline & & & & $11-14-77$ & 21.0 & -- & $\mathrm{z}$ \\
\hline & & & & $11-08-78$ & 22.1 & -- & Z \\
\hline & & & & $11-01-79$ & 29.7 & -- & $\mathrm{Z}$ \\
\hline & & & & $11-01-80$ & 26.8 & -- & $\mathrm{Z}$ \\
\hline & & & & $11-01-81$ & 39.9 & -- & $\bar{Z}$ \\
\hline
\end{tabular}


Appendix. Water-level measurements in selected wells, Diamond Valley, Nevada, 1960-90-Continued

\begin{tabular}{|c|c|c|c|c|c|c|c|c|}
\hline \multirow{2}{*}{\multicolumn{3}{|c|}{$\begin{array}{l}\text { U.S. Geological Survey } \\
\text { site designation }\end{array}$}} & \multirow{3}{*}{$\begin{array}{c}\text { Depth } \\
\text { of } \\
\text { well } \\
\text { (feet) }\end{array}$} & \multirow{3}{*}{$\begin{array}{c}\text { Land- } \\
\text { surface } \\
\text { altitude } \\
\text { (feet above } \\
\text { sea level) }\end{array}$} & \multicolumn{4}{|c|}{ Water-level measurement } \\
\hline & & & & & \multirow[b]{2}{*}{ Date } & \multirow{2}{*}{$\begin{array}{c}\text { Feet } \\
\text { below } \\
\text { land } \\
\text { surface }\end{array}$} & \multirow[b]{2}{*}{ Status } & \multirow[b]{2}{*}{ Method } \\
\hline & $\begin{array}{c}\text { Local } \\
\text { Identification }\end{array}$ & $\begin{array}{c}\text { Standard } \\
\text { identlflcatlon }\end{array}$ & & & & & & \\
\hline \multirow{8}{*}{\multicolumn{3}{|c|}{153 N22 E54 05CDBB1 (Continued) }} & & & $11-09-82$ & 32.9 & -- & $\mathrm{Z}$ \\
\hline & & & & & $05-01-83$ & 23.2 & -- & $\mathrm{Z}$ \\
\hline & & & & & $10-07-83$ & 36.0 & -- & $\mathrm{Z}$ \\
\hline & & & & & $11-01-84$ & 35.2 & -- & $\mathrm{Z}$ \\
\hline & & & & & $11-04-86$ & 36.8 & $-\cdot$ & $\mathrm{Z}$ \\
\hline & & & & & $11-12-87$ & 40.0 & -- & $\mathrm{Z}$ \\
\hline & & & & & $10-17-88$ & 48.3 & -- & $\mathrm{Z}$ \\
\hline & & & & & $11-02-89$ & 40.8 & -- & $\mathrm{Z}$ \\
\hline \multirow{15}{*}{\multicolumn{2}{|c|}{153 N22 E54 05DDBB 1}} & 394833115542001 & 240 & 5,835 & $04-07-66$ & 3.58 & -- & $\mathrm{S}$ \\
\hline & & & & & $11-03-75$ & 12.6 & -- & $\mathrm{Z}$ \\
\hline & & & & & $03-01-76$ & 9.6 & -- & Z \\
\hline & & & & & $11-08-76$ & 17.6 & -- & $\mathrm{Z}$ \\
\hline & & & & & $11-14-77$ & 20.0 & - & $\mathrm{Z}$ \\
\hline & & & & & $11-08-78$ & 23.0 & - & $\mathbf{Z}$ \\
\hline & & & & & $11-01-79$ & 30.8 & -- & $\mathrm{Z}$ \\
\hline & & & & & $11-01-80$ & 27.0 & -- & $\mathbf{Z}$ \\
\hline & & & & & $11-01-81$ & 34.9 & -- & $\mathbf{Z}$ \\
\hline & & & & & $11-09-82$ & 29.8 & -- & $\mathrm{Z}$ \\
\hline & & & & & $05-01-83$ & 20.0 & -- & $\mathrm{Z}$ \\
\hline & & & & & $11-07-83$ & 30.2 & -- & $\mathrm{Z}$ \\
\hline & & & & & $11-01-84$ & 30.8 & -- & $\mathrm{Z}$ \\
\hline & & & & & $11-04-86$ & 35.4 & - & $\mathrm{Z}$ \\
\hline & & & & & $11-12-87$ & 38.7 & -- & $\mathrm{Z}$ \\
\hline \multirow{12}{*}{\multicolumn{2}{|c|}{$153 \mathrm{~N} 22 \mathrm{E} 5406 \mathrm{CCCCl}$}} & 394835115561801 & 250 & 5,840 & $04-07-66$ & 7.82 & -- & $S$ \\
\hline & & & & & $10-01-73$ & 15.3 & -- & $\mathrm{Z}$ \\
\hline & & & & & $11-03-75$ & 18.5 & $-\cdot$ & $\mathrm{Z}$ \\
\hline & & & & & $03-01-76$ & 15.2 & -- & $\mathrm{Z}$ \\
\hline & & & & & $11-08-76$ & 20.2 & - & $\mathrm{Z}$ \\
\hline & & & & & $11-14-77$ & 23.1 & -- & $\mathrm{Z}$ \\
\hline & & & & & $11-08-78$ & 24.9 & -- & $\mathrm{Z}$ \\
\hline & & & & & $11-01-79$ & 33.6 & -- & $\mathrm{Z}$ \\
\hline & & & & & $11-04-86$ & 36.9 & -- & $\mathrm{Z}$ \\
\hline & & & & & $11-12-87$ & 39.7 & -- & $\mathbf{Z}$ \\
\hline & & & & & $10-17-88$ & 47.6 & -- & $\mathrm{Z}$ \\
\hline & & & & & $11-03-89$ & 43.9 & -- & $\mathrm{Z}$ \\
\hline \multirow{10}{*}{\multicolumn{2}{|c|}{153 N22 E54 07DDCD2 }} & 394743115554302 & 107 & 5,843 & $11-00-63$ & 13.0 & - & $\mathrm{Z}$ \\
\hline & & & & & $11-00-64$ & 15.5 & -- & $\mathrm{Z}$ \\
\hline & & & & & $11-07-75$ & 27.1 & -- & $\mathrm{Z}$ \\
\hline & & & & & $04-08-76$ & 17.6 & -- & $\mathrm{Z}$ \\
\hline & & & & & $11-08-76$ & 25.1 & -- & $\mathrm{Z}$ \\
\hline & & & & & $03-05-77$ & 19.2 & -- & $\mathrm{Z}$ \\
\hline & & & & & $11-17-77$ & 23.1 & -- & $\mathrm{Z}$ \\
\hline & & & & & $04-04-78$ & 21.1 & -- & $\mathbf{Z}$ \\
\hline & & & & & $11-14-78$ & 26.2 & -- & $\mathrm{Z}$ \\
\hline & & & & & $11-07-79$ & 29.4 & -- & $\mathbf{Z}$ \\
\hline
\end{tabular}


Appendix. Water-level measurements in selected wells, Diamond Valley, Nevada, 1960-90-Continued

\begin{tabular}{|c|c|c|c|c|c|c|c|}
\hline \multirow{2}{*}{\multicolumn{2}{|c|}{$\begin{array}{l}\text { U.S. Geological Survey } \\
\text { site designation }\end{array}$}} & \multirow{3}{*}{$\begin{array}{c}\text { Depth } \\
\text { of } \\
\text { well } \\
\text { (feet) }\end{array}$} & \multirow{3}{*}{$\begin{array}{c}\text { Land- } \\
\text { surface } \\
\text { altitude } \\
\text { (feet above } \\
\text { sea level) }\end{array}$} & \multicolumn{4}{|c|}{ Water-level measurement } \\
\hline & & & & \multirow[b]{2}{*}{ Date } & \multirow{2}{*}{$\begin{array}{c}\text { Feet } \\
\text { below } \\
\text { land } \\
\text { surface }\end{array}$} & \multirow[b]{2}{*}{ Status } & \multirow[b]{2}{*}{ Method } \\
\hline $\begin{array}{l}\text { Local } \\
\text { identification }\end{array}$ & $\begin{array}{c}\text { Standard } \\
\text { Identification }\end{array}$ & & & & & & \\
\hline \multirow{4}{*}{\multicolumn{2}{|c|}{ I53 N22 E54 07DDCD2 (Continued) }} & & & $04-17-80$ & 23.4 & -- & $\mathrm{Z}$ \\
\hline & & & & $12-01-80$ & 29.4 & -- & $Z$ \\
\hline & & & & $11-09-82$ & 32.0 & -- & $\mathrm{Z}$ \\
\hline & & & & $04-01-83$ & 25.3 & -- & $\mathrm{Z}$ \\
\hline \multirow[t]{29}{*}{153 N22 E54 08CDCD 1} & 394743115550601 & -- & 5,843 & $10-16-63$ & 11.97 & $\cdots$ & $\mathrm{Z}$ \\
\hline & & & & $11-00-63$ & 12.9 & -- & $\mathrm{Z}$ \\
\hline & & & & $04-00-64$ & 11.0 & $\cdots$ & $Z$ \\
\hline & & & & $09-16-64$ & 14.78 & -- & $\mathrm{Z}$ \\
\hline & & & & $11-00-64$ & 14.8 & - & $\mathrm{Z}$ \\
\hline & & & & $04-05-65$ & 9.2 & -- & $\mathrm{Z}$ \\
\hline & & & & $10-20-65$ & 11.20 & -- & $\mathrm{Z}$ \\
\hline & & & & $04-07-66$ & 8.06 & -- & $\mathrm{Z}$ \\
\hline & & & & $04-00-67$ & 10.5 & -- & Z \\
\hline & & & & $11-00-67$ & 17.1 & -- & $\mathrm{Z}$ \\
\hline & & & & $04-00-68$ & 11.1 & -- & $\mathrm{Z}$ \\
\hline & & & & $08-28-68$ & 19.7 & -- & $\mathrm{Z}$ \\
\hline & & & & $04-23-69$ & 10.8 & -. & $\mathrm{Z}$ \\
\hline & & & & $09-17-69$ & 19.5 & -- & $Z$ \\
\hline & & & & $|1-16-7|$ & 13.9 & -- & $\mathrm{Z}$ \\
\hline & & & & $08-10-73$ & 30.0 & -- & $\mathrm{Z}$ \\
\hline & & & & $11-29-73$ & 17.7 & -- & Z \\
\hline & & & & $11-07-75$ & 20.1 & -- & $\mathrm{Z}$ \\
\hline & & & & $04-08-76$ & 14.8 & -- & $\mathrm{Z}$ \\
\hline & & & & $11-08-76$ & 21.6 & -- & $\mathrm{Z}$ \\
\hline & & & & $03-05-77$ & 15.0 & -- & $\mathrm{Z}$ \\
\hline & & & & $11-17-77$ & 25.2 & -- & $\mathbf{Z}$ \\
\hline & & & & $04-04-78$ & 19.0 & -- & Z \\
\hline & & & & $11-14-78$ & 26.4 & -- & $\mathrm{Z}$ \\
\hline & & & & $11-07-79$ & 32.9 & -- & $\mathrm{Z}$ \\
\hline & & & & $04-17-80$ & 26.2 & -- & $Z$ \\
\hline & & & & $12-01-80$ & 32.6 & -- & $\mathrm{Z}$ \\
\hline & & & & $11-09-82$ & 35.4 & -- & $\bar{Z}$ \\
\hline & & & & $04-01-83$ & 26.8 & -- & Z \\
\hline \multirow[t]{10}{*}{153 N22 E54 18CADD1 } & 394703115560401 & 258 & 5,848 & $09-23-66$ & 13 & -- & -- \\
\hline & & & & $04-00-68$ & 14.9 & -- & $\mathrm{Z}$ \\
\hline & & & & $08-28-68$ & 20.9 & -- & $\mathrm{Z}$ \\
\hline & & & & $04-23-69$ & 14.8 & -- & $\mathrm{Z}$ \\
\hline & & & & $11-00-69$ & 21.0 & -- & $\mathrm{Z}$ \\
\hline & & & & $|1-| 6-7 \mid$ & 15.2 & -- & $\mathrm{Z}$ \\
\hline & & & & $10-01-73$ & 15.8 & -- & Z \\
\hline & & & & $11-03-75$ & 24.2 & -. & $Z$ \\
\hline & & & & $03-01-76$ & 19.4 & -- & $\mathrm{Z}$ \\
\hline & & & & $11-08-76$ & 26.1 & -- & Z \\
\hline
\end{tabular}


Appendix. Water-level measurements in selected wells, Diamond Valley, Nevada, 1960-90-Continued

\begin{tabular}{|c|c|c|c|c|c|c|c|c|}
\hline \multirow{2}{*}{\multicolumn{3}{|c|}{$\begin{array}{l}\text { U.S. Geological Survey } \\
\text { site designation }\end{array}$}} & \multirow{3}{*}{$\begin{array}{c}\text { Depth } \\
\text { of } \\
\text { well } \\
\text { (feet) }\end{array}$} & \multirow{3}{*}{$\begin{array}{c}\text { Land- } \\
\text { surface } \\
\text { altitude } \\
\text { (feet above } \\
\text { sea level) }\end{array}$} & \multicolumn{4}{|c|}{ Water-level measurement } \\
\hline & & & & & \multirow[b]{2}{*}{ Date } & \multirow{2}{*}{$\begin{array}{c}\text { Feet } \\
\text { below } \\
\text { land } \\
\text { surface }\end{array}$} & \multirow[b]{2}{*}{ Status } & \multirow[b]{2}{*}{ Method } \\
\hline & $\begin{array}{c}\text { Local } \\
\text { Identification }\end{array}$ & $\begin{array}{c}\text { Standard } \\
\text { Identification }\end{array}$ & & & & & & \\
\hline \multirow{17}{*}{\multicolumn{2}{|c|}{153 N22 E54 18CADD1 }} & ontinued) & & & $04-00-77$ & 22.1 & -- & Z \\
\hline & & & & & $11-14-77$ & 29.0 & -- & $\mathrm{Z}$ \\
\hline & & & & & $04-04-78$ & 24.0 & -- & $\mathrm{Z}$ \\
\hline & & & & & $11-08-78$ & 31.2 & -- & $\mathrm{Z}$ \\
\hline & & & & & $11-01-79$ & 35.8 & -- & $\mathrm{Z}$ \\
\hline & & & & & $04-17-80$ & 27.8 & -- & $\mathrm{Z}$ \\
\hline & & & & & $11-01-80$ & 34.3 & -- & $\mathrm{Z}$ \\
\hline & & & & & $11-01-81$ & 42.5 & -- & $\mathrm{Z}$ \\
\hline & & & & & $11-09-82$ & 38.8 & -- & $\mathrm{Z}$ \\
\hline & & & & & $05-01-83$ & 36.8 & -- & $\mathrm{Z}$ \\
\hline & & & & & $11-07-83$ & 39.9 & -- & $\mathrm{Z}$ \\
\hline & & & & & $11-01-84$ & 40.6 & -- & Z \\
\hline & & & & & $12-03-85$ & 39.4 & -- & Z \\
\hline & & & & & $11-04-86$ & 43.5 & -- & Z \\
\hline & & & & & $11-12-87$ & 44.5 & -- & $\mathrm{Z}$ \\
\hline & & & & & $10-17-88$ & 54.1 & -- & $\mathrm{Z}$ \\
\hline & & & & & $11-03-89$ & 49.5 & -- & $\mathrm{Z}$ \\
\hline \multirow[t]{22}{*}{153} & N22 E54 18DAACl & 394709115554301 & 200 & 5,850 & $11-00-61$ & 15.9 & -- & $\mathrm{Z}$ \\
\hline & & & & & $11-00-63$ & 15.2 & -- & Z \\
\hline & & & & & $11-00-64$ & 17.3 & -- & Z \\
\hline & & & & & $03-00-65$ & 12.1 & -- & $\mathrm{Z}$ \\
\hline & & & & & $03-00-66$ & 12.4 & -- & $\mathrm{Z}$ \\
\hline & & & & & $04-07-66$ & 12.35 &.- & $S$ \\
\hline & & & & & $03-00-67$ & 14.0 & -- & $\mathrm{Z}$ \\
\hline & & & & & $03-00-68$ & 15.5 & $\cdots$ & $\bar{Z}$ \\
\hline & & & & & $11-00-68$ & 18.0 & -- & $\mathrm{Z}$ \\
\hline & & & & & $03-00-69$ & 14.4 & - & $\mathrm{Z}$ \\
\hline & & & & & $11-00-69$ & 18.1 & -. & $\mathbf{Z}$ \\
\hline & & & & & $11-00-71$ & 14.5 & -- & Z \\
\hline & & & & & $11-00-73$ & 14.7 & $\cdots$ & $\mathrm{Z}$ \\
\hline & & & & & $11-00-75$ & 18.6 & - & Z \\
\hline & & & & & $03-00-76$ & 16.3 & -- & $\mathrm{Z}$ \\
\hline & & & & & $11-00-76$ & 21.7 & - & $\mathrm{Z}$ \\
\hline & & & & & $03-00-77$ & 14.4 & -- & $\mathrm{Z}$ \\
\hline & & & & & $11-00-77$ & 22.4 & -- & $\mathrm{Z}$ \\
\hline & & & & & $03-00-78$ & 18.6 & - & $\mathrm{Z}$ \\
\hline & & & & & $11-00-78$ & 24.2 & -- & $\mathrm{Z}$ \\
\hline & & & & & $11-00-79$ & 31.4 & -- & Z \\
\hline & & & & & $11-00-81$ & 41.3 & -- & $Z$ \\
\hline \multirow[t]{5}{*}{153} & N22 E54 22BDAA1 & 394611115525101 & 200 & 5,863 & $04-06-66$ & 20.42 & - & $S$ \\
\hline & & & & & $10-01-73$ & 26.1 & -- & $Z$ \\
\hline & & & & & $11-03-75$ & 31.1 & -- & $\mathrm{Z}$ \\
\hline & & & & & $03-01-76$ & 28.4 & -- & $\mathrm{Z}$ \\
\hline & & & & & $11-08-76$ & 36.1 & -- & $\mathrm{Z}$ \\
\hline
\end{tabular}


Appendix. Water-level measurements in selected wells, Diamond Valley, Nevada, 1960-90-Continued

\begin{tabular}{|c|c|c|c|c|c|c|c|c|}
\hline \multirow{2}{*}{\multicolumn{3}{|c|}{$\begin{array}{l}\text { U.S. Geological Survey } \\
\text { site designation }\end{array}$}} & \multirow{3}{*}{$\begin{array}{c}\text { Depth } \\
\text { of } \\
\text { well } \\
\text { (feet) }\end{array}$} & \multirow{3}{*}{$\begin{array}{l}\text { Land- } \\
\text { surface } \\
\text { altitude } \\
\text { (feet above } \\
\text { sea level) }\end{array}$} & \multicolumn{4}{|c|}{ Water-level measurement } \\
\hline & & & & & \multirow[b]{2}{*}{ Date } & \multirow{2}{*}{$\begin{array}{c}\text { Feet } \\
\text { below } \\
\text { land } \\
\text { surface }\end{array}$} & \multirow[b]{2}{*}{ Status } & \multirow[b]{2}{*}{ Method } \\
\hline & $\begin{array}{c}\text { Local } \\
\text { identification }\end{array}$ & $\begin{array}{c}\text { Standard } \\
\text { identification }\end{array}$ & & & & & & \\
\hline \multirow{12}{*}{\multicolumn{3}{|c|}{ i 53 N22 E54 22BDAA1 (Continued) }} & & & $11-14-77$ & 40.5 & -- & Z \\
\hline & & & & & $11-08-78$ & 42.6 & -- & Z \\
\hline & & & & & $11-01-79$ & 48.9 & -- & Z \\
\hline & & & & & $11-01-80$ & 50.5 & -- & Z \\
\hline & & & & & $|1-01-8|$ & 51.1 & - & $\mathbf{Z}$ \\
\hline & & & & & $11-09-82$ & 50.8 & - & Z \\
\hline & & & & & $05-01-83$ & 46.9 & -- & $\mathrm{Z}$ \\
\hline & & & & & $11-07-83$ & 47.5 & - & $\mathrm{Z}$ \\
\hline & & & & & $11-01-84$ & 44.1 & -- & $\mathrm{Z}$ \\
\hline & & & & & $11-12-87$ & 56.9 & -- & $\mathrm{Z}$ \\
\hline & & & & & $10-17-88$ & 60.8 & - & $\mathrm{Z}$ \\
\hline & & & & & $11-02-89$ & 63.7 & -- & $\mathrm{Z}$ \\
\hline \multirow[t]{14}{*}{153} & N22 E54 22CCDD1 & 394558115525801 & 120 & 5,858 & $03-15-62$ & 8.0 & -- &.- \\
\hline & & & & & $11-00-65$ & 14.5 & -- & $\mathrm{Z}$ \\
\hline & & & & & $04-07-66$ & 12.58 & -- & $\mathrm{Z}$ \\
\hline & & & & & $03-12-74$ & 19.2 & -- & Z \\
\hline & & & & & $11-06-75$ & 24.6 & -- & $Z$ \\
\hline & & & & & $04-08-76$ & 21.6 & -- & $\mathrm{Z}$ \\
\hline & & & & & $11-08-76$ & 28.7 & -- & $\mathrm{Z}$ \\
\hline & & & & & $03-05-77$ & 25.2 & -- & $\mathrm{Z}$ \\
\hline & & & & & $04-04-78$ & 28.9 & -- & $\mathrm{Z}$ \\
\hline & & & & & $11-14-78$ & 34.1 & -- & $\mathrm{Z}$ \\
\hline & & & & & $11-07-79$ & 37.8 & -- & $\mathrm{Z}$ \\
\hline & & & & & $04-17-80$ & 32.8 & -- & $\mathrm{Z}$ \\
\hline & & & & & $11-21-80$ & 37.6 & -- & $\mathrm{Z}$ \\
\hline & & & & & $04-01-83$ & 45.6 & -- & $\mathrm{Z}$ \\
\hline \multirow[t]{20}{*}{153} & N22 E54 27CAAB & 394520115524001 & 94 & 5,858 & $08-11-49$ & 5.49 & -- & -- \\
\hline & & & & & $03-15-51$ & 8.39 & -- & -- \\
\hline & & & & & $09-11-51$ & 8.18 & -- & -- \\
\hline & & & & & $10-01-52$ & 7.33 & -- & $S$ \\
\hline & & & & & $03-03-53$ & 8.55 & -- & -- \\
\hline & & & & & $09.15-53$ & 10.04 & -- & -- \\
\hline & & & & & $03-10-54$ & 10.15 & -- & -- \\
\hline & & & & & $09-17-54$ & 10.87 & -- & -- \\
\hline & & & & & $08-29-55$ & 11.84 & -- & -- \\
\hline & & & & & $09-06-57$ & 12.44 & -- & $\cdots$ \\
\hline & & & & & $09-22-61$ & 12.68 & -- & -- \\
\hline & & & & & $03-25-62$ & 11.08 & -- & -- \\
\hline & & & & & $10-14-62$ & 14.74 & -- & -- \\
\hline & & & & & $03-25-63$ & 12.21 & -- & -- \\
\hline & & & & & $09-10-63$ & 19.27 & -- & -- \\
\hline & & & & & $03-24-64$ & 17.01 & -. & - \\
\hline & & & & & $09-21-65$ & 17.49 & -- & -- \\
\hline & & & & & $04-07-66$ & 15.52 & -- & $S$ \\
\hline & & & & & $03-23-67$ & 19.69 & -. & $S$ \\
\hline & & & & & $03-20-68$ & 21.00 & -- & $S$ \\
\hline
\end{tabular}


Appendix. Water-level measurements in selected wells, Diamond Valley, Nevada, 1960-90-Continued

\begin{tabular}{|c|c|c|c|c|c|c|c|c|}
\hline \multirow{2}{*}{\multicolumn{3}{|c|}{$\begin{array}{l}\text { U.S. Geological Survey } \\
\text { site designation }\end{array}$}} & \multirow{3}{*}{$\begin{array}{c}\text { Depth } \\
\text { of } \\
\text { well } \\
\text { (feet) }\end{array}$} & \multirow{3}{*}{$\begin{array}{c}\text { Land- } \\
\text { surface } \\
\text { altitude } \\
\text { (feet above } \\
\text { sea level) }\end{array}$} & \multicolumn{4}{|c|}{ Water-level measurement } \\
\hline & & & & & \multirow[b]{2}{*}{ Date } & \multirow{2}{*}{$\begin{array}{c}\text { Feet } \\
\text { below } \\
\text { land } \\
\text { surface }\end{array}$} & \multirow[b]{2}{*}{ Status } & \multirow[b]{2}{*}{ Method } \\
\hline & $\begin{array}{c}\text { Local } \\
\text { Identification }\end{array}$ & $\begin{array}{c}\text { Standard } \\
\text { Identification }\end{array}$ & & & & & & \\
\hline \multirow[t]{19}{*}{153} & N22 E54 27CAABI & (Continued) & & & $04-22-69$ & 21.26 & -- & $S$ \\
\hline & & & & & $04-22-70$ & 21.41 & -- & $S$ \\
\hline & & & & & $04-20-71$ & 22.26 & - & $\mathrm{S}$ \\
\hline & & & & & $03-03-72$ & 21.03 & -- & $S$ \\
\hline & & & & & $04-28-73$ & 16.02 & $\ldots$ & $S$ \\
\hline & & & & & $05-13-74$ & 23.50 & .. & $S$ \\
\hline & & & & & $03-14-75$ & 26.23 & - & $S$ \\
\hline & & & & & $03-18-76$ & 25.32 & -- & $S$ \\
\hline & & & & & $03-24-77$ & 29.30 & -- & $S$ \\
\hline & & & & & $04-12-78$ & 32.72 & -- & $S$ \\
\hline & & & & & $03-20-79$ & 34.35 & -- & $\mathrm{S}$ \\
\hline & & & & & $03-19-80$ & 37.92 & -- & $\mathrm{S}$ \\
\hline & & & & & $03-20-81$ & 40.05 & -- & $S$ \\
\hline & & & & & $03-16-82$ & 44.27 & -- & $S$ \\
\hline & & & & & $03-25-85$ & 39.48 & -- & $S$ \\
\hline & & & & & $03-27-87$ & 47.62 & -- & $S$ \\
\hline & & , & & & $03-18-88$ & 50.67 & -- & $S$ \\
\hline & & & & & $04-20-89$ & 53.88 & $S$ & $S$ \\
\hline & & & & & $05-24-90$ & 57.70 & -- & $S$ \\
\hline \multirow[t]{20}{*}{153} & N22 E54 28AACCI & 394542115533001 & 184 & 5,856 & $11-10-58$ & 8.0 & -- & $\mathbf{R}$ \\
\hline & & & & & $|1-00-6|$ & 13.5 & -- & $\mathrm{Z}$ \\
\hline & & & & & $10-16-63$ & 15.07 & -- & $S$ \\
\hline & & & & & $11-00-63$ & 17.6 & - & $\mathrm{Z}$ \\
\hline & & & & & $03-00-66$ & 14.6 & -- & $\mathrm{Z}$ \\
\hline & & & & & $11-00-67$ & 28.7 & -- & $\mathrm{Z}$ \\
\hline & & & & & $11-00-68$ & 24.6 & -- & $\mathrm{Z}$ \\
\hline & & & & & $03-00-69$ & 20.5 &.- & $\mathrm{z}$ \\
\hline & & & & & $11-00-69$ & 21.7 & -- & $\mathrm{Z}$ \\
\hline & & & & & $03-00-74$ & 19.2 & - & $\mathrm{Z}$ \\
\hline & & & & & $11-00-75$ & 25.0 & - & Z \\
\hline & & & & & $03-00-76$ & 21.7 & -- & $\mathrm{Z}$ \\
\hline & & & & & $11-00-76$ & 27.8 & -. & $\mathbf{Z}$ \\
\hline & & & & & $03-00-77$ & 24.4 & - & $\mathrm{Z}$ \\
\hline & & & & & $11-00-77$ & 30.6 & -. & $\mathrm{Z}$ \\
\hline & & & & & $03-00-78$ & 29.7 & - & $\mathrm{Z}$ \\
\hline & & & & & $11-00-78$ & 31.0 & -. & $\mathrm{Z}$ \\
\hline & & & & & $11-00-79$ & 45.0 & - & $\mathrm{z}$ \\
\hline & & & & & $11-00-80$ & 40.0 & - & $\mathrm{Z}$ \\
\hline & & & & & $11-00-81$ & 48.8 & - & $\mathrm{Z}$ \\
\hline \multirow[t]{5}{*}{153} & N22 E54 28DCCC1 & 394507115534101 & 350 & 5,862 & $05-30-61$ & 13 & - & $\cdots$ \\
\hline & & & & & $11-00-63$ & 17.6 & - & $\mathrm{Z}$ \\
\hline & & & & & $04-07-66$ & 14.62 & -- & $\mathrm{Z}$ \\
\hline & & & & & $11-00-67$ & 28.7 & $\cdots$ & $\mathrm{Z}$ \\
\hline & & & & & $04-00-68$ & 20.8 & -- & $\mathrm{Z}$ \\
\hline
\end{tabular}


Appendix. Water-level measurements in selected wells, Diamond Valley, Nevada, 1960-90—Continued

\begin{tabular}{|c|c|c|c|c|c|c|c|c|}
\hline \multirow{2}{*}{\multicolumn{3}{|c|}{$\begin{array}{l}\text { U.S. Geological Survey } \\
\text { site designation }\end{array}$}} & \multirow{3}{*}{$\begin{array}{c}\text { Depth } \\
\text { of } \\
\text { well } \\
\text { (feet) }\end{array}$} & \multirow{3}{*}{$\begin{array}{c}\text { Land- } \\
\text { surface } \\
\text { altitude } \\
\text { (feet above } \\
\text { sea level) }\end{array}$} & \multicolumn{4}{|c|}{ Water-level measurement } \\
\hline & & & & & \multirow[b]{2}{*}{ Date } & \multirow{2}{*}{$\begin{array}{c}\text { Feet } \\
\text { below } \\
\text { land } \\
\text { surface }\end{array}$} & \multirow[b]{2}{*}{ Status } & \multirow[b]{2}{*}{ Method } \\
\hline & $\begin{array}{c}\text { Local } \\
\text { identification }\end{array}$ & $\begin{array}{l}\text { Standard } \\
\text { identification }\end{array}$ & & & & & & \\
\hline \multirow{20}{*}{\multicolumn{2}{|c|}{153 N22 E54 28DCCC1 }} & ontinued & & & $08-27-68$ & 24.9 & -- & $\mathrm{Z}$ \\
\hline & & & & & $04-21-69$ & 19.1 & -- & $\mathrm{Z}$ \\
\hline & & & & & $09-12-69$ & 22.9 & $\ldots$ & $Z$ \\
\hline & & & & & $11-15-71$ & 21.8 & -- & $\mathrm{Z}$ \\
\hline & & & & & $02-24-72$ & 20.8 & -. & Z \\
\hline & & & & & $11-29-73$ & 24.1 & .. & Z \\
\hline & & & & & $03-13-74$ & 25.6 & -- & $\mathrm{Z}$ \\
\hline & & & & & $04-00-75$ & 28.4 & -- & $\mathrm{R}$ \\
\hline & & & & & $11-06-75$ & 25.8 & -- & $Z$ \\
\hline & & & & & $04-00-76$ & 24.9 & -- & $z$ \\
\hline & & & & & $11-08-76$ & 32.5 & .. & $\mathrm{Z}$ \\
\hline & & & & & $03-05-77$ & 28.8 & -- & $Z$ \\
\hline & & & & & $11-17-77$ & 35.3 & -- & $Z$ \\
\hline & & & & & 04-04-78 & 33.6 & -. & $Z$ \\
\hline & & & & & $11-14-78$ & 37.5 & -. & $z$ \\
\hline & & & & & $11-07-79$ & 44.7 & -- & $z$ \\
\hline & & & & & $04-17-80$ & 40.5 & -. & $\mathrm{Z}$ \\
\hline & & & & & $11-21-80$ & 46.3 & -- & $\mathrm{Z}$ \\
\hline & & & & & $11-09-82$ & 48.9 & -. & $\mathrm{Z}$ \\
\hline & & & & & $04-01-83$ & 45.3 & -- & $\mathrm{Z}$ \\
\hline \multirow[t]{12}{*}{153} & $\mathrm{~N} 22 \mathrm{E} 54$ 32BCCCl & 394439115552901 & 259 & 5,864 & $11-03-75$ & 30.5 & -- & $z$ \\
\hline & & & & & $03-05-77$ & 32.0 & -- & $\mathrm{Z}$ \\
\hline & & & & & $11-14-77$ & 43.3 & -- & $\mathrm{Z}$ \\
\hline & & & & & $04-04-78$ & 36.2 & -- & Z \\
\hline & & & & & 11-08-78 & 46.6 & -. & Z \\
\hline & & & & & $11-01-79$ & 51.6 & -- & Z \\
\hline & & & & & $04-17-80$ & 45.7 & .- & Z \\
\hline & & & & & $11-01-80$ & 48.5 & -- & Z \\
\hline & & & & & $11-01-81$ & 55.2 & -. & $\bar{Z}$ \\
\hline & & & & & $11-09-82$ & 55.3 & -- & 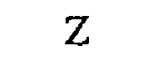 \\
\hline & & & & & $05-01-83$ & 53.4 & -- & $Z$ \\
\hline & & & & & $11-02-89$ & 71.6 & -. & $z$ \\
\hline \multirow[t]{10}{*}{$153 \mathrm{I}$} & N22 E54 33BBDD1 & 394452115540801 & 300 & 5,865 & $05-01-61$ & 60 & -- & -. \\
\hline & & & & & $11-00-64$ & 18.4 & -- & $\mathrm{R}$ \\
\hline & & & & & $04-07-66$ & 15.68 & -- & $\mathrm{Z}$ \\
\hline & & & & & $11-00-66$ & 18.3 & -- & $\mathrm{R}$ \\
\hline & & & & & $11-00-67$ & 26.0 & -- & $\mathrm{R}$ \\
\hline & & & & & $04-00-68$ & 21.5 & -- & $\mathrm{R}$ \\
\hline & & & & & $08-27-68$ & 27.0 & -- & Z \\
\hline & & & & & $04-21-69$ & 21.4 & .. & $\mathrm{Z}$ \\
\hline & & & & & $09-12-69$ & 25.4 & -- & $\mathrm{Z}$ \\
\hline & & & & & $11-16-71$ & 24.3 & -- & Z \\
\hline
\end{tabular}


Appendix. Water-level measurements in selected wells, Diamond Valley, Nevada, 1960-90—Continued

\begin{tabular}{|c|c|c|c|c|c|c|c|c|}
\hline \multirow{2}{*}{\multicolumn{3}{|c|}{$\begin{array}{l}\text { U.S. Geological Survey } \\
\text { site designation }\end{array}$}} & \multirow{3}{*}{$\begin{array}{c}\text { Depth } \\
\text { of } \\
\text { well } \\
\text { (feet) }\end{array}$} & \multirow{3}{*}{$\begin{array}{l}\text { Land- } \\
\text { surface } \\
\text { altitude } \\
\text { (feet above } \\
\text { sea level) }\end{array}$} & \multicolumn{4}{|c|}{ Water-level measurement } \\
\hline & & & & & \multirow[b]{2}{*}{ Date } & \multirow{2}{*}{$\begin{array}{c}\text { Feet } \\
\text { below } \\
\text { land } \\
\text { surface }\end{array}$} & \multirow[b]{2}{*}{ Status } & \multirow[b]{2}{*}{ Method } \\
\hline & $\begin{array}{c}\text { Local } \\
\text { identification }\end{array}$ & $\begin{array}{c}\text { Standard } \\
\text { identification }\end{array}$ & & & & & & \\
\hline \multirow{15}{*}{\multicolumn{3}{|c|}{153 N22 E54 33BBDD1 (Continued) }} & & & $08-10-73$ & 37.1 & -- & $\mathrm{Z}$ \\
\hline & & & & & $11-29-73$ & 23.2 & -- & $\mathrm{Z}$ \\
\hline & & & & & $03-13-74$ & 23.6 & -. & Z \\
\hline & & & & & $11-06-75$ & 30.5 & -- & $\mathrm{Z}$ \\
\hline & & & & & $04-07-76$ & 31.2 & $\cdots$ & $\mathrm{Z}$ \\
\hline & & & & & $11-08-76$ & 34.7 & -- & $\mathrm{Z}$ \\
\hline & & & & & $03-05-77$ & 30.8 & -- & $\mathrm{Z}$ \\
\hline & & & & & $11-17-77$ & 37.6 & -- & $\mathrm{Z}$ \\
\hline & & & & & $04-04-78$ & 33.5 & -- & $\mathrm{Z}$ \\
\hline & & & & & $11-14-78$ & 35.5 & -- & $\mathrm{Z}$ \\
\hline & & & & & $11-07-79$ & 46.1 & -- & $\mathrm{Z}$ \\
\hline & & & & & $04-17-80$ & 39.5 & -- & $\mathbf{Z}$ \\
\hline & & & & & $11-21-80$ & 45.2 & $\cdots$ & $\mathrm{Z}$ \\
\hline & & & & & $11-09-82$ & 57.9 & - & $\mathrm{Z}$ \\
\hline & & & & & $04-01-83$ & 55.2 & -- & $\mathrm{Z}$ \\
\hline \multirow{25}{*}{\multicolumn{2}{|c|}{ I53 N23 E53 27BB 1}} & 395100115593001 & 22 & 5,820 & $09-16-64$ & 12.64 & -- & $S$ \\
\hline & & & & & $04-23-65$ & 12.59 & -- & $S$ \\
\hline & & & & & $10-21-65$ & 12.63 & -- & $S$ \\
\hline & & & & & $04-08-66$ & 12.57 & -- & $S$ \\
\hline & & & & & $11-18-66$ & 12.52 & $\mathrm{Z}$ & $\mathrm{S}$ \\
\hline & & & & & $03-20-68$ & 12.65 & -- & $S$ \\
\hline & & & & & $04-22-69$ & 11.60 & -- & $S$ \\
\hline & & & & & $04-22-70$ & 12.62 & -- & $\mathrm{S}$ \\
\hline & & & & & $04-20-71$ & 12.63 & -- & $\mathrm{S}$ \\
\hline & & & & & $03-03-72$ & 12.73 & -- & $S$ \\
\hline & & & & & $04-28-73$ & 12.76 & -- & $S$ \\
\hline & & & & & $05-13-74$ & 12.85 & -- & $\mathrm{S}$ \\
\hline & & & & & $03-13-75$ & 12.89 & -- & $S$ \\
\hline & & & & & $03-18-76$ & 12.88 & -- & -- \\
\hline & & & & & $03-24-77$ & 12.97 & -- & $S$ \\
\hline & & & & & $04-12-78$ & 13.04 & -- & $S$ \\
\hline & & & & & $03-20-79$ & 13.09 & -- & $S$ \\
\hline & & & & & $03-19-80$ & 13.21 & -- & $S$ \\
\hline & & & & & $03-20-81$ & 13.27 & -- & $S$ \\
\hline & & & & & $03-16-82$ & 13.36 & -- & $\mathrm{S}$ \\
\hline & & & & & $03-25-85$ & 13.03 & -- & $S$ \\
\hline & & & & & $02-09-87$ & 13.34 & -- & $S$ \\
\hline & & & & & $03-18-88$ & 13.46 & -- & $\mathrm{S}$ \\
\hline & & & & & $04-20-89$ & 13.55 & -- & $S$ \\
\hline & & & & & $05-24-90$ & 13.34 & -- & $S$ \\
\hline \multirow{5}{*}{\multicolumn{2}{|c|}{153 N23 E53 30DD 1}} & 395020116030001 & 22 & 5,821 & $09-16-64$ & 14.21 & -- & $S$ \\
\hline & & & & & $04-23-65$ & 14.15 & -- & $S$ \\
\hline & & & & & $10-21-65$ & 14.17 & -- & $S$ \\
\hline & & & & & $04-08-66$ & 14.12 & -- & $S$ \\
\hline & & & & & $11-18-66$ & 14.24 & $\mathrm{Z}$ & $S$ \\
\hline
\end{tabular}


Appendix. Water-level measurements in selected wells, Diamond Valley, Nevada, 1960-90-Continued

\begin{tabular}{|c|c|c|c|c|c|c|c|c|}
\hline \multirow{2}{*}{\multicolumn{3}{|c|}{$\begin{array}{l}\text { U.S. Geological Survey } \\
\text { site designation }\end{array}$}} & \multirow{3}{*}{$\begin{array}{c}\text { Depth } \\
\text { of } \\
\text { well } \\
\text { (feet) }\end{array}$} & \multirow{3}{*}{$\begin{array}{c}\text { Land- } \\
\text { surface } \\
\text { altitude } \\
\text { (feet above } \\
\text { sea level) }\end{array}$} & \multicolumn{4}{|c|}{ Water-level measurement } \\
\hline & & & & & \multirow[b]{2}{*}{ Date } & \multirow{2}{*}{$\begin{array}{c}\text { Feet } \\
\text { below } \\
\text { land } \\
\text { surface }\end{array}$} & \multirow[b]{2}{*}{ Status } & \multirow[b]{2}{*}{ Method } \\
\hline & $\begin{array}{c}\text { Local } \\
\text { identification }\end{array}$ & $\begin{array}{c}\text { Standard } \\
\text { identification }\end{array}$ & & & & & & \\
\hline \multirow[t]{20}{*}{153} & N23 E53 30DD & I (Continued) & & & $03-20-68$ & 14.15 & -- & $S$ \\
\hline & & & & & $04-22-69$ & 16.16 & -- & $\mathrm{S}$ \\
\hline & & & & & $04-22-70$ & 14.05 & -- & $S$ \\
\hline & & & & & $04-20-71$ & 14.07 & -- & $\mathrm{S}$ \\
\hline & & & & & $03-03-72$ & 14.15 & -- & $S$ \\
\hline & & & & & $04-28-73$ & 14.08 & -- & $S$ \\
\hline & & & & & $05-13-74$ & 14.26 & -- & $S$ \\
\hline & & & & & $03-13-75$ & 14.28 & -- & $S$ \\
\hline & & & & & $03-18-76$ & 14.27 & -- & -- \\
\hline & & & & & $03-24-77$ & 14.39 & -- & $\mathbf{S}$ \\
\hline & & & & & $04-12-78$ & 14.50 & $\cdots$ & $S$ \\
\hline & & & & & $03-20-79$ & 14.50 & -- & $\mathrm{S}$ \\
\hline & & & & & $03-19-80$ & 14.68 & -- & $\mathrm{S}$ \\
\hline & & & & & $03-20-81$ & 14.66 & - & $\mathrm{S}$ \\
\hline & & & & & $03-16-82$ & 14.72 & -- & $\mathbf{S}$ \\
\hline & & & & & $03-25-85$ & 14.14 & -- & $\mathrm{S}$ \\
\hline & & & & & $02-09-87$ & 14.44 & -- & $\mathrm{S}$ \\
\hline & & & & & $03-18-88$ & 14.62 & -- & $S$ \\
\hline & & & & & $04-20-89$ & 14.76 & -- & $S$ \\
\hline & & & & & $05-24-90$ & 14.42 & -- & $\mathrm{S}$ \\
\hline \multirow[t]{24}{*}{153} & N23 E54 18DB & 395220115561001 & 32 & 5,800 & $09-16-64$ & 17.26 & -- & $\mathrm{S}$ \\
\hline & & & & & $04-23-65$ & 17.01 & -- & $S$ \\
\hline & & & & & $10-21-65$ & 17.21 & -- & $S$ \\
\hline & & & & & $04-08-66$ & 16.64 & -- & $\mathrm{S}$ \\
\hline & & & & & $11-18-66$ & 16.45 & $\mathbf{Z}$ & $S$ \\
\hline & & & & & $03-20-68$ & 16.97 & -- & $S$ \\
\hline & & & & & $04-22-69$ & 16.93 & -- & $\mathrm{S}$ \\
\hline & & & & & $04-22-70$ & 16.78 & -- & $S$ \\
\hline & & & & & $04-20-71$ & 16.78 & -- & $\mathrm{S}$ \\
\hline & & & & & $03-03-72$ & 16.89 & -- & $S$ \\
\hline & & & & & $04-28-73$ & 16.74 & -- & $\mathrm{S}$ \\
\hline & & & & & $05-13-74$ & 16.85 & -- & $S$ \\
\hline & & & & & $03-13-75$ & 17.10 & -- & $\mathbf{S}$ \\
\hline & & & & & $03-18-76$ & 17.15 & -- & -- \\
\hline & & & & & $04-12-78$ & 17.45 & -- & $\mathrm{S}$ \\
\hline & & & & & $03-20-79$ & 17.65 & -- & $\mathrm{S}$ \\
\hline & & & & & $03-19-80$ & 17.91 &.- & $S$ \\
\hline & & & & & $03-20-81$ & 17.97 & -- & $\mathrm{S}$ \\
\hline & & & & & $03-16-82$ & 18.24 & -- & $S$ \\
\hline & & & & & $03-25-85$ & 18.02 & -- & $\mathrm{S}$ \\
\hline & & & & & $02-09-87$ & 18.10 & -- & $S$ \\
\hline & & & & & $03-18-88$ & 18.08 & -- & $S$ \\
\hline & & & & & $04-20-89$ & 17.98 & -- & $\mathrm{S}$ \\
\hline & & & & & $05-24-90$ & 17.55 & -- & $S$ \\
\hline
\end{tabular}


Appendix. Water-level measurements in selected wells, Diamond Valley, Nevada, 1960-90-Continued

\begin{tabular}{|c|c|c|c|c|c|c|c|c|}
\hline \multirow{2}{*}{\multicolumn{3}{|c|}{$\begin{array}{l}\text { U.S. Geological Survey } \\
\text { site designation }\end{array}$}} & \multirow{3}{*}{$\begin{array}{c}\text { Depth } \\
\text { of } \\
\text { well } \\
\text { (feet) }\end{array}$} & \multirow{3}{*}{$\begin{array}{l}\text { Land- } \\
\text { surface } \\
\text { altitude } \\
\text { (feet above } \\
\text { sea level) }\end{array}$} & \multicolumn{4}{|c|}{ Water-level measurement } \\
\hline & & & & & \multirow[b]{2}{*}{ Date } & \multirow{2}{*}{$\begin{array}{c}\text { Feet } \\
\text { below } \\
\text { land } \\
\text { surface }\end{array}$} & \multirow[b]{2}{*}{ Status } & \multirow[b]{2}{*}{ Method } \\
\hline & $\begin{array}{l}\text { Local } \\
\text { identification }\end{array}$ & $\begin{array}{l}\text { Standard } \\
\text { identification }\end{array}$ & & & & & & \\
\hline \multirow[t]{26}{*}{153} & N23 E54 29CDDD1 & 395019115543801 & 189 & 5,827 & $11-00-64$ & 5.8 & -- & $\mathrm{z}$ \\
\hline & & & & & $11-00-65$ & 3.8 & -- & $\mathrm{Z}$ \\
\hline & & & & & $03-00-66$ & 1.6 & -- & $\mathrm{Z}$ \\
\hline & & & & & $04-07-66$ & 1.62 & - & $\mathrm{S}$ \\
\hline & & & & & $11-00-66$ & 4.8 & -- & $\mathrm{Z}$ \\
\hline & & & & & $03-00-67$ & 3.8 & -- & $\mathrm{Z}$ \\
\hline & & & & & $11-00-67$ & 7.7 & - & $\mathrm{Z}$ \\
\hline & & & & & $03-00-68$ & 4.4 & -- & $\mathrm{Z}$ \\
\hline & & & & & $11-00-68$ & 9.8 & -- & $\mathrm{z}$ \\
\hline & & & & & $11-00-69$ & 7.1 & -- & $\mathrm{Z}$ \\
\hline & & & & & $|1-00-7|$ & 5.7 & -- & $\mathrm{z}$ \\
\hline & & & & & $03-00-77$ & 8.6 & -. & $\mathrm{Z}$ \\
\hline & & & & & $11-14-77$ & 16.5 & -- & $\mathrm{Z}$ \\
\hline & & & & & $11-08-78$ & 15.2 & -- & $\mathrm{z}$ \\
\hline & & & & & $11-01-79$ & 23.4 & -- & $\mathrm{Z}$ \\
\hline & & & & & $11-01-80$ & 24.1 & -- & $\mathrm{Z}$ \\
\hline & & & & & $11-01-81$ & 31.4 & -- & $\mathrm{Z}$ \\
\hline & & & & & $11.09-82$ & 25.5 & -- & $\mathrm{z}$ \\
\hline & & & & & $05-01-83$ & 16.7 & -- & $\mathrm{z}$ \\
\hline & & & & & $11-07-83$ & 26.4 & - & $\mathrm{z}$ \\
\hline & & & & & $11-01-84$ & 28.8 & .- & $\mathrm{z}$ \\
\hline & & & & & $12-03-85$ & 25.6 & -- & $\mathrm{z}$ \\
\hline & & & & & $11-04-86$ & 32.3 & -- & $\mathrm{Z}$ \\
\hline & & & & & $11-12-87$ & 33.4 & -- & $\mathrm{Z}$ \\
\hline & & & & & $10-17-88$ & 41.5 & -- & $\mathrm{Z}$ \\
\hline & & & & & $11-02-89$ & 42.8 & -- & $\mathrm{z}$ \\
\hline \multirow[t]{15}{*}{153} & N23 E54 30DDD 1 & 395019115551501 & 220 & 5,829 & $07-19-65$ & 4.0 & -- & -- \\
\hline & & & & & $04-07-66$ & 2.31 & -- & $\mathrm{R}$ \\
\hline & & & & & $08-28-68$ & 7.0 & -- & $\mathrm{Z}$ \\
\hline & & & & & $09-17-69$ & 5.4 & -- & $\mathrm{z}$ \\
\hline & & & & & $11-16-71$ & 2.5 & -- & $\mathrm{z}$ \\
\hline & & & & & $08-10-73$ & 3.8 & -- & $\mathrm{Z}$ \\
\hline & & & & & $03-05-77$ & 6.3 & -- & $\mathrm{Z}$ \\
\hline & & & & & $11-17-77$ & 13.2 & -- & $\mathrm{Z}$ \\
\hline & & & & & $04-07-78$ & 9.7 & -- & $\mathrm{Z}$ \\
\hline & & & & & $11-14-78$ & 14.8 & -- & $z$ \\
\hline & & & & & $11-07-79$ & 21.0 & -- & $\mathrm{Z}$ \\
\hline & & & & & $04-17-80$ & 14.2 & -- & $\mathrm{Z}$ \\
\hline & & & & & $12-01-80$ & 19.3 & -- & $\mathrm{z}$ \\
\hline & & & & & $11-09-82$ & 23.0 & -- & $\mathrm{Z}$ \\
\hline & & & & & $04-01-83$ & 8.8 & -- & $\mathrm{z}$ \\
\hline \multirow[t]{5}{*}{153} & N23 E54 32DCCCl & 394927115543601 & 280 & 5,831 & $06-05-64$ & 15 & -- & $\mathrm{R}$ \\
\hline & & & & & $04-00-66$ & 2.21 & -- & $\mathrm{Z}$ \\
\hline & & & & & $11-00-66$ & 7.0 & -- & $\mathrm{z}$ \\
\hline & & & & & $04-00-68$ & 5.1 & -- & $\mathrm{z}$ \\
\hline & & & & & $08-28-68$ & 13.0 & -- & $\mathrm{Z}$ \\
\hline
\end{tabular}


Appendix. Water-level measurements in selected wells, Diamond Valley, Nevada, 1960-90-Continued

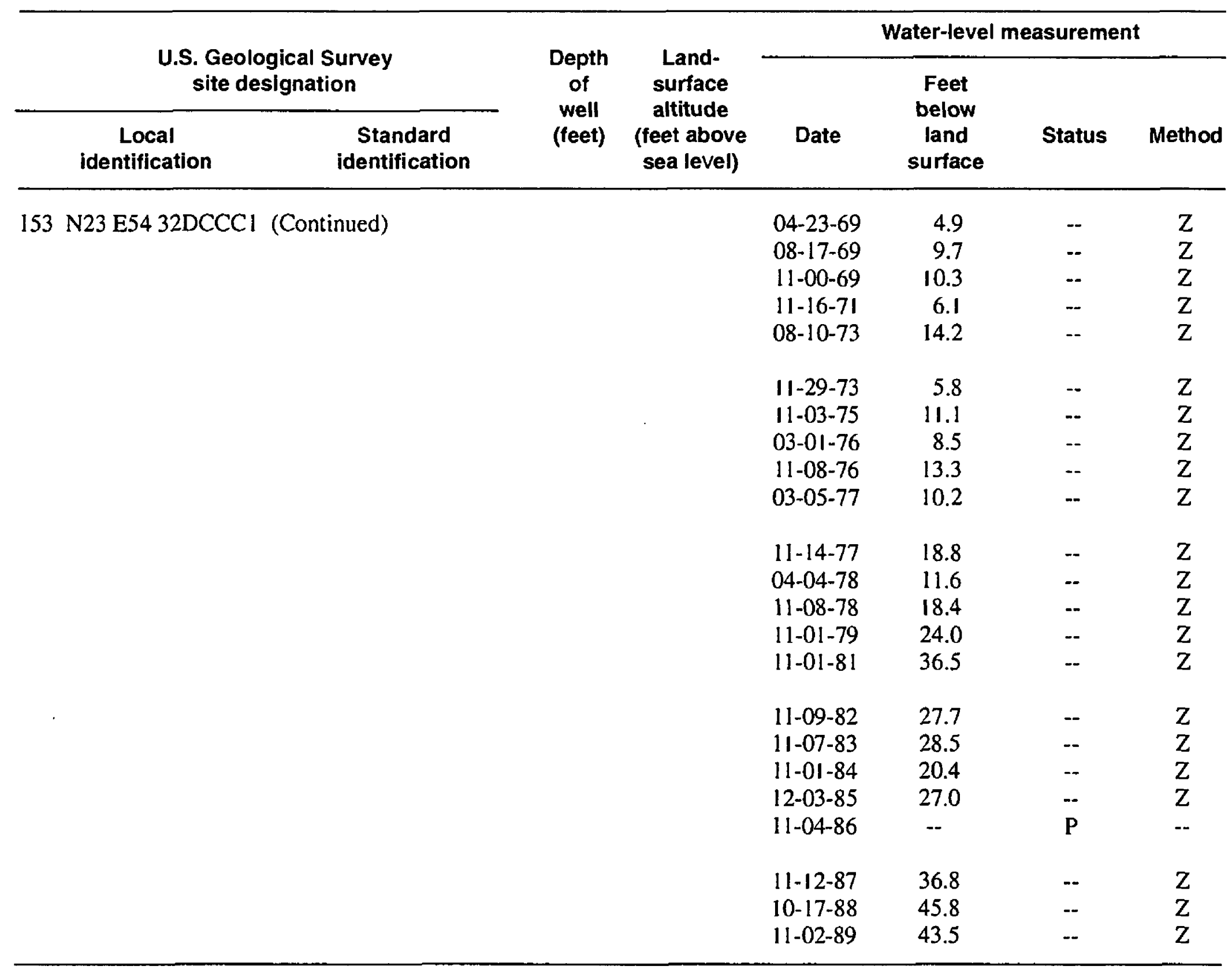

